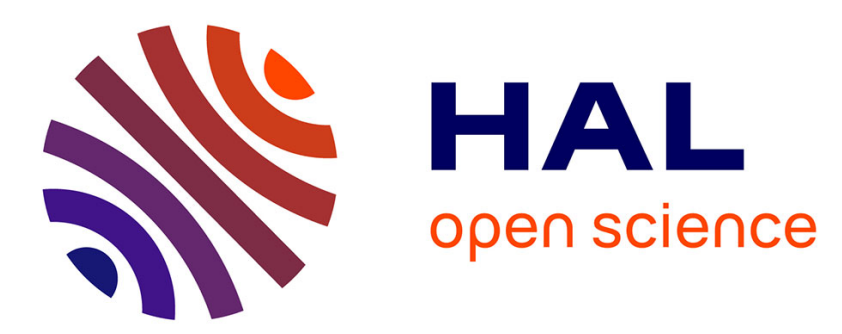

\title{
The grid method for in-plane displacement and strain measurement: a review and analysis
}

Michel Grediac, Frédéric Sur, Benoît Blaysat

\section{To cite this version:}

Michel Grediac, Frédéric Sur, Benoît Blaysat. The grid method for in-plane displacement and strain measurement: a review and analysis. Strain, 2016, 52 (3), pp.205-243. 10.1111/str.12182 . hal01317145

\section{HAL Id: hal-01317145 \\ https://inria.hal.science/hal-01317145}

Submitted on 2 Jun 2016

HAL is a multi-disciplinary open access archive for the deposit and dissemination of scientific research documents, whether they are published or not. The documents may come from teaching and research institutions in France or abroad, or from public or private research centers.
L'archive ouverte pluridisciplinaire HAL, est destinée au dépôt et à la diffusion de documents scientifiques de niveau recherche, publiés ou non, émanant des établissements d'enseignement et de recherche français ou étrangers, des laboratoires publics ou privés. 


\title{
The grid method for in-plane displacement and strain measurement: a review and analysis
}

\author{
Michel GREDIAC ${ }^{1 \dagger}$, Frédéric SUR ${ }^{2}$, Benoît BLAYSAT ${ }^{1}$ \\ ${ }^{1}$ Clermont Université, Université Blaise Pascal, Institut Pascal, UMR CNRS 6602 \\ BP 10448, 63000 Clermont-Ferrand, France \\ ${ }^{2}$ Laboratoire Lorrain de Recherche en Informatique et ses Applications, UMR CNRS 7503 \\ Université de Lorraine, CNRS, INRIA projet Magrit, Campus Scientifique, BP 239, 54506 \\ Vandoeuvre-lès-Nancy Cedex, FRANCE \\ $\dagger$ corresponding author, tel: +3347328 80 77, fax: +3347328 80 27, \\ michel.grediac@univ-bpclermont.fr
}

\begin{abstract}
The grid method is a technique suitable for the measurement of in-plane displacement and strain components on specimens undergoing a small deformation. It relies on a regular marking of the surfaces under investigation. Various techniques are proposed in the literature to retrieve these sought quantities from images of regular markings, but recent advances show that techniques developed initially to process fringe patterns lead to the best results. The grid method features a good compromise between measurement resolution and spatial resolution, thus making it an efficient tool to characterize strain gradients. Another advantage of this technique is the ability to establish closed-form expressions between its main metrological characteristics, thus enabling to predict them within certain limits. In this context, the objective of this paper is to give the state of the art in the grid method, the information being currently spread out in the literature. We propose first to recall various techniques which were used in the past to process grid images, to focus progressively on the one which is the most used in recent examples: the windowed Fourier transform. From a practical point of view, surfaces under investigation must be marked with grids, so the techniques available to mark specimens with grids are presented. Then we gather the information available in the recent literature to synthesise the connection between three important characteristics of full-field measurement techniques: the spatial resolution, the measurement resolution, and the measurement bias. Some practical information is then offered to help the readers who discover this technique to start using it. In particular, programmes used here to process the grid images are offered to the readers on a dedicated website. We finally present some recent examples available in the literature to highlight the effectiveness of the grid method for in-plane displacement and strain measurement in real situations.
\end{abstract}

Keywords: displacement, full-field measurement, grid method, strain.

This is the author-manuscript version of

M. Grédiac, F. Sur, and B. Blaysat. The grid method for in-plane displacement and strain measurement: a review and analysis. Strain, vol. 52, no. 3, p. 205243, Wiley, 2016.

DOI: $10.1111 /$ str. 12182 


\section{Introduction}

Full-field measurement techniques are now widespread in the experimental mechanics community [1, 2]. This is mainly due to the combined effect of the decreasing cost of cameras, their increasing performance, and the very nature of the quantities they provide: fields of measurements such as displacements or strains. Indeed this wealth of data can be used in different ways for a better characterisation of materials and structures. It offers for instance the possibility to validate the output of numerical models obtained with finite element calculations, to observe phenomena whose location cannot be predicted in advance, to help propose suitable constitutive models able to describe and predict these observed phenomena, and even to identify their governing parameters. Some techniques based on incoherent light such as moiré [3, 4, 5, 6] have been known for a long time but they require the use of two gratings: a reference and a deformed one. Others such as electronic speckle interferometry [7], holographic interferometry [8] and moiré interferometry [9] rely on coherent light, so they are somewhat complicated to implement in experimental mechanics laboratories. Because of their ease of use, white-light techniques have become more popular in experimental mechanics. In particular, digital image correlation [10] is now widely accepted, as reflected in the huge body of literature available on this technique or on its use in experimental mechanics, see for instance the review proposed in [11. Beside this well-known technique based on the processing of randomly marked surfaces, a white-light technique based on the processing of regular patterns, namely the grid method, is also employed. This latter technique combines three main advantages:

- Like DIC, this is a non-interferometric technique;

- It can potentially take benefit of some efficient and well-established procedures developed for processing fringes obtained with interferometric techniques;

- It relies on reproducible patterns.

This last feature can be an asset for future standardization of full-field measurement techniques. The main drawback for the grid method is that the surface must be marked with a pattern, which must be as regular as possible. However, even optimised random patterns have been defined for DIC [12, thus depositing a controlled pattern is also an issue with DIC.

In this context, the main objective of this paper is to focus on the technical characteristics and the metrological performance of this contactless optical method. The paper is organized as follows. Regularly marked surfaces have been employed for a long time to measure displacement and strain components, but processing images of these regular markings can potentially be made with different approaches which are briefly presented in Section 2. We discuss then the different techniques available to mark surfaces with grids in practice. This is the aim of Section 3 . Section 4 is a reminder on the windowed Fourier transform (WFT), which is the most commonly technique used to process grid images. Providing the metrological performance is necessary for any measuring tool, so Section 5 discusses in depth the performances and limits of the grid method, with a particular emphasis put on three metrological characteristics which are first defined, namely the measurement resolution, the bias and the spatial resolution. Then we discuss in Section 6 some practical problems raised when using this technique, before finally presenting in the last section some recent applications of the grid method for material characterisation purposes. 


\section{The grid method: what are we talking about?}

We address here the measurement of in-plane displacement and strain components by processing images of flat specimens on which a regular marking such as a grid has been deposited before in-plane deformation. The idea is quite old since it flows from intuition: considering a regular marking enables us to easily distinguish features such as particular points on deformed marked surfaces, and thus facilitates tracking them during deformation. The use of grids to build regular distributions of points by considering either the intersection of the lines or the dot points located in between is broadly documented in the literature of the 40's-60's, as reported in a survey paper published in the late 60's [13. Isolated but regularly distributed points were also employed in the same spirit [14]. At that time, pictures of deformed grids were processed by hand, by measuring the coordinates of the points, and deducing the displacement by subtracting the coordinates between current and reference configurations. Even though the progressive dissemination of affordable digital cameras and powerful computational resources together with recent advances in image processing led finding the coordinates of the points automatically and more precisely than by hand [15], this technique was mainly used for measuring large displacements and strains like those occurring in composite or metal sheet forming [16, 17, 18, 19, 20, 21]. It is now only scarcely mentioned in recent papers such as $22,23,24$, and seems to fall progressively into disuse. The main cause is the emergence and dissemination of digital image correlation (DIC) in the experimental mechanics community [25, 10]. DIC can potentially process images of regular markings [26] but this technique mainly relies on randomly marked surfaces, which undoubtedly constitutes an advantage from a practical point of view. This has led this technique to be now widely accepted and employed in experimental mechanics, as illustrated in [27]. Despite the versatility and the user-friendliness of DIC, this technique suffers from some disadvantages pointed out by experts in the field, for instance in case of non-homogeneous and small deformation [11. On the contrary, interferometric techniques exhibit a better measurement resolution, but they require the use of various optical components such as mirrors, lasers or beam splitters, which make them difficult to use in an industrial context. In addition, these techniques are often highly sensitive to vibrations. Trying to overcome the disadvantages of DIC without relying on interferometry has led some researchers to still consider regular grids instead of random patterns, this time not by tracking the intersecting points between the lines or the dot points located in between, but by employing numerical tools developed for a similar objective: namely processing fringes by extracting phase distributions from regular patterns.

It is clear that from a practical point of view, depositing regular patterns may appear incongruous since this induces an additional problem compared to merely spraying a random marking and applying DIC. This is all the truer as grids featuring a frequency of some lines per millimeter are the most suitable ones for thoroughly analyzing phenomena which commonly occur at the scale of usual tensile specimens for instance. However such grids are presently not easily commercially available. This leads users to order specifically printed grids, and then transfer them on the specimens to be tested, see [28] for instance, which does not facilitate the dissemination this technique. Despite this relative drawback, this technique has a big advantage: the possibility to reveal localized phenomena thanks to its good compromise between spatial resolution and measurement resolution, as illustrated in the examples shown in Section 7, which makes it an appealing tool.

As a last remark, it is worth mentioning that the grid method is basically suitable for in-plane measurements, but it has been recently shown that it could be extended to the simultaneous measurement of out-of-plane displacement [29]. 
The grid method would not exist without techniques able to deposit grids on the surface of specimens, so let us now examine the procedures which can be employed to deposit grids on the surface of flat specimens.

\section{Marking surfaces with grids}

As recalled above, grids have been deposited on flat specimens for a long time. In [13, 30, 31, comprehensive lists of techniques available for obtaining grids were given some decades ago. Some of these techniques are still valid with current printing techniques, but the pitches reached and the quality of the obtained grids are generally not available. We thus offer below a state of the art on the techniques employed to mark specimens with grids, by going from the finest to the largest scales.

The finest grid that has been considered for in-plane deformation measurement seems to be the atomic lattice of the substrate itself observed with high resolution electron microscopes (HREM), see Ref. [32, 33, 34], or atomic force microscopes (AFM) [35, 36, 37]. With such natural markings, the grid pitch can be as small as some angströms.

At a higher scale, pitches of some tenths of $\mu \mathrm{m}$ to some $\mu \mathrm{m}$ can be obtained by various techniques such as nanoimprint lithography [38. With the so-called electron beam moiré technique, the lines of the reference grid are "drawn" on the substrate by using the electron beam of a scanning electron microscope (SEM) 39, 40, 41, 42, or milled using a focused ion beam [43, 44, 45, 46. Microgrids can also be obtained by metal sputtering and evaporation [26, 47, 48] or by appropriate etching of the specimen [49]. A simple technique also consists in replicating the negative imprint of a mold grating, see [50, 51, 52, 53, 54 for instance. In this case, the surface of the specimen is polished, cleaned and gel-coated with a suitable epoxy resin beforehand. Optical techniques can also be employed. For instance, photoresist coatings insulated through a mask lead to grid featuring similar pitch values as in the preceding examples [55, 56]. An interferometric setup can also be used to generate a regular marking, as described in [57].

Even at this scale, grids can be used to form moiré fringes from which in-plane displacement fields are deduced. The reference grid is typically the SEM monitor [58] in this case, or a fringe pattern generated by a computer [37, while the deformed grid is that printed on the specimen. Another technique is based on such microgrids: moiré interferometry. In this case, the grid deposited on the specimen acts as a diffraction grating and interference fringes are obtained with two coherent laser beams [9, 59]. Microgrids can also be used to improve the contrast of the natural surface marking, the images being then processed by tracking the dot points [26] or by using digital image correlation [60. Microgrids can also be considered as regular patterns whose phases are modulated by the displacement, thus justifying to employ techniques based on Fourier analysis to retrieve the displacement and strain fields [32, 33, 34, 57, 61] for instance.

Grid frequencies lying between 250 and 2000 lines per inch (thus pitches lying between 102 and $13 \mu \mathrm{m}$, respectively) are reported in 62 by evaporation of aluminium through a mask. In a more recent work, similar grids were obtained by depositing a thin layer of metallic film onto the specimen surface, which was covered beforehand with a copper gilder grid acting as a mask. A very thin layer of gold was then sputter-coated onto the specimen surface. The zone underneath the mesh bars was not coated contrary to the space inside each grid, thus generating regular arrays of spots. Pitches equal to $12.5 \mu \mathrm{m}$ are reported in [22], but other pitch values can potentially be obtained with the same technique: from 12.5 to $500 \mu \mathrm{m}$ in 63 .

In the preceding cases, the imaging system which captures the grid images is often 
behind a microscope because of the very small pitch size, explaining that all the corresponding applications deal with micromechanics. In experimental mechanics however, most of the tests are performed with conventional tensile machines on specimens which are much bigger in size. In addition, for the sake of simplicity, experimentalists generally wish to employ techniques based on white-light illumination, without using any other imaging system than common camera lenses. Taking into account the usual size of the specimens and the number of pixels of current digital camera sensors, grids must feature pitches greater than in the preceding cases when no microscope is used, typically some hundreds of micrometers (often between 100 and $200 \mu \mathrm{m}$ ). Various techniques have been proposed in the literature for this range of pitches. In 64 for instance, grids with a pitch equal to $76.3 \mu \mathrm{m}$ were obtained by stencilling and painting. Even though such grids could be processed by Fourier analysis, it seems that only rather large strains could be measured because of the low quality of the resulting grids, in particular in terms of grid pitch regularity. In [65, it is proposed to evaporate aluminium through a mask grid to mark a polymeric specimen with a grid featuring a pitch equal to $125 \mu \mathrm{m}$. The main drawback here is the fact that a vacuum chamber is needed. In [28], grids with a minimum pitch equal to $100 \mu \mathrm{m}$ were obtained by first printing the grid on a soft polymeric substrate, and then transferring the black ink only onto the tested specimen using a thin layer of white adhesive. High-resolution photoplotters must be used to insure the lowest defects as possible in the printed grids. The lithography technique described above for microgrids can also be employed for higher scales, as reported in [66], where the pitch was equal to $400 \mu \mathrm{m}$. Grids can also be printed directly on decal paper: in [67], grids with a minimum pitch equal to $250 \mu \mathrm{m}$ were printed on this type of paper, and then deposited on the tested specimen. Such a support is cheap and commercially available, see 68 for instance. A drawback is that the thickness of the layer which is deposited is generally not negligible, and its strain at failure is much higher than that of current materials used in mechanical engineering. Furthermore, its adhesion property is reported to be limited under straining [67]. This leads this solution not to be recommended in the case of crack for instance, the layer potentially bridging the crack and the apparent strain near the crack tip being also potentially influenced by the elasticity of the layer. Finally, a transfer printing technique is described in [69, 70] with grids supplied by Mecanorma. The pitch was equal to $0.575 \mathrm{~mm}$ in this case.

Grids with greater pitches are easier to obtain than the aforementioned ones. Paint sprayed through a stencil permitted to obtain pitches equal to $800 \mu \mathrm{m}$ in [71] and $1 \mathrm{~mm}$ in [72, respectively. Pitches equal to $1 \mathrm{~mm}$ are even reported to be drawn by a black pen held in a computerized milling machine in [73. Grids with a pitch equal to $1 \mathrm{~mm}$ can also be obtained with the transfer printing technique, as reported in [69]. Beyond $1 \mathrm{~mm}$ in pitch, stencilling still works since a pitch equal to $2.5 \mathrm{~mm}$ is reported in [66]. Grids can also be photograved on an adhesive tape which is then bonded on the specimen, as in [74] where the grid pitch is equal to $2 \mathrm{~mm}$. As already mentioned in the case of decal paper, such a solution is however not adapted in case of cracks. In large-scale structures, grids can be painted directly onto the surface, as in [75, 76] where pitches equal to $38.5 \mathrm{~mm}$ were obtained with this simple technique.

All the techniques reported here are generally described for marking flat specimens, but it is worth mentioning that regular grids can also be deposited directly on curved ones [77].

As a final remark, it is important to note that the regularity of the pattern is not critical when tracking either the dot points located between the grid lines, or the intersecting points between the lines. This is not the case for spectral approaches relying on the phase 
change between current and reference images, a grid image being modeled as a 2D phasemodulated signal. Possible defects such as spatial fluctuations of the grid pitch or the local absence of marking may potentially affect the measured phases, and thus the measured displacement and strain fields. Another point is the fact that the ideal profile of the lines should be a sine function for spectral methods. This is the optimal solution to limit noise propagation from camera sensor to final strain maps [78], and to avoid harmonics and their ensuing issues. Even though such a profile can be obtained using suitable printing devices (see [79] for instance), the line profile of fine grids obtained with usual printing techniques do not have a sine profile. In addition, the image grids are spatially sampled because of the discrete nature of complementary metal oxide semiconductor (CMOS) or charge-coupled device (CCD) sensors, and quantized by the analog-to-digital converter of the camera. This also causes an irreversible loss of information.

After marking a specimen surface with a grid and taking images while testing it, it is necessary to process the images to retrieve the displacement and strain fields, so let us now examine how to tackle this issue.

\section{Processing grid images to extract displacement and strain fields}

In this section, the link between displacement and phase change of a regular surface marking is first examined, with a special emphasis on the influence of the movement of the physical points on the final result. We also examine how to retrieve phases from a grid images. Various techniques are available but we mainly focus on one of the them: the windowed Fourier transform employed with the nominal frequency of the grid.

\subsection{Model}

In grid images, assuming that the horizontal and vertical lines of the grid are aligned with the lines and columns of pixels of the camera sensor, the light intensity $s(x, y)$ at each point $(x, y)$ is a quasi-periodic signal which can be modelled as follows [80] (in this paper, vectors are denoted by bold letters and scalars by plain letters)

$$
s(x, y)=\frac{A}{2}\left(2+\gamma \cdot \operatorname{frng}\left(2 \pi f x+\phi_{x}(x, y)\right)+\gamma \cdot \operatorname{frng}\left(2 \pi f y+\phi_{y}(x, y)\right)\right)
$$

where:

- $A$ is the average global field illumination;

- $\gamma$ is the contrast of the oscillatory pattern between 0 and 1 ;

- frng is a real $2 \pi$-periodic function with an amplitude equal to 1 and average value equal to 0 ;

- $f$ is the frequency of the carrier, defined as the inverse of the pattern pitch $p$ (that is, the inter-line distance);

- $\phi_{x}(x, y)$ and $\phi_{y}(x, y)$ are the carrier phase modulations defined modulo $2 \pi$ along the $x$ - and $y$-axes respectively. This modulation is caused by the displacement which occurs at any point. In real grids, it is also due to potential grid defects. 


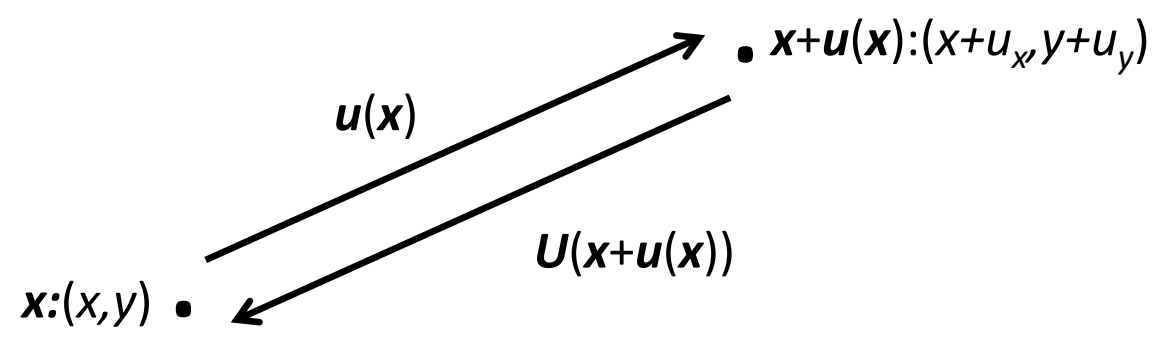

Figure 1: Displacement of a physical point (after [61])

Parameters $A$ and $\gamma$ are assumed to be constant here but they may slightly change in practice in actual images. It should also be mentioned that in real grids, the lighting intensity along dark lines is nearly constant. Pixelation causes however crossing points to be darker than points of the lines located away from the crossings. This is the reason why we adopt here a model in which two unidirectional regular line patterns are added, even though this leads this phenomenon to be more pronounced in the model than in real grid images.

A slight movement of the grid can be interpreted as a change of its phase, so displacement and phase changes are directly related. The objective of the next section is to analyse precisely this link.

\subsection{Link between displacement and phase change, strain and phase derivative change}

Sections 4.2.1 to 4.2.4 are an adaptation and an extension of [81] which discusses the restricted case of a so-called unidirectional grid (that is, a sine wave along a given direction), the phase before deformation being assumed to be null.

\subsubsection{Link between phases and displacements}

Following [81, we introduce the direct displacement field $\boldsymbol{u}=\left(u_{x}, u_{y}\right)$ and the inverse displacement field $\boldsymbol{U}=\left(U_{x}, U_{y}\right)$. We use this notation to be consistent with our preceding papers, but the reader should note that in [81, the direct displacement field is noted $U$ and the inverse displacement field is $u$. A point of coordinates $\boldsymbol{x}=(x, y)$ before deformation is mapped to the point $\boldsymbol{x}+\boldsymbol{u}(\boldsymbol{x})$ after deformation (see Figure 1), and a point of coordinates $(x, y)$ after deformation corresponds to the point of coordinates $\boldsymbol{x}+\boldsymbol{U}(\boldsymbol{x})$ before deformation. Both fields are thus linked through the following equations:

$$
\left\{\begin{array}{l}
\boldsymbol{u}(\boldsymbol{x})=-\boldsymbol{U}(\boldsymbol{x}+\boldsymbol{u}(\boldsymbol{x})) \\
\boldsymbol{U}(\boldsymbol{x})=-\boldsymbol{u}(\boldsymbol{x}+\boldsymbol{U}(\boldsymbol{x}))
\end{array}\right.
$$

or, equivalently, component-wise:

$$
\left\{\begin{array}{l}
u_{x}(x, y)=-U_{x}\left(x+u_{x}(x, y), y+u_{y}(x, y)\right) \\
u_{y}(x, y)=-U_{y}\left(x+u_{x}(x, y), y+u_{y}(x, y)\right)
\end{array}\right.
$$

and 


$$
\left\{\begin{array}{l}
U_{x}(x, y)=-u_{x}\left(x+U_{x}(x, y), y+U_{y}(x, y)\right) \\
U_{y}(x, y)=-u_{y}\left(x+U_{x}(x, y), y+U_{y}(x, y)\right)
\end{array}\right.
$$

Let $s_{1}$ and $s_{2}$ be the grid images before and after deformation, respectively, and $\phi_{x}^{1}, \phi_{y}^{1}, \phi_{x}^{2}, \phi_{y}^{2}$ the corresponding phase modulations along the $x$ - and $y$-directions.

From (4.1) and 4.2), and assuming that the illumination of the specimen does not vary during the test, we obtain:

$$
\begin{aligned}
s_{2}(\boldsymbol{x}) & =s_{1}(\boldsymbol{x}+\boldsymbol{U}(\boldsymbol{x})) \\
& =\frac{A}{2}\left(2+\gamma \cdot \operatorname{frng}\left(2 \pi f\left(x+U_{x}(x, y)\right)+\phi_{x}^{1}\left(x+U_{x}(x, y), y+U_{y}(x, y)\right)\right)\right. \\
& \left.+\gamma \cdot \operatorname{frng}\left(2 \pi f\left(y+U_{y}(x, y)\right)+\phi_{y}^{1}\left(x+U_{x}(x, y), y+U_{y}(x, y)\right)\right)\right)
\end{aligned}
$$

After identification of the phase modulations in $s_{2}$ :

$$
\left\{\begin{array}{l}
\phi_{x}^{2}(x, y)=2 \pi f U_{x}(x, y)+\phi_{x}^{1}\left(x+U_{x}(x, y), y+U_{y}(x, y)\right) \\
\phi_{y}^{2}(x, y)=2 \pi f U_{y}(x, y)+\phi_{y}^{1}\left(x+U_{x}(x, y), y+U_{y}(x, y)\right)
\end{array}\right.
$$

that is

$$
\boldsymbol{\phi}^{2}(\boldsymbol{x})=2 \pi f \boldsymbol{U}(\boldsymbol{x})+\boldsymbol{\phi}^{1}(\boldsymbol{x}+\boldsymbol{U}(\boldsymbol{x}))
$$

this equation holding modulo $2 \pi$. In [81], the grid image before deformation is supposed to be perfect, in other words, no phase modulation is needed and both $\phi_{x}^{1}$ and $\phi_{y}^{1}$ are simply disregarded. It is therefore possible to conclude in this case that the phase modulations are proportional to the components of the inverse displacement field. In the present model, which is more realistic since it accounts for the initial phase modulation caused for instance by grid defects (Section 6.6 below), this conclusion is however no longer true. Moreover, by plugging $(4.3)$ in $\phi^{2}(\boldsymbol{x}+\boldsymbol{u}(\boldsymbol{x}))$ given by (4.6) and using (4.2), we obtain the link between the phases $\phi_{x}^{2}$ and $\phi_{y}^{2}$ in the current configuration, and their counterpart $\phi_{x}^{1}$ and $\phi_{y}^{1}$ in the reference configuration. Thus:

$$
\phi^{2}(\boldsymbol{x}+\boldsymbol{u}(\boldsymbol{x}))=2 \pi f \boldsymbol{U}(\boldsymbol{x}+\boldsymbol{u}(\boldsymbol{x}))+\phi^{1}\left(\boldsymbol{x}+\boldsymbol{u}(\boldsymbol{x})+\boldsymbol{U}(\boldsymbol{x}+\boldsymbol{u}(\boldsymbol{x}))=-2 \pi f \boldsymbol{u}(\boldsymbol{x})+\phi^{1}(\boldsymbol{x})\right.
$$

Let us note $\Delta$ the operator which gives field changes, here phase changes, once mapped in the coordinate system of the grid before deformation. That is,

$$
\left\{\begin{array}{l}
\Delta \phi_{x}(x, y)=\phi_{x}^{2}\left(x+u_{x}(x, y), y+u_{y}(x, y)\right)-\phi_{x}^{1}(x, y) \\
\Delta \phi_{y}(x, y)=\phi_{y}^{2}\left(x+u_{x}(x, y), y+u_{y}(x, y)\right)-\phi_{y}^{1}(x, y)
\end{array}\right.
$$

We also note $\widetilde{\Delta}$ the operator which gives field changes once mapped in the coordinate system of the grid after deformation:

$$
\left\{\begin{array}{l}
\widetilde{\Delta} \phi_{x}(x, y)=\phi_{x}^{2}(x, y)-\phi_{x}^{1}\left(x+U_{x}(x, y), y+U_{y}(x, y)\right) \\
\widetilde{\Delta} \phi_{y}(x, y)=\phi_{y}^{2}(x, y)-\phi_{y}^{1}\left(x+U_{x}(x, y), y+U_{y}(x, y)\right)
\end{array}\right.
$$

With Equation 4.6, we have

$$
\widetilde{\Delta} \phi(\boldsymbol{x})=2 \pi f \boldsymbol{U}(\boldsymbol{x})
$$

and with Equation 4.8 ,

$$
\Delta \phi(\boldsymbol{x})=-2 \pi f \boldsymbol{u}(\boldsymbol{x})
$$




\subsubsection{Estimating the displacement field from the phase change}

Looking at Equation 4.9 , we can see that $\boldsymbol{u}$ cannot be directly estimated from $\Delta \boldsymbol{\phi}$, since this latter quantity depends itself on $\boldsymbol{u}$. This property prevents us from using out-of-theshelf techniques dedicated to profilometry, in particular those developed after the seminal work presented in [82, 83, 84. In profilometry applications, the quantity of interest is indeed the phase difference $\phi^{2}-\phi^{1}$ calculated at the same point. In this context, two approaches are possible:

- Procedure 1: the approximate, but one-step approach. The displacement $\boldsymbol{u}$ is simply estimated as

$$
\boldsymbol{u}(\boldsymbol{x})=-\frac{p}{2 \pi}\left(\phi^{2}(\boldsymbol{x})-\boldsymbol{\phi}^{1}(\boldsymbol{x})\right)
$$

instead of

$$
\boldsymbol{u}(\boldsymbol{x})=-\frac{p}{2 \pi}\left(\phi^{2}(\boldsymbol{x}+\boldsymbol{u}(\boldsymbol{x}))-\phi^{1}(\boldsymbol{x})\right)
$$

This means that the displacement of the physical points is neglected with Equation 4.13 . This rough assumption is observed to be acceptable in real cases for which the displacement remains small. Directly differentiating the displacement obtained with this approach to deduce the strain components may however become an issue when the grid features defects, as explained in the following section.

- Procedure 2: the rigorous, but iterative approach. We can see from Equation 4.14 that $\boldsymbol{u}$ is the solution of a fixed-point equation. Assuming that an initial estimation $\boldsymbol{u}^{0}$ is available (for instance with the preceding procedure), it is possible to iteratively refine $\boldsymbol{u}$ through a fixed-point algorithm. The sequence $\boldsymbol{u}^{n}(n \geq 0)$ defined by:

$$
\boldsymbol{u}^{n+1}(\boldsymbol{x})=-\frac{p}{2 \pi}\left(\phi^{2}\left(\boldsymbol{x}+\boldsymbol{u}^{n}(\boldsymbol{x})\right)-\boldsymbol{\phi}^{1}(\boldsymbol{x})\right)
$$

converges to the sought displacement $\boldsymbol{u}$. Indeed, any fixed-point algorithm $\boldsymbol{u}^{n+1}=$ $f\left(\boldsymbol{u}^{n}\right)$ is known to converge as soon as the supremum of the derivatives of $f$ (in a neighbourhood of $\boldsymbol{x}$ containing $\boldsymbol{x}_{0}$ ) is bounded by a certain constant $K<1$ (in which case Banach fixed-point theorem is satisfied since $f$ is a contraction) [85]. Moreover, the error rate of the approximation of $x$ by $x_{n}$ is bounded by $O\left(k^{n}\right)$. Here, a bound of the derivative of $f$ is given by $K=p / 2 \pi \cdot\left\|\nabla \phi_{2}\right\|_{\infty}$, where $\|\cdot\|_{\infty}$ denotes the supremum of a function. In practice, typical values for the phase derivatives are around $10^{-3}$, ensuring the very rapid convergence of the iterative algorithm, one iteration only being generally sufficient in practice in the case of small strains.

\subsubsection{Unequivocal correspondence between phases and coordinates of points}

In these procedures, phases are extracted from grid images, for instance by using the localized spectrum analysis described in Section 4.4 .2 below, but the raw phases distributions are known modulo $2 \pi$ at this stage. It means that discontinuities occur in the raw phase images as soon as the displacement in a given map has a spatial fluctuation greater than one grid pitch $p$. Such a discontinuous distribution is said to be wrapped. This phenomenon is illustrated in Figure 2. There is a broad literature describing various tools for automatically unwrapping phases, since the same problem occurs in fringe processing, [86, 87, 88, 89, 90, 91, 92, 93, 94, 95] for instance. Since some programmes are 

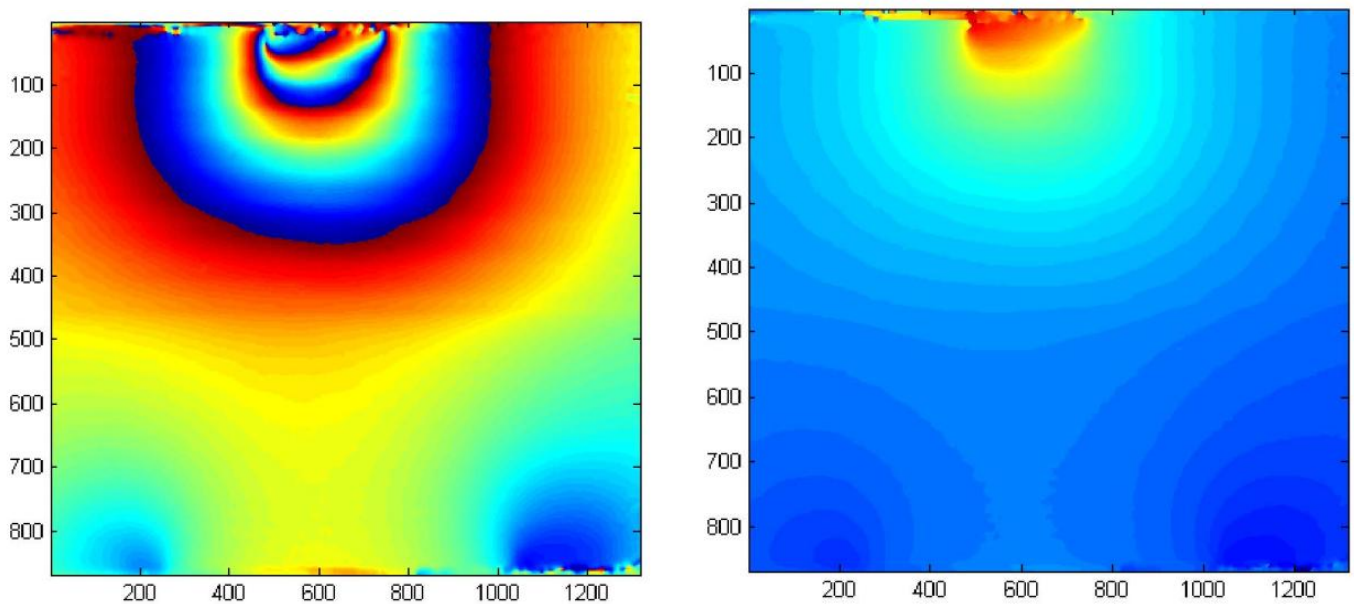

Figure 2: Illustration of wrapped and unwrapped phase distribution. Left: wrapped (revealed by several sudden color changes between red and blue); right: unwrapped.

available online on the internet, they are generally directly employed. For instance, the socalled phase-quality guided path following method and the Goldstein's branch cut method described in 90] are available in [96. The programme corresponding to the procedure described in 92 is given in 97 ] and 98 .

Phase distributions become spatially continuous after applying unwrapping (regardless to geometric discontinuities such as cracks or holes). However, they are still defined modulo $2 \pi$. Indeed, it can be a problem if the actual displacement of a given point of the specimen is greater than one grid pitch from one image to another. It means that a certain integer multiple of $2 \pi$, denoted $k$ hereafter, should be added to the phases to be sure that there is an unequivocal correspondence between the coordinates of any point of the surface under investigation after deformation, and the grid phases at that point. This can be achieved by using one of the following procedures:

- the grid is a regular marking but its aspect is slightly impaired by defects or slight heterogeneities. A procedure simply based on DIC maximisation can thus be performed between reference and current grid images to deduce $k$. Note that grid defects are useful here to ensure that minimisation is not trapped by the periodic patterns;

- the displacement of a given feature can be observed to the naked eye between reference and current phase derivative maps (continuous phase derivative maps can be obtained in any case, see [99, 80]), and $k$ can be deduced by hand. This procedure is however laborious because it is not automatic;

- a so-called temporal unwrapping can be performed if a set of images captured during a test is available. Temporal unwrapping means that a point of the phase maps is considered, and that phase jumps detected between two consecutive images considered at this point are then used to correct the whole phase map. 


\subsubsection{Estimating the strain components}

The quantity of interest is often the linear strain tensor, whose components write:

$$
\epsilon_{i j}=\frac{1}{2}\left(\frac{\partial u_{i}}{\partial j}+\frac{\partial u_{j}}{\partial i}\right) \quad i, j=x, y
$$

This quantity can be merely directly estimated by numerically differentiating the displacement field obtained with Procedure 1 (Equation 4.13) or Procedure 2 (Equation 4.14).

If we consider now the closed-form expression of these quantities to see the errors made by using Procedure 1 instead of Procedure 2 to calculate first the displacement, and then deduce the strain, we have the following results:

- With displacements calculated with procedure 1:

From Equation 4.13 , we obtain

$$
2 \epsilon_{i j}(x, y)=-\frac{p}{2 \pi} \times\left(\frac{\partial \phi_{i}^{2}}{\partial j}(x, y)-\frac{\partial \phi_{i}^{1}}{\partial j}(x, y)+\frac{\partial \phi_{j}^{2}}{\partial i}(x, y)-\frac{\partial \phi_{j}^{1}}{\partial i}(x, y)\right) \quad i, j=x, y
$$

- With displacements calculated with procedure 2:

From Equation 4.14, in which $\boldsymbol{u}$ is obtained at convergence of Procedure 2, we obtain

$$
2 \epsilon_{i j}=-\frac{p}{2 \pi}\left(\frac{\partial \Delta \phi_{i}}{\partial j}+\frac{\partial \Delta \phi_{j}}{\partial i}\right) \quad i, j=x, y
$$

Let us compare these two estimations. The phase derivatives involved in Equations 4.18 (and thus in Equation 4.9) can be expressed by applying the chain rule:

$$
\begin{aligned}
\frac{\partial \Delta \phi_{i}}{\partial j}(x, y) & =\left(\frac{\partial x}{\partial j}+\frac{\partial u_{x}}{\partial j}(x, y)\right) \frac{\partial \phi_{i}^{2}}{\partial x}\left(x+u_{x}(x, y), y+u_{y}(x, y)\right) \\
& +\left(\frac{\partial y}{\partial j}+\frac{\partial u_{y}}{\partial j}(x, y)\right) \frac{\partial \phi_{i}^{2}}{\partial y}\left(x+u_{x}(x, y), y+u_{y}(x, y)\right) \\
& -\frac{\partial \phi_{i}^{1}}{\partial j}(x, y) \quad i, j=x, y
\end{aligned}
$$

To ease the comparison with the results obtained with Procedure 1, we consider the particular case $i=j=x$. With Procedure 1, we have

and with Procedure 2

$$
\epsilon_{x x}=-\frac{p}{2 \pi} \times \frac{\partial \Delta \phi_{x}}{\partial x}
$$

$$
\begin{aligned}
\epsilon_{x x}(x, y) & =-\frac{p}{2 \pi} \times\left(\left(1+\epsilon_{x x}(x, y)\right) \frac{\partial \phi_{x}^{2}}{\partial x}\left(x+u_{x}(x, y), y+u_{y}(x, y)\right)\right. \\
& \left.+\frac{\partial u_{y}}{\partial x}(x, y) \frac{\partial \phi_{x}^{2}}{\partial y}\left(x+u_{x}(x, y), y+u_{y}(x, y)\right)-\frac{\partial \phi_{x}^{1}}{\partial x}(x, y)\right)
\end{aligned}
$$

By comparing the expressions obtained with Equations 4.20 and 4.21 , we can see that two types of approximations are induced in strain calculation with Procedure 1: 
- the derivatives of $u_{x}$ and $u_{y}$ are not taken into account;

- the subtraction between the phases derivatives is not performed at the same physical point.

The first approximation does not really induce a significant error as long as the normal strain remains much lower than one, and the local in-plane rotation is small, which is generally the case. It is observed in practice that the second approximation is much more critical in the presence of grid defects such as grid pitch fluctuations or even slight local lack of ink. Indeed, even with small displacements (lower than the grid pitch), the disturbance induced in the phase derivative maps is not eliminated by subtracting current and initial phase derivative distributions. This potentially induces fictitious strain to appear in final strain maps while the impact on displacement maps is generally negligible. Depending on the amplitude of the grid defects and the amplitude of the actual and sought strain components, this can be really a problem, as illustrated in Section 6.6 below.

Note finally that the two preceding assumptions amount to identify $\boldsymbol{u}(x, y)$ and $-\boldsymbol{U}(x, y)$ in Equation 4.2 , yielding:

$$
\epsilon_{i j}=-\frac{1}{2}\left(\frac{\partial U_{i}}{\partial j}+\frac{\partial U_{j}}{\partial i}\right) \quad i, j=x, y
$$

Consequently, with Equation 4.11

$$
2 \epsilon_{i j}=-\frac{p}{2 \pi}\left(\frac{\partial \widetilde{\Delta} \phi_{i}}{\partial j}+\frac{\partial \widetilde{\Delta} \phi_{j}}{\partial i}\right) \quad i, j=x, y
$$

This means that the strain components can also be computed with $\widetilde{\Delta}$ in this case.

\subsubsection{Other techniques used in the literature to calculate strain components}

As explained in the preceding section, some assumptions can be reasonably made to simplify the practical calculation of the strain components from Equations 4.18 and 4.19 . In particular, we can simplify Equation 4.19 into

$$
\frac{\partial \Delta \phi_{i}}{\partial j}=\Delta \frac{\partial \phi_{i}}{\partial j} \quad i, j=x, y
$$

which means that the strain components can be inferred from the phase derivative changes with the following equation

$$
2 \epsilon_{i j}=-\frac{p}{2 \pi} \times\left(\Delta \frac{\partial \phi_{i}}{\partial j}+\Delta \frac{\partial \phi_{j}}{\partial i}\right) \quad i, j=x, y
$$

This is the procedure employed in [99, 80, in which the $\Delta$ operator (mapping the phase derivative distributions of the current grid image in the coordinate system of the grid before deformation) is performed by using the displacement field obtained with Procedure 1 as described in Equation 4.13, regardless of the procedure employed to retrieve the phase distributions (several strategies are potentially available) the main merit of this approach is to perform the subtraction of the phase derivative maps at the same physical points, the movement between current and reference grid images being compensated. This enables us to get rid of the consequence of the grid defects in the strain maps, and thus to obtain a good compromise between strain resolution and spatial resolution (these quantities are thoroughly defined in Section 5), as illustrated in several examples shown in Section 7. 
Beside this approach for strain calculation and as a general remark, it is worth mentioning that approaches based on local displacement smoothing (with polynomials for instance) are widely employed in photomechanics: displacements are estimated at some points, which are then considered as the nodes of a mesh built up by the user. The displacement is then locally smoothed or interpolated by functions which are derived to provide the strain components. This is a classic approach in subset-based DIC because of the iterative nature of this technique. This is not a drawback in cases for which the strain gradients remain "low" in the actual distribution. The same remarks holds when constitutive parameters are identified from full-field measurements: these parameters are in general global and represent mean values over wide regions, often the whole specimen itself. Consequently, relying on a huge amount of data is not really relevant in this case: this would only reduce noise issues in the identified parameters. In addition to large computation times, this also potentially leads to huge data files to be stored, handled and processed, which should obviously be avoided when it is not necessary. When considering functionally graded materials (thus spatially changing mechanical properties), or cases for which high strain gradients occur in the tested specimen (e.g. near a crack tip), the conclusion is obviously different and one should avoid any loss of information. In addition, assessing the metrological performance of the grid method is much easier if no interpolation is performed, as illustrated in Section 5. No result on the metrological performance of the grid method with interpolation seems to be available in the literature, even though recent results obtained in DIC could certainly be adapted [100, 101]. The reader interested in approaches based on local smoothing to obtain strain fields from full-field measurement is referred to the broad literature on DIC [10], or to [102, 103, 104] for instance, where the mesh is adapted to account for strain gradient intensity. Indeed, the displacements can potentially be obtained with any full-field technique with such procedures. Consequently, techniques initially developed for DIC for instance are also applicable to displacements fields obtained with the grid method.

\subsection{Retrieving phase and phase derivatives from grid images}

The change in phase between reference and current configurations being linked to the displacement components according to Equation 4.14, it is necessary to retrieve first the phases from grid images taken in both configurations. This is a problem similar to that of phase retrieval from fringes obtained in optical metrology using for instance interferometric setups or fringe projection profilometry. The basic idea is that phase information is concentrated in (usually spread) spikes in the spectrum of a fringe image. When the spikes are well separated, the phase can be retrieved with a pass-band filter. Designing this filter is however not an easy task. There is a broad literature available on this topic and various tools have been proposed. The main ones are the Fourier Transform (FT) [105, 106], the WFT [107, 108, 109] and wavelet transform [110, 111, 112]. Local techniques such as the WFT or wavelet transform permit the user to overcome limitations caused by, for instance, an uneven illumination of the specimen.

It seems that only the FT and the WFT were employed and adapted to process grid images. The reason is that wavelet tranform is not adapted here because the frequency of the carrier is known a priori and is only slightly modulated by straining. The use of the FT in the particular case of grids is mentioned in [113, 114, 72, 115, 116, 117, 118, 64, 119] or in [120, 32, 33] for instance. In these last references, this gave birth to the so-called Geometric Phase Analaysis (GPA) briefly described in Section 4.3. The use of the WFT in the case of grids in mentioned in [121, 122].

Alternative methods or techniques derived from the above ones also exist. In [123] for 
instance, a correlation function is minimized to estimate the degree of similarity between real fringes and a suitable mathematical model. More recently, the so-called sampling moiré was proposed in Ref. [74]. It consists in introducing a fictitious phase shifting by sub-sampling grid images. So-called "thinned-out" grid images are obtained after this procedure, each of them being shifted from the others. The authors propose then to interpolate these thinned-out grid images to recover the initial pixel density of the grid images, which induces an approximation. Classic phase extraction from several images of phase-shifted fringes is then applied. The errors made when using this technique are discussed in [124].

A comparison of some metrological characteristics of various techniques applied to grid images processing is given in [125], especially in terms of bias and spatial resolution. A comparison between FT, WFT and wavelet transform is also proposed in [126] in the case of profilometry. It is concluded that both the FT and the WFT lead to more robust algorithms than wavelet transform. For grid image processing, we are in a particular case for which the frequency carrier is known a priori, so we are only interested in retrieving the slight modulation of this carrier. Since the grid method is generally used for measuring "small" strain components, the modulation of the regular grid pattern remains low. This leads the changes in the regular line network due to straining to be generally much smaller than those observed in the case of profilometry or interferometry for instance. Compared to these two techniques, another feature of the grid method is the fact that in practice, the number of "fringes" to be processed with grids is much higher since this is directly the number of the grid lines. For instance, there are often so many lines in a picture that they are not distinguishable to the naked eye. This is generally not the case with fringe patterns obtained with the two aforementioned techniques. Finally, it is worth mentioning that two crossed line patterns must be processed simultaneously in the case of bidirectional grids.

\subsection{Fourier and windowed Fourier transforms}

\subsubsection{General case}

Both the FT and the WFT provide a complex number map denoted $\widehat{s}(\xi, \eta)$ and $\widehat{s_{W}}(u, v, \xi, \eta)$. Both are defined for a pair of frequencies $\xi, \eta$ along the $x$ - and $y$-directions, respectively, the WFT being defined in addition at a given point of coordinates $(u, v)$. The FT is defined as follows

$$
\widehat{s}(\xi, \eta)=\int_{-\infty}^{+\infty} \int_{-\infty}^{+\infty} s(x, y) e^{-2 i \pi(\xi x+\eta y)} \mathrm{d} x \mathrm{~d} y
$$

The WFT is the local version of the FT, which means that the FT is calculated for a zone surrounding any point $M(u, v)$ of the image. This zone is defined by the analysis window $g$. The WFT is defined as follows

$$
\widehat{s_{W}}(u, v, \xi, \eta)=\int_{-\infty}^{+\infty} \int_{-\infty}^{+\infty} s(x, y) g(u-x, v-y) e^{-2 i \pi(\xi u+\eta v)} \mathrm{d} x \mathrm{~d} y
$$

The integrands above involve several functions:

1. the signal $s$ to be processed

2. a modulation of frequencies $\xi, \eta$ along the $x$ - and $y$ - directions, respectively

3. and for the WFT only, a 2D window function $g$ whose width is driven by a parameter chosen by the user. This function is symmetric, positive, and integrates to 1 
The inverse of each of these transforms is defined as follows:

- for the inverse of the FT (denoted IFT hereafter):

$$
s(x, y)=\int_{-\infty}^{+\infty} \int_{-\infty}^{+\infty} \widehat{s}(\xi, \eta) e^{2 i \pi(\xi x+\eta y)} \mathrm{d} \xi \mathrm{d} \eta
$$

- and for the inverse of the WFT (denoted IWFT hereafter):

$$
s(x, y)=\int_{-\infty}^{+\infty} \int_{-\infty}^{+\infty} \int_{-\infty}^{+\infty} \int_{-\infty}^{+\infty} \widehat{s_{W}}(u, v, \xi, \eta) g(x-u, y-v) e^{2 i \pi(\xi u+\eta v)} \mathrm{d} u \mathrm{~d} v \mathrm{~d} \xi \mathrm{d} \eta
$$

Retrieving the phase distributions (one along the $x$-direction, one along the $y$-direction) of a grid image using the FT consists in calculating the FT of this image, filtering the corresponding spectrum by considering in turn a zone surrounding the peaks of each of the first harmonics $(f, 0)$ and $(0, f)$, performing the IFT of each of these zones, and finally extracting the phase distribution of each result [64, 120, 32, 33, 119]. This procedure should be applied to both the reference and the current grid images to deduce the phase change between these two configurations. This is the spirit of the GPA.

Similarly, retrieving the phase of a regular pattern such as fringes [107, 108, 109] or grids [122] by using the WFT consists first in selecting a zone in the Fourier domain over which integration is performed. As in the preceding case, this can be done by considering in turn a zone surrounding the peaks of each of the first harmonics $(f, 0)$ and $(0, f)$. The preceding procedure is then applied up to phase determination. Compared to the preceding case, it is applied in turn at any point/pixel of the image, the window $g$ sliding over the whole image. The integration domain is here much smaller than in the preceding case since it is bounded by the window. Compared to FT, this WFT-based procedure takes however much more time, as reported in [122].

Much earlier on (but not cited in [122]), Surrel proposed to apply the WFT with two sole points in the frequency domain: $(f, 0)$ and $(0, f)[121$. This can be considered as a particular and ultimate case of the more general WFT described in [122. Even though other frequencies than the nominal frequency of the grid are not taken into account for this particular case, this option leads to very good results, as illustrated in the examples discussed in Section 7. The benefit is also to have a much shorter calculation time since only one frequency is considered for each of the two directions and, last but not least, the fact that calculations are tractable to get a priori estimate for the metrological performance in terms of spatial resolution, measurement resolution and bias (these quantities are thoroughly defined below). This point is crucial for future standardisation of full-field measurement techniques. For all these reasons, we focus now on the WFT in the particular case $(f, 0)$ and $(0, f)$. This procedure is named the Local Spectrum Analysis (LSA) in the following to clearly distinguish it from the GPA.

\subsubsection{The Local Spectrum Analysis}

For $(f, 0)$ and $(0, f)$, the WFT writes as follows 


$$
\begin{cases}\text { along direction } x: & \widehat{s_{W}}(u, v, f, 0)=\int_{-\infty}^{+\infty} \int_{-\infty}^{+\infty} s(x, y) g(u-x, v-y) e^{-2 i \pi f x} \mathrm{~d} x \mathrm{~d} y \\ \text { along direction } y: & \widehat{s_{W}}(u, v, 0, f)=\int_{-\infty}^{+\infty} \int_{-\infty}^{+\infty} s(x, y) g(u-x, v-y) e^{-2 i \pi f y} \mathrm{~d} x \mathrm{~d} y\end{cases}
$$

We can see that, $g$ being symmetric, $\left.\widehat{s_{W}}(u, v, f, 0)\right)$ and $\left.\widehat{s_{W}}(u, v, 0, f)\right)$ are the convolution of $g$ with $s(x, y) e^{-2 i \pi f x}$ and $s(x, y) e^{-2 i \pi f y}$ respectively, which is a fast calculation with the Fast Fourier Transform (FFT) algorithm. The phase distributions $\phi_{x}(x, y)$ and $\phi_{y}(x, y)$ for a grid image are estimated modulo $2 \pi$ by calculating the arguments of the complex numbers defined Equation 4.30, which gives [81]

$$
\left\{\begin{array}{l}
\phi_{x}(x, y)=\arctan \left(\frac{\operatorname{Im}\left(\widehat{s_{W}}(u, v, f, 0)\right)}{\operatorname{Re} \widehat{s_{W}}(u, v, f, 0)}\right) \\
\phi_{y}(x, y)=\arctan \left(\frac{\operatorname{Im}\left(\widehat{s_{W}}(u, v, 0, f)\right)}{\operatorname{Re}\left(\widehat{s_{W}}(u, v, 0, f)\right.}\right)
\end{array}\right.
$$

Concerning the shape of the analysis window $g$, it is proposed in [121] to employ a triangle spanning two grid periods. Phase extraction with the discrete version of the WFT is indeed regarded as a spatial phase shifting in [121. This procedure is an extension of the classic phase shifting procedure employed to extract phases from fringes provided for instance by interferometric setups [127]. In the latter case, a mirror is slightly translated with a piezoelectric stage. This induces a change in the optical path, thus a delay and eventually a phase shift. With the spatial version of phase shifting, the pixels surrounding the pixel at which the phase is calculated are considered as containing all the same phase, plus a multiple of a constant phase shift equal to $\frac{2 \pi}{N}$, where $N$ is the number of pixels used to encode one grid period. Considering spatial phase shifting enables one to use or adapt tools and results obtained with the temporal phase shifting procedure employed to process fringes, see [127, 128, 81, 129, 121, 130, 131, 132, 133, 134, 135, 136] for instance. Concerning the phase shifting problem, Ref. [121] mentions that a triangular window provides an information insensitive to harmonics up to the order $N-2$. It also minimizes the effect of miscalibration, which occurs when $N$ is not exactly an integer value. This is generally the case in practice because it is not possible to perfectly adjust the number of pixels per grid period in grid images. In addition, this value slightly changes during deformation of the grid during a test. For bidirectional grids, it is proposed in [121] to separate the information between the $x$ - and $y$-directions by spatially averaging over a length equal to the pitch of the grid to be eliminated, and then to apply in turn a unidirectional phase extraction along each direction. For instance, to extract the phase $\phi_{x}$ at a given pixel, $N$ consecutive portions of lines of pixels surrounding this pixel are averaged along direction $y$. This procedure eliminates the periodicity along direction $y$ while that along direction $x$ is preserved, which enables phase extraction along $x$.

In many applications, strain values are needed and they are obtained by spatially differentiating the phase change measured between reference and current configurations of the specimen. Phases obtained with the procedure described above being noisy, it is generally necessary to filter them prior to differentiation. In [99, 80], it is thus proposed to employ a Gaussian analysis window wider than the triangular one to filter noise more efficiently while extracting the phase. In addition, a triangle may lead to some issues if the width of the analysis window is not an integer value [137. Some authors employ a Gaussian analysis window on the ground that it minimizes the Heisenberg box [108, 138. Finally, 
the Gaussian window is derivable, which permits direct extraction of phase derivatives without calculating the phases if necessary, merely by commuting the derivation from the signal to the window when calculating the derivatives from Equation 4.30 [80. This can be useful in case of phase jumps in the phase distribution but increases the calculation time.

We propose now to address the metrological performance of the grid method by considering the WFT localized at $(f, 0)$ and $(0, f)$, and assuming the a Gaussian analysis window is employed in the WFT, results obtained with other types of windows being discussed in [137].

\section{Metrological performance}

\subsection{Introduction}

An important remark is the fact that we deal here with a measurement issue. However, despite many efforts on this subject, see [139, 140, 141, 142, 143, 144, 145, 146] for instance, one standard only: ASTM-E2208 [147] seems to be available to help users estimate the metrological performance of full-field measurement techniques, as well as a draft standard for dynamic measurements [148. Ref. [147] only gives general ideas on this subject, which illustrates the trickiness of this problem. Assessing the errors made while measuring displacement or strain components is addressed in several papers on DIC, see [149, 150, 151, 152, 153, 154, 155, 156, 157, 158, 100, 101] for instance. This issue is discussed also in Refs. [124, 75] concerning the sampling moiré. We propose here an in-depth discussion on this point. According to [147], sources of errors can be divided into two categories: those related to the experimental setup and imaging, and those due to the calculation of the desired quantities from the images. We mainly focus here on the latter, in the case of phase extraction from grid images by WFT, and in the particular case for which only the nominal frequency of the grid is considered, calculations being tractable in this case. A normalised isotropic Gaussian analysis window is used here, as justified above, but similar expressions could be obtained with other classic windows used in the WFT [137]. This Gaussian window is defined by

$$
g(x, y)=\frac{1}{2 \pi \sigma^{2}} e^{\left(-\frac{x^{2}+y^{2}}{2 \sigma^{2}}\right)}
$$

where $\sigma$ is the standard deviation of the Gaussian function. The following results are obtained by extending some metrological properties recently presented in Ref. [78].

\subsection{Systematic and random errors}

The measurement of displacement and strain components at a given point, denoted here respectively $u_{i}, \varepsilon_{i j}, i, j \in(x, y)$, can be split into three different parts:

$$
\left\{\begin{array}{l}
u_{i}=\widetilde{u}_{i}+b_{i}^{u}+e_{i}^{u} \\
\varepsilon_{i j}=\widetilde{\varepsilon}_{i j}+b_{i j}^{\epsilon}+e_{i j}^{\epsilon}, \quad \forall i, j \in\{x, y\}
\end{array}\right.
$$

where

- $\widetilde{u_{i}}, \widetilde{\varepsilon}_{i j}$ are the reference (and sought) values 
- $b_{i}^{u}$ and $b_{i j}^{\epsilon}$ represent the bias as defined in [159, 160, i.e. the estimate of the systematic measurement error

- $e_{i}^{u}, e_{i j}^{\epsilon}$ represent the zero-mean random part of the measurements.

$b_{i}^{u}+e_{i}^{u}$ and $b_{i j}^{\epsilon}+e_{i j}^{\epsilon}$ represent the measurement errors for the displacement and the strain, respectively. These quantities may change from one component to another. This is the reason why we employ indices in the notations.

As far as quantitative strain measurements are concerned, the user is seeking $\widetilde{\varepsilon}_{i j}$, which means that $b_{i j}$ and $e_{i j}$ must be correctly estimated to determine an uncertainty range, if not reducing them by smoothing the data for instance. It is worth mentioning that $b_{i j}$ and $e_{i j}$ are not really intrinsic to the measuring method discussed here:

- $b_{i j}$ may be due to various causes. In practice a miscalibration of the parameters driving grid image processing (for instance the value of the pitch of the grid), image distortions caused by the lens or out-of-plane movements may induce a systematic error in the maps, which is the case for any in-plane measuring tool [161, 162, 163]. Image distortion is discussed in Section 6.4 below. Concerning the influence of outof-plane movement on strain maps, it is shown in [61] that a change $\delta d$ in the distance $d$ between specimen and camera induces a parasitic strain equal to $\varepsilon=-\frac{\delta d}{d}$. We do not consider the miscalibration of the parameters, but the bias due to image processing itself. Thus we will focus only on this last issue in this section. The most striking point is that this bias depends on the local distribution of the displacement or the strain itself, more precisely the "sharpness" of the details in the distribution to be revealed at a given point. Hence $b_{i j}$ depends on the local nature of the measurand: the bias is signal-dependent, which makes it difficult to assess in practice. In the general case, this is a spatially changing quantity, and thus not a unique scalar which would ideally model this property. It is however possible to assess $b_{i j}$ in synthetic cases for which a reference strain field is known a priori, as discussed in Section 5.4.2.

- $e_{i j}$ is a random quantity, so statistics must be employed to model and describe it correctly. It depends on extrinsic parameters such as grid contrast or lighting intensity. Since lighting is often not constant throughout grid images, the characteristics of this noise, especially its standard deviation, generally evolves throughout strain maps. Other phenomena induce errors such as thermal effects, convection with ambiant air, vibrations, lighting flickering or calculating/truncation errors for instance. They are however difficult to quantify and often neglected or incorporated in the random part of the error, so they are not considered in the present study.

\subsection{Definition of the metrological performance parameters}

Following the preceding comments, we will mainly focus here on three important parameters describing the metrological performances of the grid method, namely the measurement resolution, the bias and the spatial resolution. They are briefly defined or recalled hereafter.

\subsubsection{Measurement resolution}

In Ref. [159], the measurement resolution is defined by the smallest change in a quantity being measured that causes a perceptible change in the corresponding indication. More precisely, it is proposed in [164] to define it as the change in quantity being measured that 
causes a change in the corresponding indication greater than one standard deviation of the measurement noise, which enables us to quantify it. This definition is quite arbitrary, any other (reasonable) multiple of the standard deviation being also potentially acceptable, but the idea is that the resolution quantifies the smallest change not likely to be caused by measurement noise 164. We focus here on camera sensor noise which propagates to the final displacement or strain maps through the image processing procedure which is employed. This is often the main cause of noise in final displacement and strain maps, and it is also possible to analyze this noise from a theoretical point of view. Some other causes of disturbance may occur in practice:

- Quantization also induces a noise, but it is generally lower than the preceding one, and thus not considered here. The interested reader is referred to [135] for additional information on this problem.

- Another cause of disturbance is the presence of grid defects. These defects are pitch fluctuation or local lack of marking. These defects are discussed in Section 6.6 and it is shown that there impact on the displacement and strain fields can be eliminated to a large extent, but it is difficult to model the residual which remains after this correction, and thus to predict their impact on measurements: they can only be assessed through real experiments.

- Aliasing or a slight moiré phenomenon between grid and camera sensor may also occur. They cause parasitic fringes to appear, but this phenomenon is difficult to master and thus even more to model because it depends on various settings and camera features. This point is discussed in Sections 6.1 and 6.7.

- Finally, the quality of the displacement and strain maps also depends on the pixel density chosen to encode one grid pitch but again, this point is difficult to model.

In conclusion, we study here the measurement resolution from a theoretical point of view, so we consider that the camera sensor is the sole cause of random noise in displacement and strain maps.

\subsubsection{Measurement bias}

As explained above, the bias due to grid image processing depends on the actual distribution of the quantity to be determined (displacement or strain). A classic way to assess the bias is to employ a mere synthetic sine function. This bias is then estimated as a function of the frequency of this sine, as in Ref. [165, 166, 167, 168] for DIC and in [125, 169] for methods based on phase extraction. The bias is here equal to the loss of amplitude of this sine function which models the reference distribution. We consider here the quantity denoted $\lambda$ defined as the ratio between this loss of amplitude and the amplitude of the reference sine function itself. This normalised bias, called hereafter bias for the sake of convenience, is thus a positive number systematically lower than one.

\subsubsection{Spatial resolution}

We propose here two definitions of the spatial resolution. With the first definition, the spatial resolution denoted $d_{1 j}, j \in\{u, \epsilon\}$ is defined as the shortest distance between two spatially independent measurements. This definition has been adopted for instance in [78]. Since a window is used in the WFT, the spatial resolution can be defined by the width of this window. For a triangle, this is the base of the triangle. For a Gaussian window, this 
is a multiple of the standard deviation of the Gaussian window. According to the classic $3-\sigma$ rule [170, this width is considered to be equal to $6 \times \sigma$. This choice is quite arbitrary and other multiples of the standard deviation could be considered. This definition of the spatial resolution holds here for the phase, and therefore for the displacement. It also holds for the phase derivatives and the strain components if no smoothing is performed before deriving the phases and the displacements, respectively. Otherwise spatial resolution is all the more impaired as the width of the filter is significant.

We can see that this definition is quite arbitrary in the case of the Gaussian analysis window. Hence we propose a second definition suggested in [167], after similar defintion proposed in [165]. The spatial resolution denoted $d_{2 j}, j \in\{u, \epsilon\}$ is defined here as the lowest period of a sinusoidal deformation that the technique is able to reproduce before losing a certain percentage of amplitude, in other words before the bias reaches a certain value, which must be chosen a priori. The advantage of the second definition is to not directly depend on the size of the region surrounding the pixel at which each measurement is obtained, this size being not always clearly defined. A limitation is the fact that in some cases, the loss of amplitude is not a monotonic function of the period of the aforementioned signal, which can potentially lead to ambiguous values for the spatial resolution with this definition. This is for instance the case of some types of windows different from the Gaussian one. This point is discussed further in [137].

\subsection{Relationship between the parameters}

\subsubsection{Introduction}

It is worth emphasizing that the aforementioned quantities are linked. In addition, since the bias is linked to the actual value for the strain itself, it is not intrinsic to the measurement technique. Finally, we deal here with a full-field measurement technique. This causes the spatial resolution to be introduced, which is unusual with classic measuring tools providing scalar quantities. This is one of the reasons why it is difficult to have a clear view on the metrological performance of full-field measurement techniques.

The link between these parameters can be illustrated as follows:

- improving the spatial resolution (i.e. reducing the distance between independent measuring points) leads to impair the measurement resolution (i.e. to increase the noise level in the measurements). This corresponds to an intuitive result: when decreasing the size of the window employed in the WFT, the number of pixels employed to retrieve the phases and their derivatives decreases. Hence we focus on a zone which decreases in size, which leads theoretically to reveal smaller actual events that occur in the displacement or strain distribution over the specimen under consideration. It means that the spatial resolution is improved. At the same time however, the integrals calculated in the WFT rely on a decreasing number of pixels. Since the grey level at each pixel is impacted by sensor noise, the integrals are all the more affected by noise as the number of pixels involved in their calculation decreases, impairing the measurement resolution in proportion. It means that the standard deviation of the $e_{i j}$ distribution in Equation 5.2 increases.

- in the same way, improving the spatial resolution (thus reducing the distance between independent measuring points) leads to decrease the bias: the value given by the WFT is less suffering from a local "averaging effect" since the integrals of the WFT are calculated over smaller zones. Consequently and regardless of the noise, the 
measured value becomes closer to the actual and sought value, and consequently the bias diminishes and the trueness increases.

A positive point is that for the grid method, these subtle, interconnected but intuitive phenomena can be quantified by closed-form equations which are summarized in the following section.

\subsubsection{Link between relative bias and spatial resolution in case of synthetic sinusoidal distributions}

As proposed in other papers dealing with the metrological performance of full-field measuring techniques [165, 167, 169], we propose here to consider independent reference displacement or strain distributions modeled each by a sine function

$$
\left\{\begin{array}{l}
\widetilde{u}_{y}(x, y)=A \sin \left(2 \pi f^{\prime} x\right) \\
\widetilde{\epsilon}_{y y}(x, y)=B \sin \left(2 \pi f^{\prime} x\right)
\end{array}\right.
$$

where $A, B$ are any real constants. $f^{\prime}$ is the frequency of the sine functions ( $f$ is the grid frequency). We deal here with a simulation, so no defect impacts the initial phase distribution, which can thus be considered as null. Consequently, displacement $u_{y}$ linearly depends on the phase $\Phi_{y}$ (instead of being proportional to the difference between current and reference phases), and strain $\epsilon_{y y}$ is directly proportional to the phase derivative $\frac{d \Phi_{y}}{d y}$. $\widetilde{u}_{y}$ and $\widetilde{\epsilon}_{y}$ are here completely independent and disconnected, describing each a synthetic state of displacement/strain: the displacement ensuring a constant strain amplitude in the second case is $\widetilde{u}_{y}=B /(2 \pi) f^{\prime} \sin \left(2 \pi f^{\prime} x\right)$, but it does not exhibit a constant amplitude. Such reference distributions modulating each a grid, the question is to know how these signals are returned by the WFT for these two grid images. It has been shown in [171] that at first approximation and regardless of the pixellation of the actual signal, the phases extracted with the WFT are equal to their actual counterparts convolved by the window of the WFT. The same remark holds for the displacement, the phase derivatives and the strain components. If the image is noisy, a spatially correlated Gaussian noise, whose covariance can be characterized, must be added to the phase or phase derivative maps. The variance of this noise is discussed in the following section. In the current case, since we consider a noiseless reference distribution, we have

$$
\left\{\begin{array}{l}
u_{y}=\widetilde{u}_{y} * g \\
\epsilon_{y y}=\widetilde{\epsilon}_{y y} * g
\end{array}\right.
$$

It means that in the case of an actual displacement or strain distribution modelled by a sine function, we just have to calculate the convolution of this sine by the analysis window of the WFT to get the result. This simple calculation, carried out in Ref. [137] for various windows used in the WFT, shows that the WFT returns a sine distribution whose amplitude $A^{\prime}$ is lower than $A$ since $0<\lambda<1$. For instance, for a Gaussian window of standard deviation $\sigma$, we have for the two aforementioned displacement and strain distributions

$$
\left\{\begin{array}{l}
A^{\prime}=A \times e^{-2 \pi^{2} \sigma^{2} f^{\prime 2}} \\
B^{\prime}=B \times e^{-2 \pi^{2} \sigma^{2} f^{\prime 2}}
\end{array}\right.
$$

The relative bias denoted $\lambda$ as defined in Section 5.3.1 is therefore equal to:

$$
\lambda=1-e^{-2 \pi^{2} \sigma^{2} f^{\prime 2}}
$$


Results obtained with other analysis windows are discussed in [137.

Note that in real configurations, the displacement gradients are in general much less marked than the strain gradients. It means that for a given size of the analysis window, the convolution by the analysis window mainly affects in practice strain maps, not really displacement maps. This is visible to the naked eye by a blur which is much more marked in actual measured strain maps than in the corresponding measured displacement maps. Consequently and for the sake of brevity, we mainly focus in the following on strain maps only, but similar results are obtained with displacement maps.

Equation 5.6 provides the link between the bias $\lambda$ and the spatial resolution. With the first definition given in Section 5.3. we just have to substitute $\sigma$ by $\sigma=\frac{d_{1 \epsilon}}{6}$ in Equation 5.6 . and with the second definition, $f^{\prime}$ by $\frac{1}{d_{2 \epsilon}}$, where $f^{\prime}$ is the highest frequency of the sine distribution the method is able to reproduce with a given bias equal to $\lambda$. For the first definition, this leads to

$$
\left\{\begin{aligned}
d_{1 \epsilon} & =\frac{3 \sqrt{2}}{\pi f^{\prime}} \sqrt{-\log (1-\lambda)}, \text { with } 0<\lambda<1 \\
& \text { or, conversely } \\
\lambda \quad & =1-e^{-\frac{\pi^{2} f^{\prime 2} d_{1 \epsilon}^{2}}{18}}
\end{aligned}\right.
$$

With the second definition, we deduce from Equation 5.6

$$
\left\{\begin{aligned}
d_{2 \epsilon} & =\frac{\sqrt{2} \pi \sigma}{\sqrt{-\log (1-\lambda)}}, \text { with } 0<\lambda<1 \\
& \text { or, conversely } \\
\lambda \quad & =1-e^{-2 \pi^{2} \frac{\sigma^{2}}{d_{2 \epsilon}^{2}}}
\end{aligned}\right.
$$

$f^{\prime}$ shall be regarded as a parameter in Equations 5.7, and $\lambda, \sigma$ as parameters in Equations 5.8.

We can easily deduce from Equation 5.6 the following link between $d_{1 \epsilon}, d_{2 \epsilon}$ and $\lambda$ :

$$
\frac{d_{1 \epsilon}}{d_{2 \epsilon}}=\frac{3 \sqrt{2}}{\pi} \sqrt{-\log (1-\lambda)}, \text { with } 0<\lambda<1
$$

The same equation can be written for the link between $d_{1 u}$ and $d_{2 u}$. From Equations 5.7 and 5.8, it is worth mentioning that $d_{1 \epsilon}$ and $d_{2 \epsilon}$ evolve in an opposite way as a function of $\lambda$. This is logical: increasing the bias (thus $\lambda \rightarrow 1$ ) for a given frequency $f^{\prime}$ means that the size of the analysis window used in the WFT increases, thus that the distance between two independent measurements increases too, so $d_{1 \epsilon}$ increases with $\lambda$. On the contrary, increasing the bias for a given width of the window means that the frequency of the signal increases, so that its inverse (which is $d_{2 \epsilon}$ according to its definition) decreases. As a result, the ratio between $d_{1 \epsilon}$ and $d_{2 \epsilon}$ increases as $\lambda$ increases, as shown in Figure 3 .

The effect of the bias can be visually estimated on the simulation shown in Figure 4. Figure 4 a shows a simulated phase evolution $\Phi_{y}$. It is such that $\varepsilon_{y y}$ keeps the same amplitude (here $10^{-3}$ ) over the whole map. For each column, the period of the sine component along the $y$-direction is constant while this period gently and linearly increases from 10 to 150 pixels along the $x$-direction. Consequently, the amplitude of $u_{y}$ also changes to insure a constant magnitude of the spatial derivative along $y$. The displacement corresponding to this strain distribution distribution serves as a modulation of the grid 


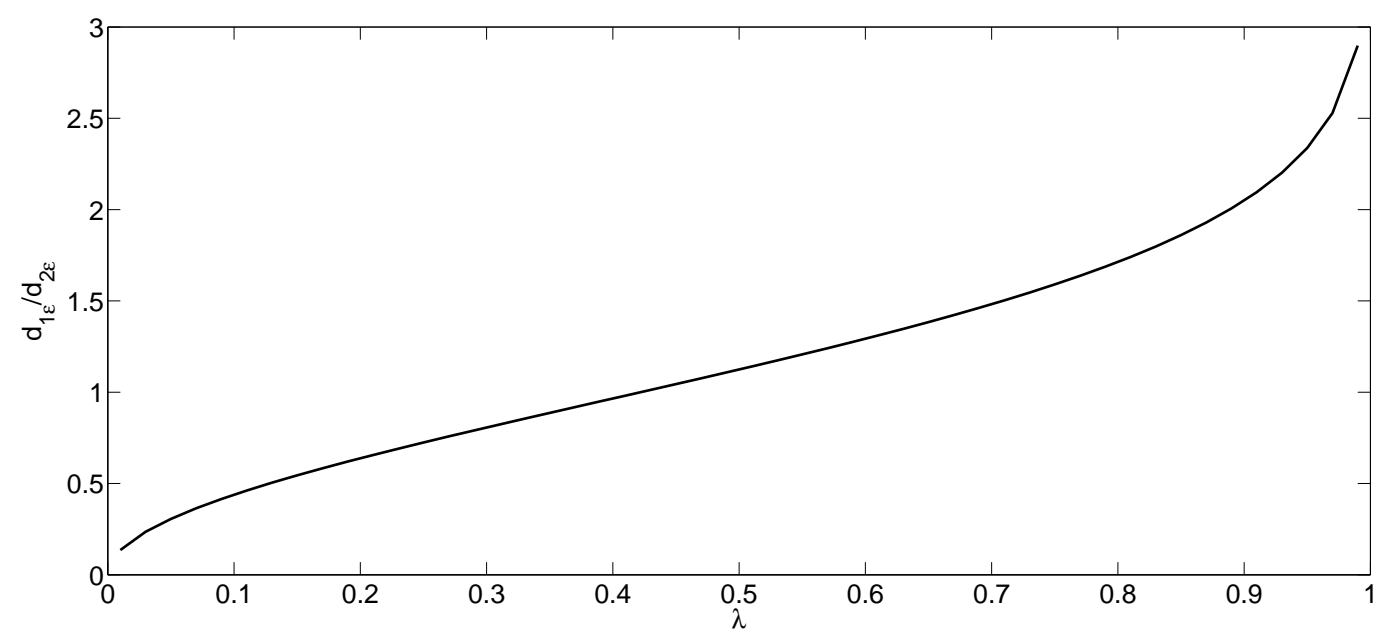

Figure 3: Ratio between $d_{1 \varepsilon}$ and $d_{2 \varepsilon}$ given by Equation 5.9. Note that $d_{1 \varepsilon}=d_{2 \varepsilon}$ for $\lambda=0.422$

model given in Equation 4.1 to build a synthetic grid image. The pitch $p$ of this grid is here equal to 5 pixels, which is a typical value chosen for encoding grids in practice. Figure 4-b shows the distribution estimated with the WFT, in which a Gaussian window has been employed. The standard deviation chosen here is the minimum for this quantity [171]: $\sigma=p=5$ pixels. It can be clearly seen that the estimated strain is smaller than the reference one, as also illustrated with the difference between the reference strain and the strain returned by the WFT (see Figure 4 $\mathrm{c}$ ). The relative bias $\lambda$ is deduced by dividing this loss in amplitude by the reference amplitude, thus the value at the cross-section of the distribution in Figure 4 4 c at $y=500$ pixels, by the reference amplitude. We give the obtained curve as a function of the period $\frac{1}{f^{\prime}}$ directly, this quantity being proportional to $x$. The bias is all the greater as the period is the smaller (on the left-hand side of the curve, near $\lambda=1$ ), as expected. The value for the relative bias obtained from this simulation (solid line) can be compared with that predicted with Equation 5.6 (dashed line). It can be checked that both curves are in very good agreement, illustrating the relevance of this simple predictive formula for the bias. Note that this monotonic evolution of the bias as a function of the period/frequency of the signal is not observed for some other types of windows which can be employed in the WFT, see [137].

\subsubsection{Link between measurement resolution and spatial resolution}

Even though it is possible to perform Monte-Carlo-based approaches to assess the measurement resolution in displacement and strain maps (see [172] for instance), it is of prime importance to have closed-form expressions for this measurement resolution to conveniently study the influence of various parameters. These expressions are obtained assuming that a Gaussian white noise affects the grid images (the standard deviation of this noise is a constant denoted $\sigma_{\text {image }}$ ). This is a rough assumption because $\sigma_{\text {image }}$ spatially varies and depends on the brightness in actual images, but we will in Section 6.5 how to tackle this issue. After pioneering work by Surrel in the discrete case and considering a triangular window in the WFT [130, a Gaussian analysis window was employed in [171] and the covariance matrix characterizing the noise in phase and phase derivative maps was pro- 


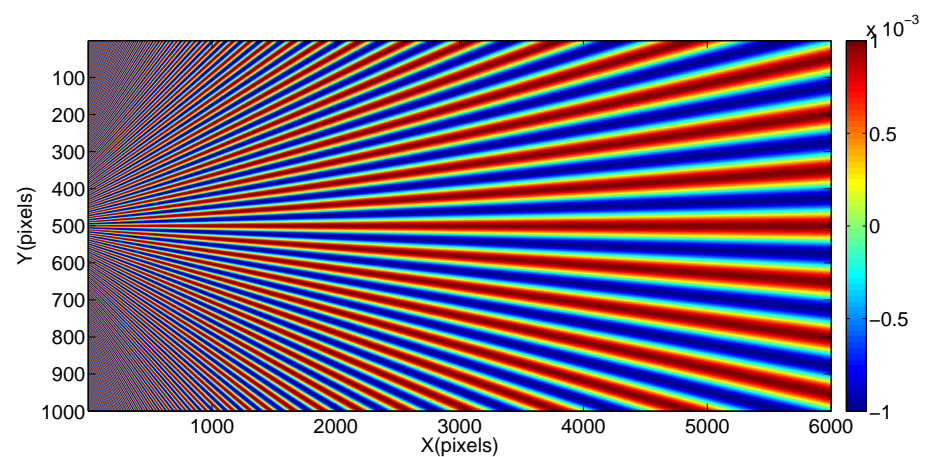

a- reference distribution

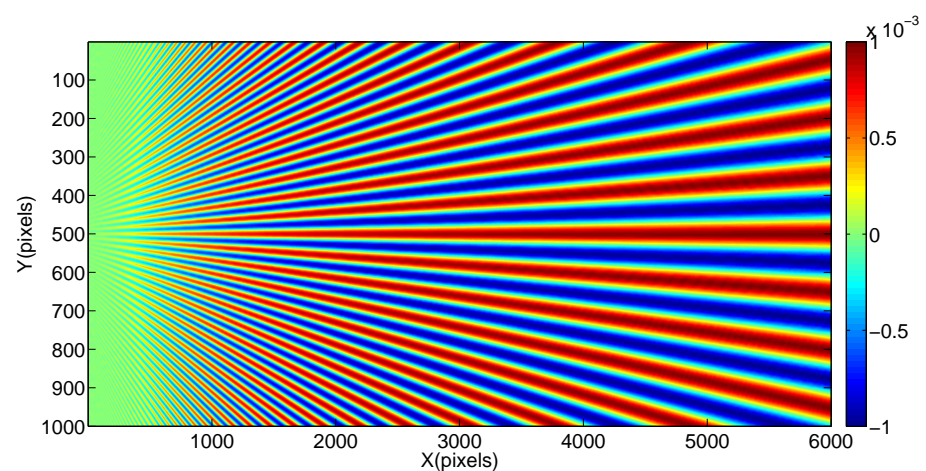

b- estimated distribution

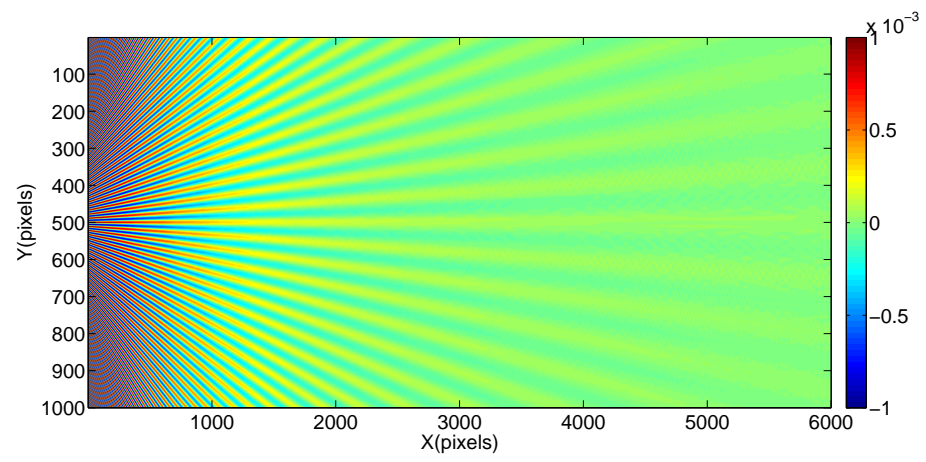

c- difference between reference and estimated distributions

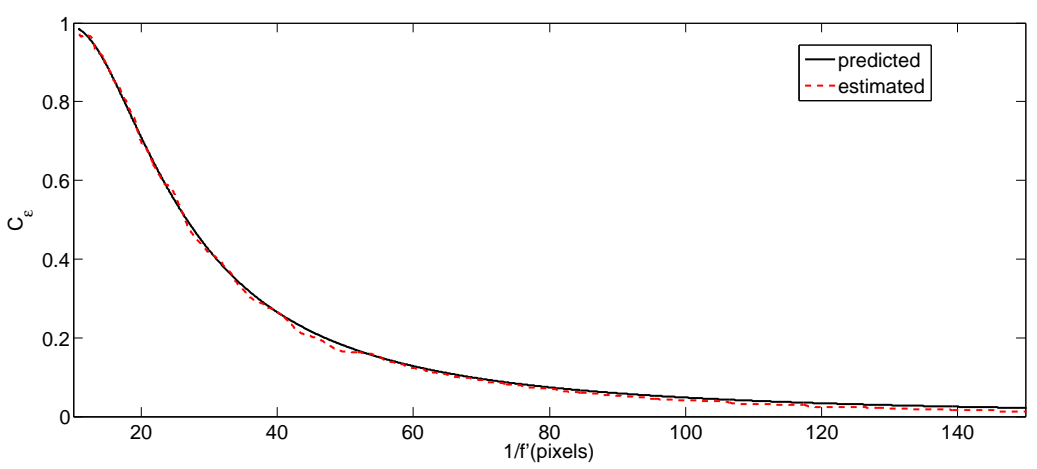

d- relative bias $\lambda$ as a function of the strain period

Figure 4: Illustration of the bias affecting the strain measurement. 
vided (results obtained with other types of windows are discussed in [137]). The noise variances in the phase and phase derivative maps are then easily deduced. Taking into account the link between changes in phases (resp. phase derivatives) on the one hand, and displacement (resp. strain) components on the other hand (see Equations 4.12 and 4.25), the following relationships were proposed in [78] between the measurement resolution and the first definition of the spatial resolution:

$$
\left\{\begin{array}{lll}
\sigma_{u} \times d_{1 u} & =p C_{u} \times \frac{\sqrt{\Delta_{x} \Delta_{y}}}{K} \times \sigma_{\text {image }} & \text { for displacement and } \\
\sigma_{\epsilon} \times d_{1 \epsilon}^{2} & =p C_{\epsilon} \times \frac{\sqrt{\Delta_{x} \Delta_{y}}}{K} \times \sigma_{\text {image }} & \text { for strain }
\end{array}\right.
$$

Note that Equation 5.10 b holds for normal strain components $\epsilon_{x x}$ and $\epsilon_{y y}$. For $\epsilon_{x y}$, the right-hand side term of Equation 5.10 should be divided by $\sqrt{2}$ (see Equation 4.16).

In these equations:

- $\sigma_{u}$ and $\sigma_{\epsilon}$ are respectively the standard deviation for the noise in the displacement and strain maps, so the corresponding measurement resolution according to the definitions adopted in Section 5.3.1

- $p$ is the pitch of the grid

- $C_{\varepsilon}$ and $C_{u}$ are coefficients depending solely on the nature of the window employed in the WFT. With a Gaussian analysis window, $C_{u}=\frac{3}{2 \pi^{\frac{3}{2}}}$ and $C_{\varepsilon}=\frac{9 \sqrt{2}}{2 \pi^{\frac{3}{2}}}$

- $\Delta_{x}$ and $\Delta_{y}$ represent here the dimensions of one pixel. Hence if all the dimensions are given in pixel, $\Delta_{x}=\Delta_{y}=1$

- $K$ is the modulus $|\Psi|$ of the WFT of the grid image. The moduli obtained for the WFT calculated along $x$ or $y$ are theoretically equal [171, so no distinction is made here in the notation for quantities calculated along $x$ and $y . K$ is theoretically equal to 78 ]

$$
K=\frac{\left|d_{1}\right| \gamma A}{2}
$$

where $\left|d_{1}\right|$ is the modulus of the coefficient of the first non-constant term in the Fourier expansion of the frng function which defines the profile of the grid lines in Equation 4.1 above, and $A$ and $\gamma$ are as defined in Equation 4.1 .

- $\sigma_{\text {image }}$ is the standard deviation of the noise impairing the grid images. In these equations, it is assumed to be constant for the sake of simplicity, but this assumption is not really satisfied in real grid images, as discussed in Section 6.5.

We obtain similar equations for the second definition of the spatial resolution by substituting $d_{1}$ by $d_{2} \frac{3 \sqrt{2}}{\pi} \sqrt{-\log (1-\lambda)}$ thanks to Equation 5.9 . This leads to

$$
\left\{\begin{aligned}
\sigma_{u} \times d_{2 u} & =\frac{p C_{u} \pi}{3 \sqrt{2} \sqrt{-\log (1-\lambda)}} \times \frac{\sqrt{\Delta_{x} \Delta_{y}}}{K} \times \sigma_{\text {image }} \text { for displacement and } \\
\sigma_{\epsilon} \times d_{2 \epsilon}^{2} & =-\frac{p C_{\epsilon} \pi^{2}}{18 \log (1-\lambda)} \times \frac{\sqrt{\Delta_{x} \Delta_{y}}}{K} \times \sigma_{\text {image }} \text { for strain }
\end{aligned}\right.
$$




\section{Measurement resolution}

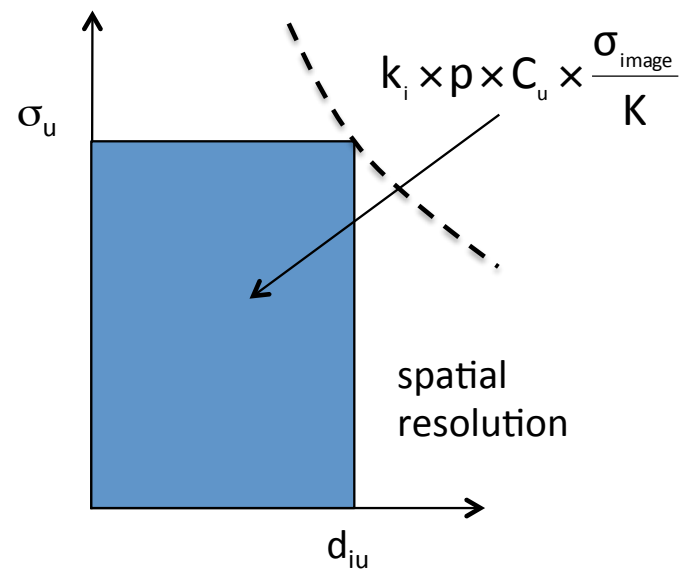

a- displacement resolution against spatial resolution

\section{Measurement \\ resolution}

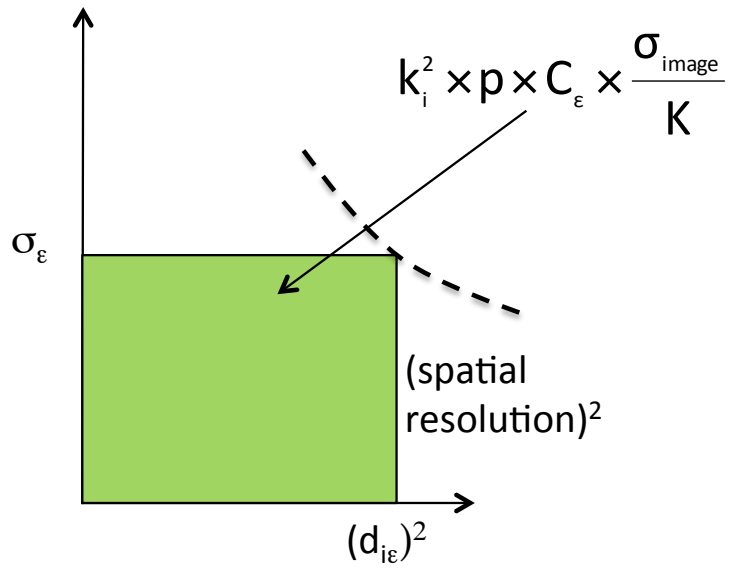

b- strain resolution against spatial resolution (squarred)

Figure 5: Graphical illustration of the link between displacement/strain resolutions and spatial resolution for a given homoscedastic noise in the grid image and modulus of the WFT $K . \quad k_{1}=1, k_{2}=\frac{\pi}{3 \sqrt{-2 \log (1-\lambda)}}, 0<\lambda<1$. Products $\sigma_{u} \times d_{1 u}$ and $\sigma_{\epsilon} \times d_{1 \epsilon}^{2}$, remain constant when $\sigma$ changes. Hence increasing $d_{1 j}, j=u, \epsilon$ causes $\sigma_{j}, i=u, \epsilon$ to decrease.

Equations 5.10 and 5.12 directly drive sensor noise propagation from grid images to displacement and strain maps.

Only one parameter can be changed by the user for a given set of grid images to be processed: $\sigma$ (or the width of the analysis window in the WFT). With the first definition of the spatial resolution, changing this parameter directly impacts the spatial resolution since $\sigma$ and spatial resolution are proportional in this case. Equations 5.10 tell us that both products $\sigma_{u} \times d_{1 u}$ and $\sigma_{\epsilon} \times d_{1 \epsilon}^{2}$ remain constant for a given noise impairing the grid images and for a given modulus $K$ of the WFT. This result is obtained whatever the frequency $f^{\prime}$ of the displacement/strain fields modulating the grid. This property is graphically illustrated in Figure 5 a for displacement and in Figure 5-b for strain. The area of both rectangles is proportional to the noise intensity in the grid images, and inversely proportional to the modulus of the WFT, thus to both the lighting intensity and the contrast of the grid images (see Equation 5.11). This is consistent with intuition. The area of both rectangles does not depend on $\sigma$ if $i=1$, thus does not depend on $d_{1 \epsilon}$ and $d_{1 u}$. It means that changing the spatial resolution by changing $\sigma$ only causes the upper right corner of both rectangles to slide along a curve defined by Equations 5.10 -a or -b, and the shape of these curves does not depend on $\sigma$.

With the second definition of the spatial resolution, the area of both rectangles depends on $\lambda$, which is now a parameters chosen the user. Changing only $\sigma$ and keeping $\lambda$ constant leads to the same conclusion as in the preceding case, but this is not the case anymore when $\lambda$ changes too. 


\subsubsection{D visualization of the link between strain resolution, spatial resolution and relative bias in the case of sinusoidal distributions}

Measurement resolution, spatial resolution and relative bias being interconnected through Equations 5.10, 5.12 and 5.6, it is difficult to have a clear view on the metrological performance of the measuring technique in terms of

- sensor noise propagation up to the final maps

- ability to distinguish close features in displacement and strain maps

- ability to provide the true value for the measurement (with the trueness as defined in [159).

As explained above, strain maps are much more affected by the bias than displacement maps, so we focus hereafter on strain maps only. Combining Equation 5.10 b for $d_{1 \varepsilon}$ (or Equation 5.12 -b for $d_{2 \varepsilon}$ ) and Equation 5.6 leads to the link between the strain resolution $\sigma_{\varepsilon}$, the spatial resolution $d_{1 \varepsilon}$ (or $d_{2 \varepsilon}$ ) and the relative bias $\lambda$. The user generally chooses the width of the by fixing $\sigma$, so for the first definition of the spatial resolution, $d_{1 \epsilon}$ is straightforwardly obtained as a multiple of $\sigma$, and the two other parameters are deduced from this choice. This is more subtle for $d_{2 \epsilon}$ since this latter quantity is driven by the bias $\lambda$ that the user must choose in addition to $\sigma$.

These two sets of links can be modeled each by a 3D plot in the strain resolution / spatial resolution / relative bias space. We propose here a synthetic example which relies on an ideal grid image, in which representative values for the various parameters involved in the calculations are considered. More precisely, it is obtained under the following conditions:

- We consider virtual and perfect grid images shot by a virtual 12-bit camera. Graylevel intensities are however encoded on 16 bits by multiplying the 12-bit camera output by $2^{4}$, in order to mimic the raw images given by popular cameras providing 16-bit image files. The whole dynamics of the bit depth is employed at any pixel, so $A=2^{15}$ and $\gamma=1$ in Equation 4.1 .

- The grid pitch $p$ is encoded with 5 pixels, which is a classic value for this parameter in practice. We explore values for $\sigma$ lying between the pitch $p$ of the grid (this is the minimum value according to [171]), so 5 pixels here, and twice this pitch, thus 10 pixels.

- The line profile is assumed to be an ideal pure sine. In this case, $d_{1}=0.5$ in Equation 5.11 [78]. In this example, with the values of the parameters given above, we have $K=2^{13}$.

- For $d_{1 \varepsilon}$, the synthetic phase distribution is a sine wave whose period $\frac{1}{f^{\prime}}$ lies between twice the grid pitch and 15 times the grid pitch (with values which are multiples of the pitch), thus $\frac{1}{f^{\prime}} \in[10,75]$ pixels. This pitch can be considered as a parameter and we can observe its influence on the bias.

- For $d_{2 \varepsilon}$, the bias evolves stepwise from 0.1 to 0.2 (we chose small values because users generally want a low bias in their measurements), so we have two parameters influencing the response, $\sigma$ which lies between 5 and 10 pixels, and $\lambda$ between 0.1 to 0.2 . 
- This value for the standard deviation is similar to that observed on average with the 16-bit image shot with the CCD camera used in Ref. [78]: $\sigma_{\text {image }}=350$ gray levels (see Section 6.5 to account for the actual response of a camera sensor, which is signal-dependent).

These parameters represent an ideal case: in real images, $\gamma$ is lower than one and $A$ lower than $2^{15}$. The line profile is not a pure sine function neither, so $d_{1}$ is lower than 0.5 (see the annex of Ref. 78 for further details). It means that the present simulation represents the upper bound of the metrological perfomance which can be reached with this aforementioned virtual camera.

For the first definition of the spatial resolution, Equation $5.10 \mathrm{~b}$ and 5.6 become respectively:

$$
\left\{\begin{array}{l}
\sigma_{\epsilon}=\left(5 \times\left(\frac{9 \sqrt{2}}{2 \pi^{3 / 2}}\right) \times \frac{1}{2^{13}} \times 350\right) \frac{1}{d_{1 \epsilon}^{2}} \simeq \frac{0.2441}{d_{1 \epsilon}^{2}} \\
\lambda \simeq e^{-0.5483 f^{\prime 2} d_{1 \epsilon}^{2}}, 10 \leq \frac{1}{f^{\prime}} \leq 75 \text { (in pixels) }
\end{array}\right.
$$

For the second definition, Equations 5.12 b and 5.6 give

$$
\left\{\begin{array}{l}
\sigma_{\epsilon} \simeq\left(-5 \times\left(\frac{9 \sqrt{2}}{2 \pi^{3 / 2}}\right) \times \frac{\pi^{2}}{18 \log (1-\lambda)} \times \frac{1}{2^{13}} \times 350\right) \frac{1}{d_{2 \epsilon}^{2}} \simeq \frac{-0.1339}{d_{2 \epsilon}^{2} \log (1-\lambda)} \\
d_{2 \epsilon}=\frac{\sqrt{2} \pi \sigma}{\sqrt{-\log (1-\lambda)}}, 5 \leq \sigma \leq 10 \text { (in pixels), } 0.1 \leq \lambda \leq 0.2
\end{array}\right.
$$

Both sets of equations represent each a 3D curve in the strain resolution-spatial resolutionrelative bias space, $f^{\prime}, \lambda$ and $\sigma$ being parameters. These two sets of curves are plotted in Figure 6 and Figure 7, respectively, along with their projections in the three planes of this space (see subfigures -b, -c and -d). The following comments can be drawn:

Figure 6;

- in Figure 6-a and 6-b, it is clear that for a given spatial resolution, the bias increases as the frequency of the signal increases

- it is clearly visible in Figure 6-a that the strain resolution/spatial resolution curves do not depend on the period of the sine wave which serves as a reference, thus on the bias. This is confirmed by the top view shown in Figure 6rc

- from a qualitative point of view and as expected, the strain resolution decreases and the relative bias increases as the spatial resolution increases, see Figure 6 b b and Figure 6rd, respectively

Figure 7

- all the three parameters are now interconnected, since the strain resolution/spatial resolution curves now depend on the bias, even if the effect is slight for this set of parameter values, as can be seen in Figure 7 -c

- fixing the value of $\lambda$ and changing the value of $\sigma$ leads to a curve. The set of curves obtained for various values of $\lambda$ is not plotted along the same vertical direction, which was the case when considering $d_{1}$ instead of $d_{2}$ 


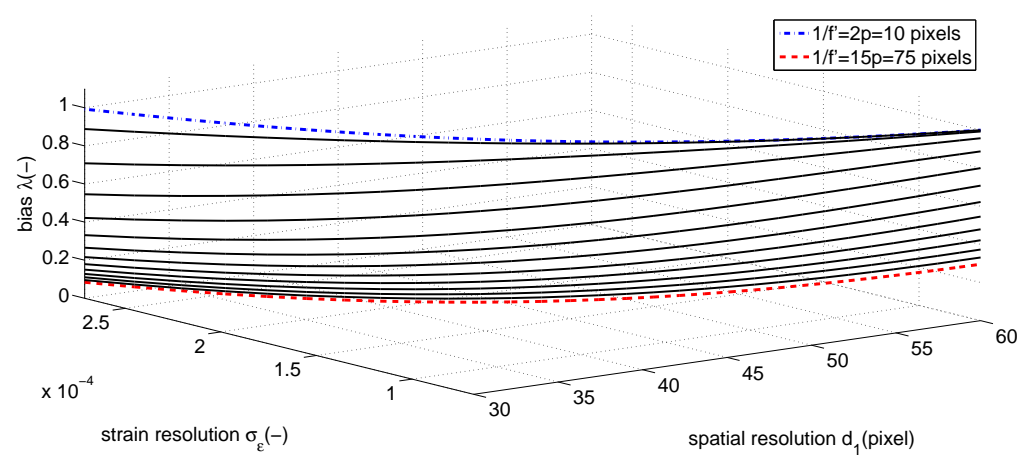

a- link between spatial resolution, strain resolution and relative bias

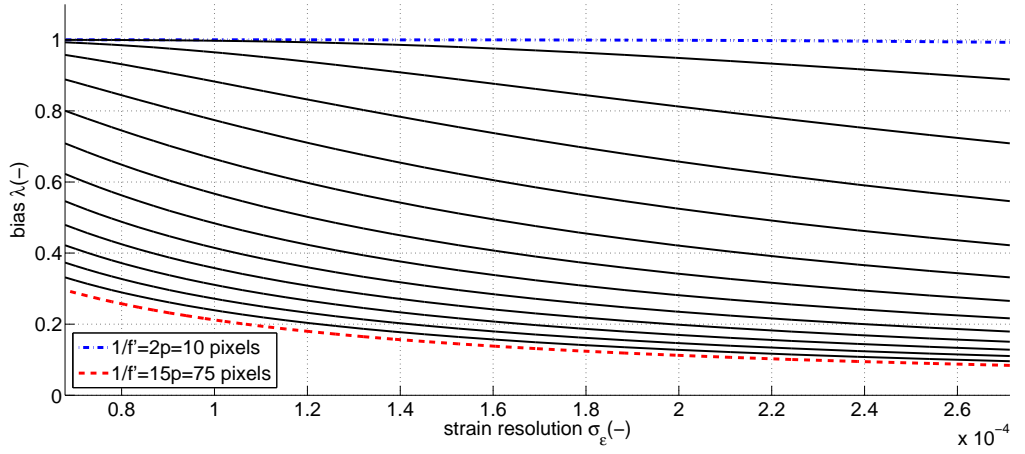

b- relative bias vs. strain resolution

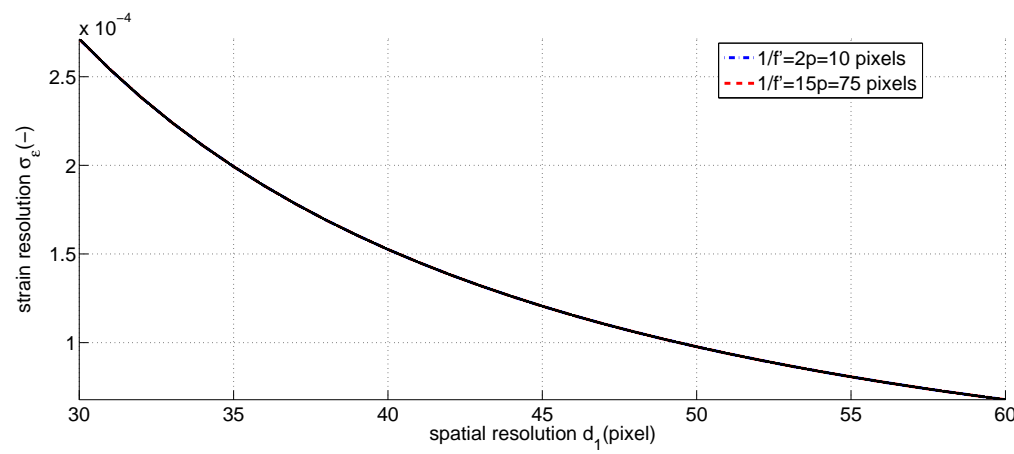

c- strain resolution vs. spatial resolution

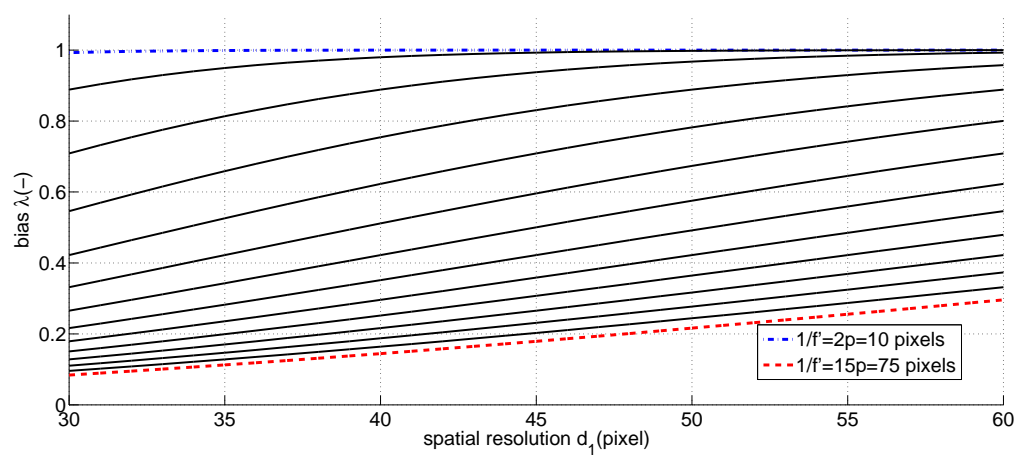

d- relative bias vs. spatial resolution

Figure 6: Link between relative bias, spatial resolution and strain resolution. The relative bias depends of the measurand itself. The measurand is here a strain field modeled with a sine wave of period $\frac{1}{f^{\prime}}$. $\frac{1}{f^{\prime}}$ increases stepwise from $\frac{1}{f^{\prime}}=2 p=10$ pixels to $\frac{1}{f^{\prime}}=15 p=$ 75 pixels (step equal to $p$ ). 


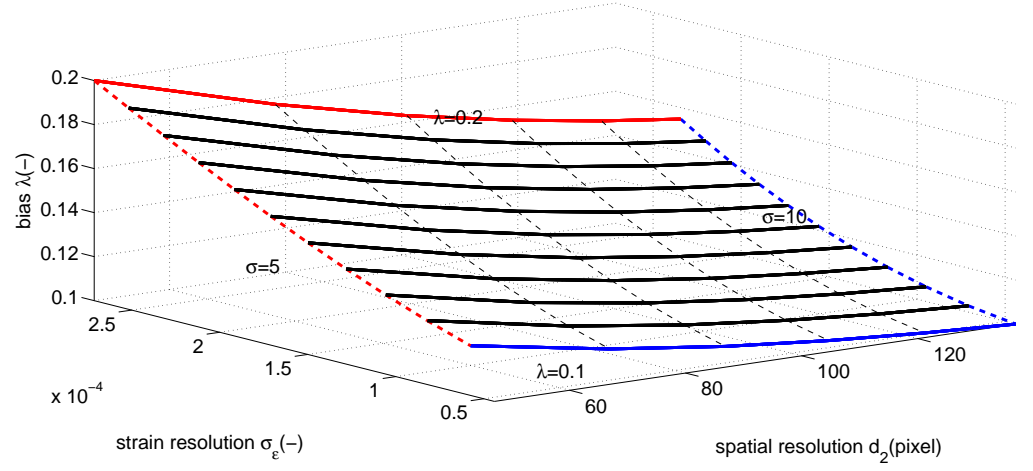

a- link between spatial resolution, strain resolution and relative bias

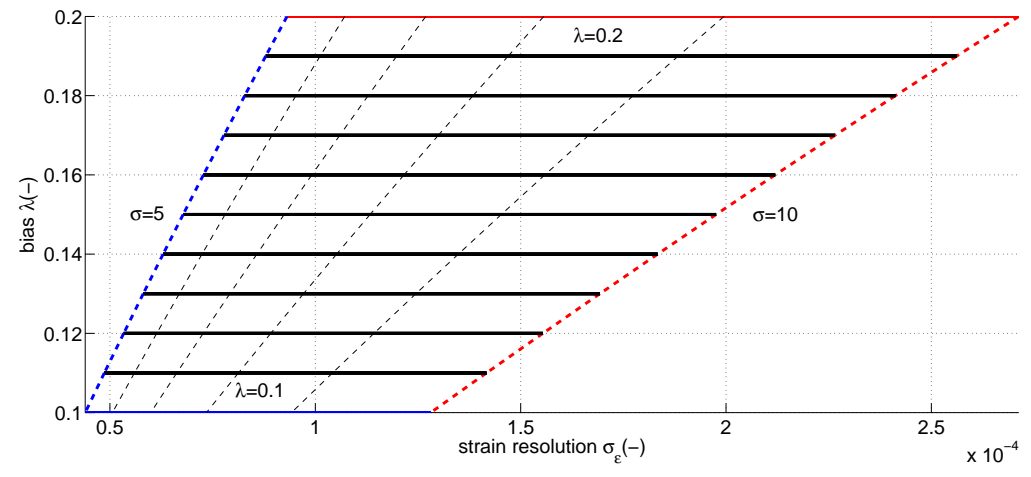

b- relative bias vs. strain resolution

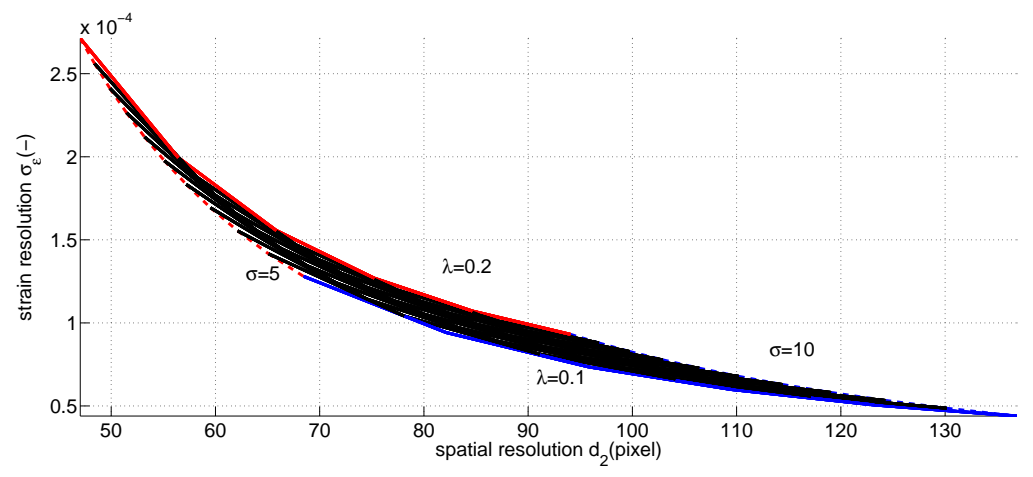

c- strain resolution vs. spatial resolution

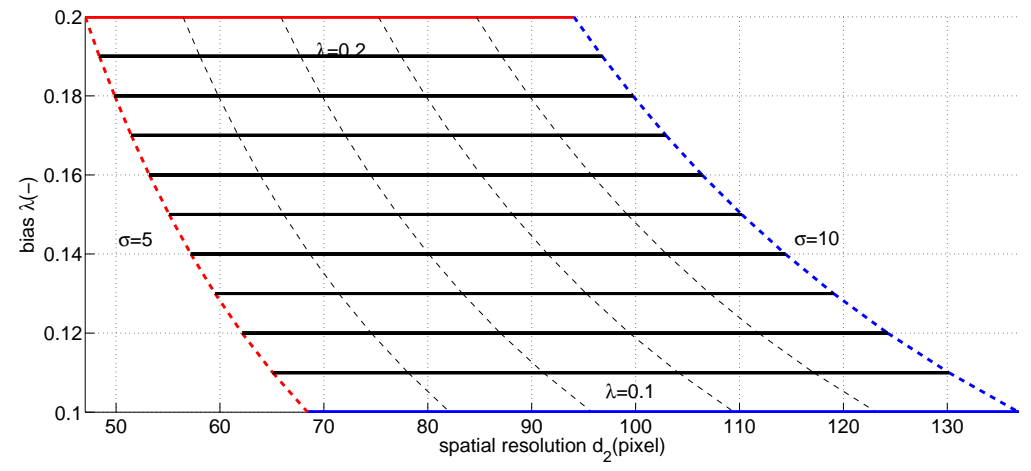

d- relative bias vs. spatial resolution

Figure 7: Link between spatial resolution $d_{2 \epsilon}$, strain resolution $\sigma_{\epsilon}$ and relative bias $(\lambda)$. The bias $\lambda$ is in this case a parameter chosen by the user. It lies here between 0.1 ad 0.2 . 
- the domain of definition of the strain resolution/spatial resolution equation now depends on the bias.

- since we considered low values for the bias in this example $(0.1<\lambda<0.2)$, we can see that $d_{2 \varepsilon}>d_{1 \varepsilon}$, as expected when considering the ratio between these two quantities shown in Figure 3

- for this set of parameter values, the spatial distribution reaches greater values than in the preceding case while the strain resolution spans the same interval. This illustrates on this simple example that the metrological properties must be given with a clear definition of the parameters involved.

\subsubsection{Case of real distributions: towards deconvolution of strain maps}

A wide range of frequencies is involved in real displacement and strain maps. The estimate for $\lambda$ discussed in the particular case of a sine function can be employed here to see how real strain maps are corrupted by the bias. According to Equation 4.26, the FT of any sought noise-free in-plane strain distribution $\widetilde{\varepsilon}_{k l}, k, l \in x, y$ is defined by

$$
\widehat{\widetilde{\varepsilon}_{k l}}(\xi, \eta)=\int_{-\infty}^{+\infty} \int_{-\infty}^{+\infty} \widetilde{\varepsilon}_{k l}(x, y) e^{-2 i \pi(\xi x+\eta y)} \mathrm{d} x \mathrm{~d} y
$$

and the IFT of $\widehat{\varepsilon_{k l}}$ (according to Equation 4.28)

$$
\widetilde{\varepsilon}_{k l}(x, y)=\int_{-\infty}^{+\infty} \int_{-\infty}^{+\infty} \widehat{\widetilde{\varepsilon}_{k l}}(\xi, \eta) e^{2 i \pi(\xi x+\eta y)} \mathrm{d} \xi \mathrm{d} \eta
$$

The discrete FT would be employed in practice to calculate these quantities, but we use here the continuous approach to be consistent with the results discussed in Section 4.4 . According to Equation 5.5, we know that after applying the LSA with a Gaussian analysis window of standard deviation $\sigma$, the amplitude of any sine wave of frequency $f^{\prime}$ is multiplied by a factor equal to $e^{-2 \pi^{2} \sigma^{2} f^{\prime 2}}$ if a Gaussian window is employed in the WFT. The Fourier transform being linear, each term in the integral defining the inverse Fourier transform of $\widehat{\widetilde{\varepsilon}}_{k l}$ is affected by a weighting factor lower than one, and whose amplitude depends on the frequency of the harmonic. Consequently, the sought strain field $\widetilde{\varepsilon}_{k l}$ impaired by the bias writes as follows

$$
\widetilde{\varepsilon}_{k l}(x, y)+b_{k l}(x, y)=\int_{-\infty}^{+\infty} \int_{-\infty}^{+\infty} \widehat{\widetilde{\varepsilon}_{k l}}(\xi, \eta) e^{2 i \pi(\xi x+\eta y)} e^{\left(-2 \pi^{2} \sigma^{2}\left(\xi^{2}+\eta^{2}\right)\right)} \mathrm{d} \xi \mathrm{d} \eta
$$

So the bias affecting the strain field $\varepsilon_{k l}$ is equal to

$$
b_{k l}(x, y)=\int_{-\infty}^{+\infty} \int_{-\infty}^{+\infty} \widehat{\widetilde{\varepsilon}_{k l}}(\xi, \eta) e^{2 i \pi(\xi x+\eta y)}\left(1-e^{\left(-2 \pi^{2} \sigma^{2}\left(\xi^{2}+\eta^{2}\right)\right)}\right) \mathrm{d} \xi \mathrm{d} \eta
$$

We can see that the bias is not constant throughout a strain map since it depends on its local "frequential signature". It is all the greater as the coefficients of the highest frequencies involved in the Fourier transform of the sought noise-free strain map are high. Thus, local refined details in strain maps are impaired since they need high frequencies to be correctly described. The consequence of this phenomenon is that details are blurred.

It is worth mentioning that this weighting function $e^{\left(-2 \pi^{2} \sigma^{2}\left(\xi^{2}+\eta^{2}\right)\right)}$ rapidly decreases to zero as the frequencies along $x$ and $y$ increase because of its exponential nature. For 


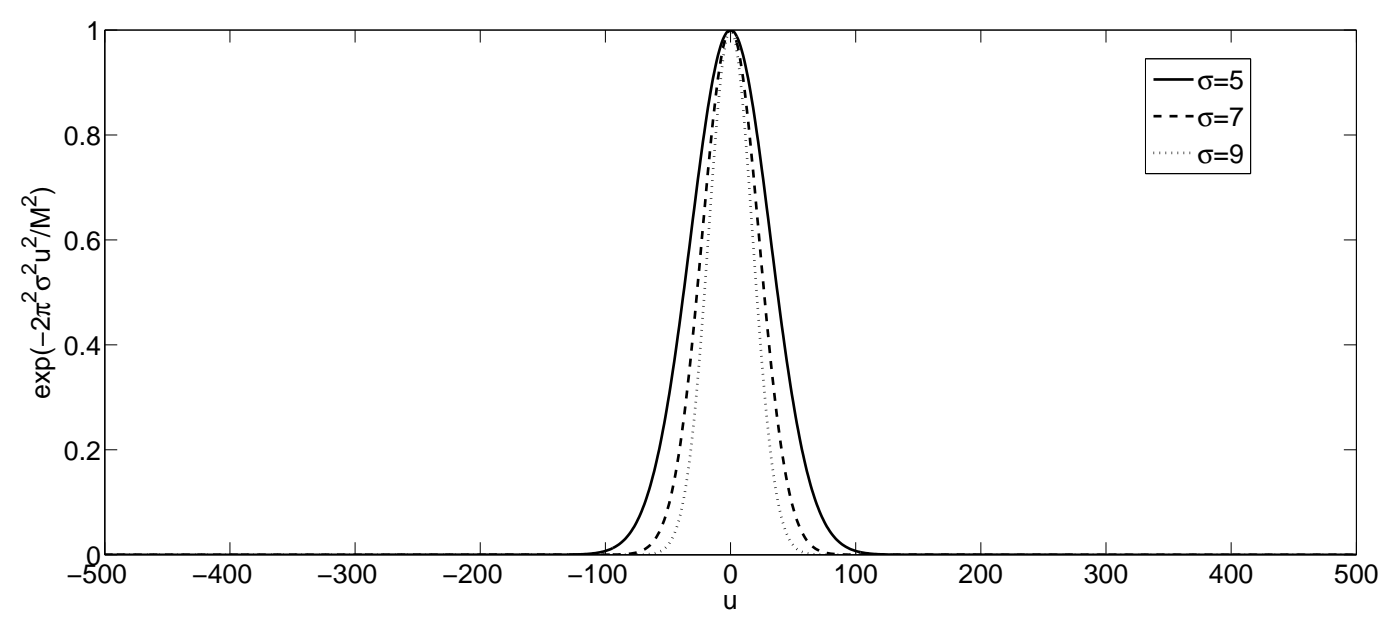

Figure 8: Cross-section along the $u$-axis of the weighting function affecting the Fourier coefficients. $M=500$ pixels.

instance, considering the cross-section along the $\xi$-axis of this function defined in the amplitude spectrum leads to the curve shown in Figure 8 , in the particular case $M=$ 1001 pixels (frequencies $\xi$ and $\eta$ lie between 0 and 0.5 cycle per period in the discrete case). Three different values for $\sigma$ are considered, lying from $\sigma=5$, which is a common value for the standard deviation of the Gaussian analysis window, to $\sigma=9$. It can be observed that most of the coefficients are multiplied by a factor close to zero, thus nullifying their influence in the measured strain map and causing blur to appear. Choosing a narrower analysis window such as a triangular one would lead to a wider weighting function, but the strain resolution would be impaired, in addition to some specific problems due to such an analysis window (for instance, the need for an integer value of pixels for the width of the window) [137.

Another important conclusion is that assessing the bias for a given strain map is not possible since we measure in practice a strain map $\varepsilon_{k l}$ which is biased and noisy, and not the actual, noise-free and unbiased sought distribution $\widetilde{\varepsilon}_{k l}$. Consequently, dividing naively the Fourier coefficients of the measured strain distribution by the weighting function $e^{\left(-2 \pi^{2} \sigma^{2}\left(\xi^{2}+\eta^{2}\right)\right)}$ to retrieve the Fourier coefficients of the actual strain distribution is completely unstable. The reason is that away from the center of the amplitude spectrum, we divide tiny quantities, namely Fourier coefficients mainly due to measurement noise, by coefficients which are also very close to zero. Retrieving the unbiased distribution from the measured one is in fact equivalent to deconvolve noisy strain maps. Deconvolution in the presence of noise, also called restoration, is however a problem as such in the image processing community. The reason is that as explained above, the problem becomes ill-posed in the sense of Hadamard [173], meaning that there is no unique solution. As a general remark, solving this type of problem requires suitable strategies based on regularization, and/or the introduction of a priori knowledge on noise or on the sought quantity. A broad literature on image restoration is available in the image processing community (see [174] for the general case or Ref. [175] for instance in the representative field of astronomy). Nevertheless, the main conclusion of an exploratory work on deconvolution of strain maps published in [169] is that restoration tools suitable for strain maps still remain to be invented, though we are here in the favorable case of non-blind deconvolution. It means that the so-called Point Spread Function (i.e. the function causing the blur by convolution of 


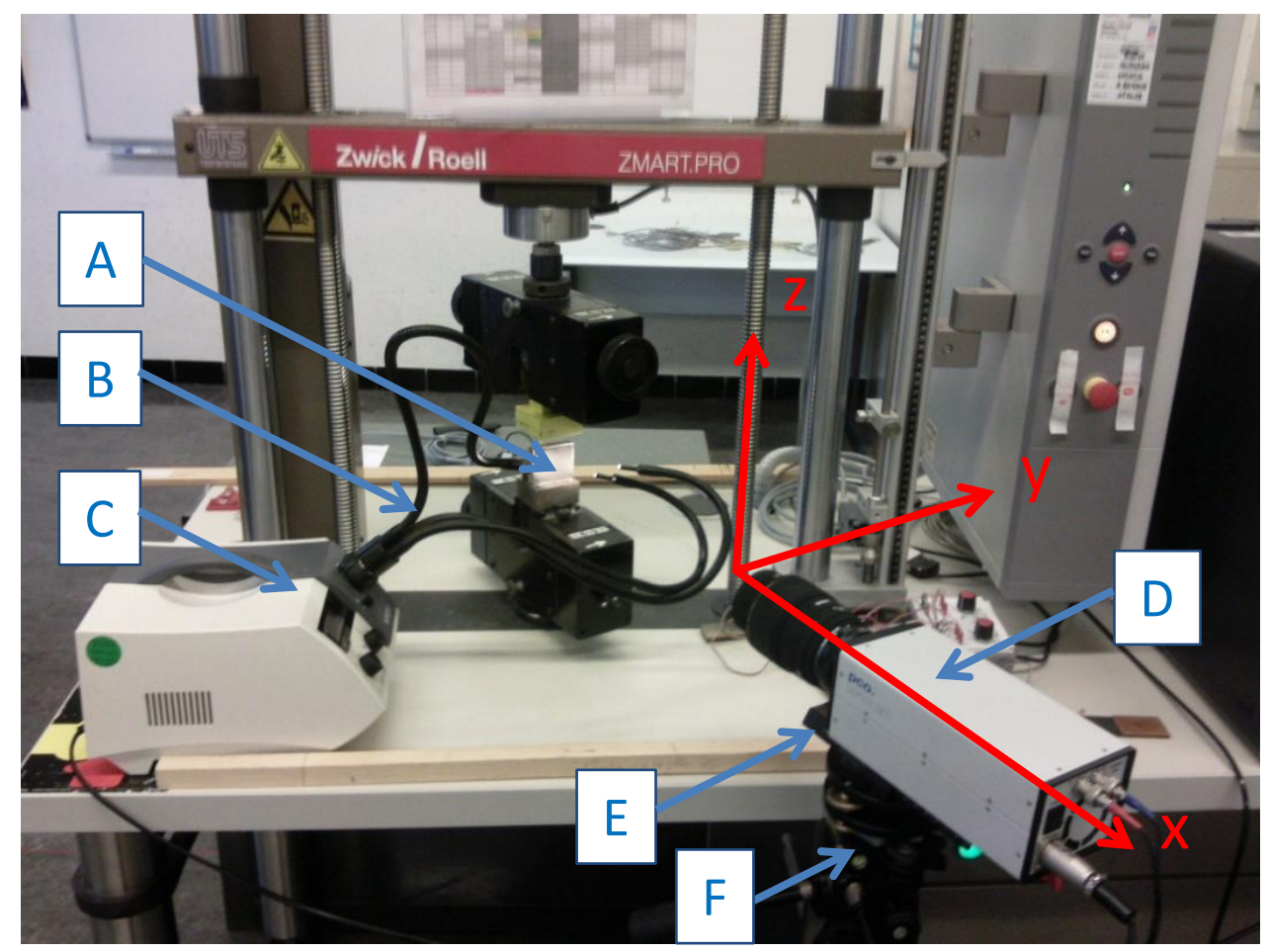

Figure 9: Typical experimental setup. A: camera, B: lighting, C: direct current transformer, D: digital camera, E: two translation stages, F: three-way photographic head.

the actual map) is known a priori.

\subsubsection{Conclusion on the metrological performance}

In conclusion, the fact that measurements are impaired by systematic (bias) and random errors shall not be considered as crippling for an effective use of the grid method for inplane displacement and strain measurement: even common electrical strain gauges also feature such a drawback like any other measuring tool. The positive point here is that some information is available to quantify the main metrological parameters characterizing the grid method when the WFT is used to process grid images.

\section{Some aspects on the practical implementation of the grid method}

We address here briefly some practical aspects of the grid method. This can turn out to be potentially useful for the reader interested in applying this technique.

\subsection{Setting up the grid method}

Figure 9 shows a typical experiment carried out with the grid method (here a compression test). A grid was deposited on the front face of the specimen beforehand (see Section 3 above). In this experiment, the specimen (A) is placed in a conventional tensile machine. Its front face is illuminated with a steady light (B) to avoid any light flickering. This light 
is supplied by a direct current transformer (C). A digital camera (D) is placed in front of the specimen to capture grid images while applying the load.

Adjusting the location of the camera must be made carefully to have the camera sensor as parallel as possible with the front face of the specimen. The camera is therefore mounted first on two perpendicular translation stages (E) which allows to adjust the location of the camera in the $x-y$ horizontal plane. It is also possible to move the camera along the $z$ direction with the column mechanism of the tripod. A rotation about each of the three perpendicular axes (front tilt, side tilt and panoramic) is also possible with a suitable three-way ball head (F), each rotation being adjusted with a separate lever. These six degrees of freedom permit framing adjustment to have the camera sensor as parallel as possible with the front face of the specimen.

With a fixed focal lens, the distance between front face of the specimen and camera influences the number of pixels used to encode one grid pitch. This number must be as close as possible to an integer when using some types of windows in the WFT [137]. Even when this is not a strict requirement, this condition should be satisfied with some cameras whatever the choice of the window. The orientation of the camera is indeed often adjusted in such a way that the lines of the grid are aligned with the lines and columns of the camera sensor. In this case, non-integer encoding increases the risk of parasitic fringes in strain maps due to some slight moiré effect between grid and camera sensor (see Section 6.7), especially for small strain values. Depositing the grid in such a way that the lines are inclined with respect to the lines and columns of the camera sensor strongly contributes to avoid this undesirable phenomenon. This point is discussed in depth in [176], where a strategy for finding an optimum angle is proposed. Defocusing also limits this phenomenon by smoothing out the high frequency components of the image and enforcing the Nyquist sampling condition. This however induces the contrast in images to decrease, thus the modulus $K$ of the WFT to decrease too, which causes the noise level in final strain maps to increase according to Equation $5.10,5.12$,

At the end and regardless of issues caused by distortion which may occur (see Section 6.4), the number of pixels per grid period can be checked in various zones of the grid images. Possible differences help the user rotate the camera in the right direction.

\subsection{Extrinsic and intrinsic parameters, or how to reduce sensor noise propagation into strain maps?}

The user is generally interested in improving the quality of the maps he obtains. It means that the noise impairing the maps which is due to camera sensor noise propagation must be as small as possible, especially when small strains are measured. To examine which setting must be adopted, it is interesting to examine in turn the influence of each quantity involved in Equations 5.10 and 5.12. This will help the user understand what can be done in practice to influence the metrological performance in the right direction. Again, we only consider the second equation which concerns strain. However similar conclusions can be drawn for displacement.

We propose to rewrite Equations $5.10 \mathrm{~b}$ and 5.12 -b to distinguish intrinsic and extrinsic parameters. The first ones represent the signature of the technique itself. They also depend on the settings which are chosen and mastered by the user, within certain limits. The second ones mainly depend on external factors, on which the user has only a limited influence, if not no influence at all. In this spirit, Equation $5.10 \mathrm{~b}$ and $5.12 \mathrm{~b}$ can be 
rewritten as follows:

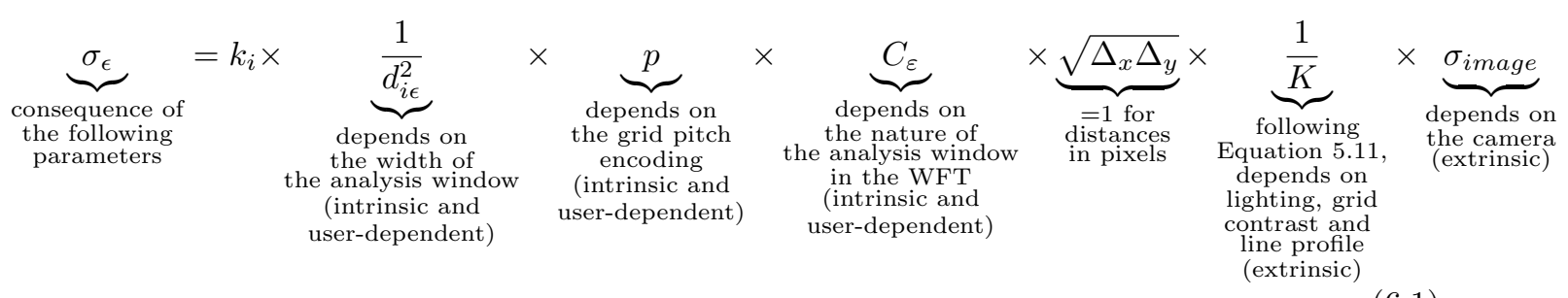

where $i=1$ for the first definition of the spatial resolution, and $i=2$ for the second definition. $k_{1}=1$ and $k_{2}=\frac{\pi}{3 \sqrt{-2 \log (1-\lambda)}}, 0<\lambda<1$. The following comment can be drawn on the different parameters:

- $d_{1 \epsilon}$ : The spatial resolution is fixed by the user as soon as he chooses the width of the analysis window employed in the WFT, since both quantities are equal for this definition of the spatial resolution.

- $p$ : The number of pixels per grid period is also a parameter chosen by the user. For some types of analysis windows, it is generally recommended to have an integer number for this quantity [137].

- $C_{\varepsilon}, C_{u}$ : These coefficients solely depend on the type of analysis window employed in the WFT (see [137] for other types of windows). Obviously, the lower the values for $C_{\epsilon}$ and $C_{u}$, the lower the noise level due to sensor noise in the final maps.

- $\frac{1}{K}: K$ is the modulus of the WFT. It is calculated at any pixel. Assuming a perfect surface marking and according to the grid model in Equation 4.1, this quantity is defined in Equation 5.11. From the definition of $K$ in Equation 5.11, we can conclude that the higher amplitude $A$ and contrast $\gamma$, the lower the value for $K$ and thus the lower the noise level caused by sensor noise in the final maps, which is in accordance with intuition. The highest value for $\left|d_{1}\right|$ is obtained with a sine profile: we have $d_{1}=0.5$ in this case. Any other profile would lead to a lower value. In practice, the user has a limited influence on these parameters: he can adjust light intensity (thus adjust $A$ ), but this intensity is often heterogeneous over the surface of the grid. Note that this phenomenon has no real influence on the phase extracted by the WFT as long as the brightness does not significantly evolves over the surface of the window employed in the WFT. In the same way, the line profile depends on the quality of the surface marking, and thus mainly on the quality of the grid which is transferred or deposited.

- $\sigma_{\text {image }}$ is the equivalent standard deviation of the noise impairing grid images shot with a CCD camera [78. It is assumed here to be constant, but the actual value is a function of pixel intensity, as discussed in Section 6.5.

\subsection{Noise pattern impairing retrieved maps, spatially correlated noise}

It is worth mentioning that noise in displacement and strain maps is spatially correlated. It has been shown in Ref. [171] that in phase maps, this spatial correlation manifests itself by "blobs" covering several pixels instead of the grainy aspect that would give a noise impairing the grey level at each pixel independently. This point is also discussed in [177. This is the consequence of the fact that noise affecting displacement and strain maps 
is spatially correlated. The phenomenon can be assessed with the correlation function between the noise at two points $M_{1}$ and $M_{2}$ in a map. This quantity writes as follows:

$$
\operatorname{Cor}\left(e_{i j}^{k}\left(M_{1}\right), e_{i j}^{k}\left(M_{2}\right)\right)=\frac{\operatorname{Covar}\left(e_{i j}^{k}\left(M_{1}\right), e_{i j}^{k}\left(M_{2}\right)\right)}{\sqrt{\operatorname{Var}\left(e_{i j}^{k}\left(M_{1}\right)\right) \operatorname{Var}\left(e_{i j}^{k}\left(M_{2}\right)\right)}} \quad i, j \in\{x, y\}, k \in\{u, \varepsilon\}
$$

The whole covariance matrix for the phase and phase derivative in grid images affected by homoscedastic noise has been established in [171] when a Gaussian analysis window is used in the WFT. Results obtained with other analysis windows are discussed in [137. Displacement and strain components being linked through Equations 4.14 and 4.18 with the phase and the phase derivatives, respectively, we can deduce the correlation function for these quantities. From the components of the covariance matrices given in [171] and denoting $s=x_{2}-x_{1}$ and $t=y_{2}-y_{1}$, this leads to:

for displacement:

$$
\operatorname{Cor}\left(e_{i}^{u}\left(M_{1}\right), e_{i}^{u}\left(M_{2}\right)\right)=e^{\frac{-\left(s^{2}+t^{2}\right)}{4 \sigma^{2}}} \quad i, j \in\{x, y\}
$$

for strain:

$$
\begin{aligned}
& \operatorname{Cor}\left(e_{i j}^{\varepsilon}\left(M_{1}\right), e_{i j}^{\varepsilon}\left(M_{2}\right)\right)=\frac{1}{2}\left(2-a_{1} \frac{s^{2}}{\sigma^{2}}-a_{2} \frac{t^{2}}{\sigma^{2}}\right) e^{\frac{-\left(s^{2}+t^{2}\right)}{4 \sigma^{2}}} \\
& \text { with }\left\{\begin{array}{l}
\left(a_{1}, a_{2}\right)=(1,1) \text { if }(i, j)=(x, y) \\
\left(a_{1}, a_{2}\right)=(2,0) \text { if }(i, j)=(x, x) \\
\left(a_{1}, a_{2}\right)=(0,2) \text { if }(i, j)=(y, y)
\end{array}\right.
\end{aligned}
$$

The second equation is valid only if no additional filtering of the displacement maps is performed before differentiation to get the strain components. Interestingly, the correlation between the noise in displacement maps at two different points is a Gaussian function of the distance between $M_{1}$ and $M_{2}$. Its standard deviation is $\sqrt{2} \sigma$ (instead of $\sigma$ for the standard deviation of the Gaussian window used in the WFT to extract phases). If we adopt the classic $3-\sigma$ rule to assess the width of a Gaussian window, it means that beyond $6 \sqrt{2} \sigma$ in distance, points in displacement maps are nearly not correlated. The 2D Gaussian being isotropic, the global aspect of blobs in displacement maps is isotropic. This property is illustrated in Figure 10 a, where a circle featuring a diameter equal to $6 \sqrt{2} \sigma$ has been superposed on blobs observed in a typical displacement map. As can be seen, this assessment is in agreement with what is observed.

For strain maps, the conclusion is slightly different: the same Gaussian function as above is multiplied by a quadratic function. If $i \neq j$, the quadratic function is isotropic, thus leading the blobs to be isotropic on average. The Gaussian distribution being however multiplied by a function which is lower than one, the average size of the blobs is reduced in an isotropic manner. It means than the size of the blobs becomes lower than that of the blobs impairing the displacement maps, the latter being governed by the Gaussian function alone. This is clearly visible in Figure 10 b, where the blobs for $\epsilon_{x y}$ are smaller than in Figure 10-a for $u_{x}$. If $i=j=x$ (resp. $=y$ ) for instance, this function only depends on the coordinate along direction $x$ (resp. $y$ ), namely $s$ (resp. $t$ ). This function being lower than one, this leads the isotropic Gaussian distribution to be shrunk along direction $x$ (resp. $y$ ), the other dimension being unchanged. This is exactly what is observed in Figures 10-c (resp. Figure 10-d) where the blobs are clearly shrunk along $x$ (resp. y). Figures 10-e 


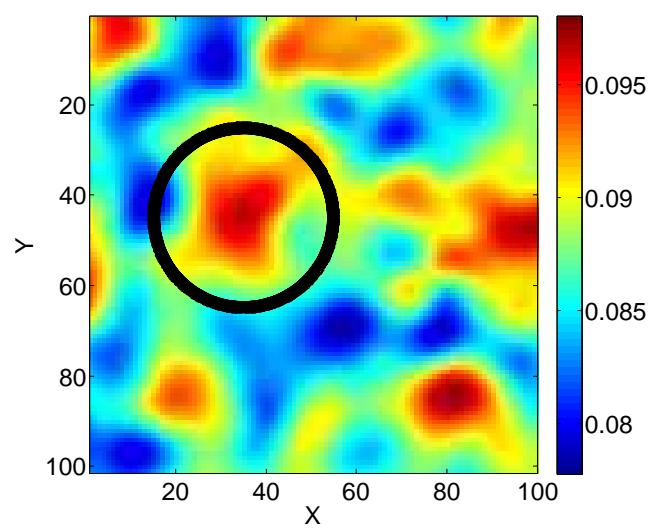

a-

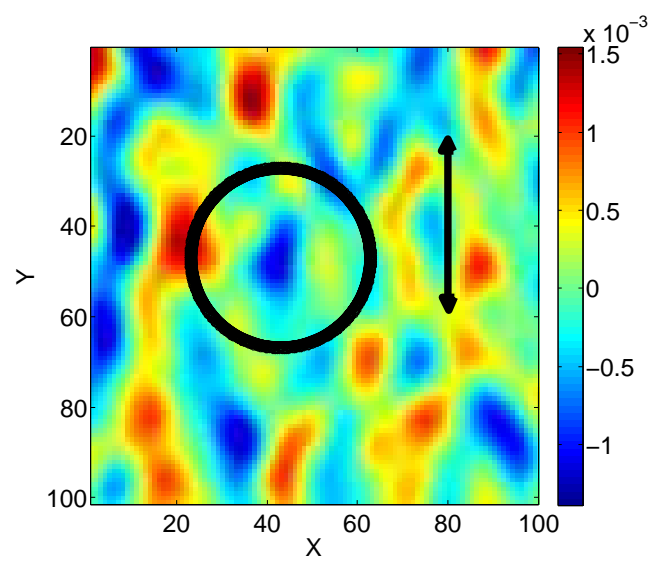

c-

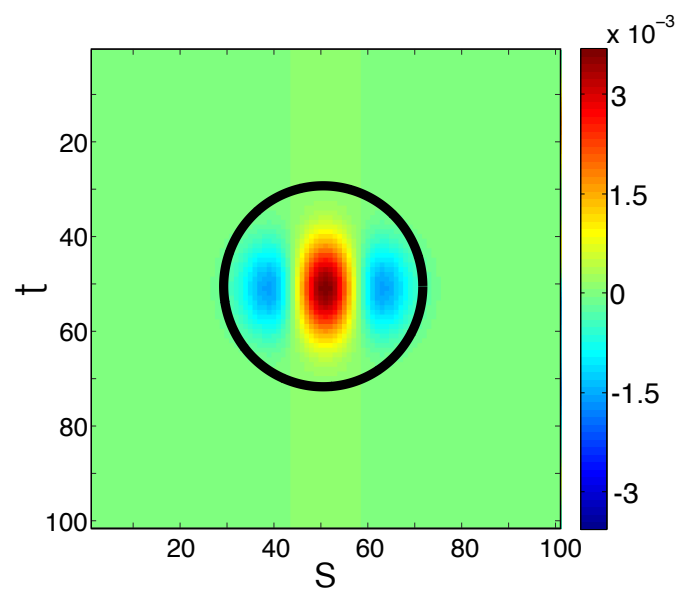

e-

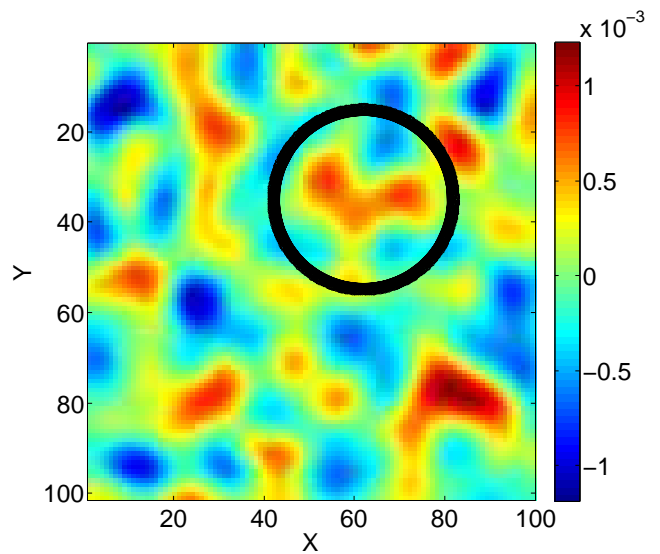

b-

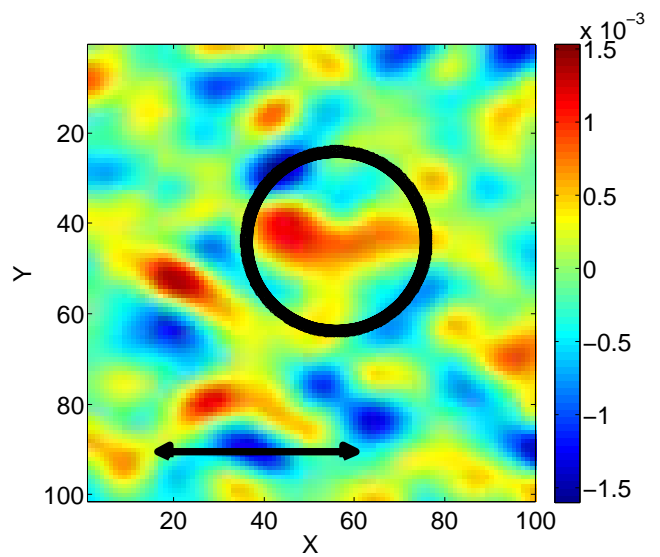

d-

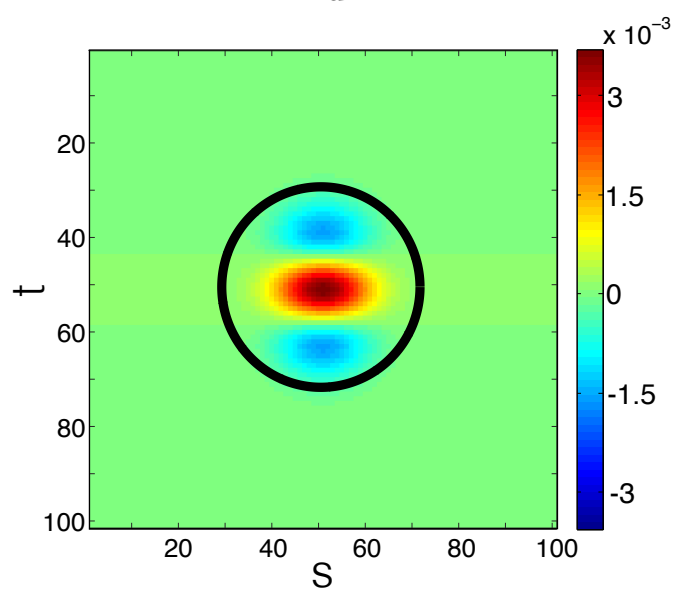

f-

Figure 10: Close-ups of typical examples of blob distributions in experimental displacement and strain maps measured during a translation test. a- blobs in typical displacement maps are isotropic on average (here $u_{x}$ as an example). They are within a circle of diameter $6 \sqrt{2} \sigma$. b- blobs in $\epsilon_{x y}$ shear strain maps are isotropic on average. Their average dimension is smaller than those found for the displacement map (to be compared with the size of the blobs in subfigure a-). c- blobs in $\epsilon_{x x}$ strain maps are shrunk along direction $x$ d- blobs in $\epsilon_{y y}$ strain maps are shrunk along direction $y$. e- correlation function for the random noise process in $\epsilon_{x x}$. f- correlation function for the random noise process in $\epsilon_{y y}$. All dimensions in pixels, 1 pixel represents $40 \mu \mathrm{m}$ on the specimen for subfigures a-, b-, c-, d-. 
and 10 -f represent the correlation function for the random noise process in $\epsilon_{x x}$ and $\epsilon_{y y}$, respectively (see Equation 6.4 for the corresponding analytical expression). It is clear that in terms of shape and size, the corresponding dots are in agreement with those depicted in Figures 10-c and 10-d. This observation is expected on average, the experimental process observed here being non-deterministic.

As a final remark, we can note that it would be useful to have a practical decision rule to discriminate between noise blobs and actual details of a strain field. Interestingly, a similar problem arises in positron emission tomography (PET) an functional magnetic resonance imaging (FMRI), also affected by a correlated noise (see for instance [178]). Under white noise assumption, simple hypothesis testing with Bonferroni correction gives a detection threshold with a controlled error rate. This approach is, however, too conservative in the presence of a correlated noise. Instead, approaches based on the random field theory have been developed [179, 180]. Similar techniques for displacement and strain fields have still to be developed.

Concerning the size of the blobs, it is also worth noting that increasing $\sigma$, thus the size of the analysis window in the WFT, leads the blobs to be spread out, which is again observed in practice (not illustrated here). Finally, high strain gradients would certainly benefit of taking into account the limited resolving power inherent to any optical device, and possibly to characterize the point spread function (PSF) of the lens.

\subsection{Lens artefacts}

As any white-light technique such as DIC ([162, 161]) relying on cameras equipped with lenses, the grid method is prone to lens artifacts such as vignetting and geometric distortion.

Vignetting (or peripheral shading) corresponds to a light fall-off towards the corners of the grid images. Its effect can be easily understood with Equation 6.1, since light fall-off causes the modulus $K$ of the output of the WFT to decrease, and thus the noise due to camera sensor to increase towards the edges of the phase and phase derivative maps, so the displacement and strain maps since the displacement of the physical point between current and reference configurations is generally very small in amplitude.

Lenses are also known to be impaired by geometric distortions. Straight lines drawn on the specimen may not be mapped to straight lines on the sensor, giving for instance the so-called barrel and pincushion radial distortions, or even more complex distortions [181]. Of course, distortions affect the estimate of displacement maps based on the phase modulations of grid images. In Ref. [182] this effect is even proposed to be considered as a cheap and convenient way to assess this distortion. We focus on the influence of the most prominent distortions, namely radial distortions, on the displacement and strain maps since this effect can be easily studied.

Geometric distortions can be modelled by additional phase changes $\Theta_{x}$ and $\Theta_{y}$ which have the same distribution in the images taken before and after deformation. Identifying the phase modulations as in 4.8 gives:

$$
\left\{\begin{aligned}
\phi_{x}^{2}\left(x+u_{x}(x, y), y+u_{y}(x, y)\right) & +\Theta_{x}\left(x+u_{x}(x, y), y+u_{y}(x, y)\right)=-2 \pi f u_{x}(x, y) \\
& +\phi_{x}^{1}(x, y)+\Theta_{x}(x, y) \\
\phi_{y}^{2}\left(x+u_{y}(x, y), y+u_{y}(x, y)\right) & +\Theta_{y}\left(x+u_{y}(x, y), y+u_{y}(x, y)\right)=-2 \pi f u_{y}(x, y) \\
& +\phi_{y}^{1}(x, y)+\Theta_{y}(x, y)
\end{aligned}\right.
$$

Geometric distortions thus change the fundamental relation given by 4.12 to

$$
\Delta \phi(\boldsymbol{x})=-2 \pi f \boldsymbol{u}(\boldsymbol{x})+\boldsymbol{\Theta}(\boldsymbol{x})-\boldsymbol{\Theta}(\boldsymbol{x}+\boldsymbol{u}(\boldsymbol{x}))
$$


We can see that geometric distortions give an additional bias in the estimation of the displacement field. Several parametric models for geometric distortions are available in the literature, see for instance [183] or [184] where algorithms for parameter estimation are also proposed. In the simplest model, geometric distortions make a pixel $\boldsymbol{y}$ in the undistorted image to be mapped to

$$
\boldsymbol{x}=\boldsymbol{y}+k\left(\boldsymbol{y}-\boldsymbol{y}_{0}\right)\left\|\boldsymbol{y}-\boldsymbol{y}_{0}\right\|^{2}
$$

where $\boldsymbol{y}_{0}$ is the principal point, that is, the intersection of the optical axis with the image plane, $\|\cdot\|$ is the Euclidean norm, and $k$ is the distortion coefficient (either positive for pincushion distortion or negative for barrel distortion). The relative distortion $k\left\|\boldsymbol{y}-\boldsymbol{y}_{0}\right\|^{2}$ is typically below $1 \%$, a value of $5 \%$ representing a massive distortion.

Distortion is thus equivalent to a virtual direct displacement $\overline{\boldsymbol{u}}(\boldsymbol{y})=k\left(\boldsymbol{y}-\boldsymbol{y}_{0}\right)\left\|\boldsymbol{y}-\boldsymbol{y}_{0}\right\|^{2}$. Note that $\overline{\boldsymbol{u}}\left(\boldsymbol{y}_{0}\right)=0$. Specifying (4.6) to $\phi_{2}=\boldsymbol{\Theta}$ and $\phi_{1}=0$, we obtain $\boldsymbol{\Theta}(\boldsymbol{x})=2 \pi f \overline{\boldsymbol{U}}(\boldsymbol{x})$, where $\overline{\boldsymbol{U}}$ is the inverse displacement associated with $\overline{\boldsymbol{u}}$. The bias in $(6.6)$ thus writes as follows:

$$
\boldsymbol{\Theta}(\boldsymbol{x})-\boldsymbol{\Theta}(\boldsymbol{x}+\boldsymbol{u}(\boldsymbol{x}))=2 \pi f(\overline{\boldsymbol{U}}(\boldsymbol{x})-\overline{\boldsymbol{U}}(\boldsymbol{x}+\boldsymbol{u}(\boldsymbol{x})))
$$

It is interesting to note that, when the displacement field $\boldsymbol{u}(x)$ is negligible, the phase difference simply discards the effect of the distortion, even for quite strong distortions which could affect the corners of the imaged grids. In general, displacement gives however an additional bias, which is all the larger as the point under consideration is far away from the principal point. The question is: to what extent does the bias impair the displacement field?

The derivatives of the smooth function $\overline{\boldsymbol{u}}$ being small with respect to $\overline{\boldsymbol{u}}$, the approximation $\overline{\boldsymbol{U}}=-\overline{\boldsymbol{u}}$ is valid. Consequently,

$$
\boldsymbol{\Theta}(\boldsymbol{x})-\boldsymbol{\Theta}(\boldsymbol{x}+\boldsymbol{u}(\boldsymbol{x}))=-2 \pi f k\left(\left(\boldsymbol{x}-\boldsymbol{y}_{0}\right)\left\|\boldsymbol{x}-\boldsymbol{y}_{0}\right\|^{2}-\left(\boldsymbol{x}+\boldsymbol{u}(\boldsymbol{x})-\boldsymbol{y}_{0}\right)\left\|\boldsymbol{x}+\boldsymbol{u}(\boldsymbol{x})-\boldsymbol{y}_{0}\right\|^{2}\right)
$$

Now, equalling both squared norms in Equation 6.9 (small displacement assumption) gives the following first-order approximation in $\boldsymbol{u}$ :

$$
\boldsymbol{\Theta}(\boldsymbol{x})-\boldsymbol{\Theta}(\boldsymbol{x}+\boldsymbol{u}(\boldsymbol{x})) \simeq 2 \pi f k \boldsymbol{u}(\boldsymbol{x})\left\|\boldsymbol{x}-\boldsymbol{y}_{0}\right\|^{2}
$$

With the typical values for the relative distortion $k\left\|\boldsymbol{x}-\boldsymbol{y}_{0}\right\|^{2}$ (typically around $1 \%$ ), this means that the bias in the displacement measurement can be confidently neglected in (6.6). Moreover, since the imaged grid and the specimen are very small, fix-focal macrolenses are used in practice. Most of the time, these lenses are well designed with respect to geometric distortions. For instance, the Sigma 105mm macro-lens has a maximal relative distortion measured below $0.1 \%$ with a $36 \times 24 \mathrm{~mm}$ sensor. Since CCD sensors that are used in our experimental setting are smaller, the actual relative distortion is even smaller than $0.1 \%$.

Concerning the phase difference derivatives (which provide the strain components), 6.10) gives for example:

$$
\frac{\partial}{\partial x}(\boldsymbol{\Theta}(\boldsymbol{x})-\boldsymbol{\Theta}(\boldsymbol{x}+\boldsymbol{u}(\boldsymbol{x})))=2 \pi f k \frac{\partial \boldsymbol{u}}{\partial x}(\boldsymbol{x})\left\|\boldsymbol{x}-\boldsymbol{y}_{0}\right\|^{2}+4 \pi f k\left(x-y_{0}\right) \boldsymbol{u}(\boldsymbol{x})
$$

$\left(x-y_{0}\right)$ being the first component of $\boldsymbol{x}-\boldsymbol{y}_{0}$. The bias induced by geometric distortion on the strain component estimation has two components. The first one is negligible, as in the displacement measurement. The second one, however, is proportional to the displacement and is all the stronger as $\boldsymbol{x}$ is away from the optical center. It means that, if 
the strain is very small but the displacement is quite large (which is the case in a rigid body motion), then optical distortion is likely to give dubious strain measurements, especially in the image corners. This was noted in [161] concerning DIC. However, the term $k\left(x-y_{0}\right)$ is at most around $10^{-5}$ at a distance of 1000 pixels of the optical center of the above lens, since the relative distortion $k\left\|\boldsymbol{x}-\boldsymbol{y}_{0}\right\|^{2}$ is itself below $1 \%$.

It should be noted that, if the geometric distortions cannot be neglected (for example in the above-mentioned situation, or in a low cost system with severe distortions), most image processing software propose an automatic numerical correction which could be advantageously used, as in [161], but the impact on the metrological performance of the underlying subpixel interpolation should be assessed in this case.

\subsection{Verification of the theoretical prediction for the strain and displace- ment resolutions}

The theoretical predictions given in Equations 5.10 were experimentally verified in [78]. Two specific preprocessing procedures had however to be applied to correctly estimate the noise in grid images:

1. Camera sensor noise heteroscedasticity. Noise variance stabilization with the Generalized Anscombe Transform. Equations 5.10 are obtained assuming that the sensor noise $\sigma_{\text {image }}$ is Gaussian and has a standard deviation which is constant throughout the image, or at most only gently spatially changes over the surface covered by the analysis window of the WFT. This assumption is commonly accepted in the Photomechanics community (see for instance [155] concerning the determination of the noise in displacement maps obtained by DIC), mainly because calculations are not tractable if $\sigma_{\text {image }}$ spatially changes. This is however a rough assumption since actual camera sensor noise is Poisson-Gaussian and heteroscedastic (or signaldependent): the higher the brightness, the higher the noise level [185], and noise variance in images may reasonably be modeled with an affine function of the grey level [186, 187, 188]. Since we have several grid periods over the surface covered by the analysis window, $\sigma_{\text {image }}$ significantly changes over this surface, thus making this assumption of homoscedastic noise not to be rigorously satisfied. It is shown in [78, that feeding Equations 5.10 with the equivalent standard deviation of the noise (defined as the square root of the sum of the variances at any pixel of the images), leads Equations 5.10 to be satisfied from a global point of view only. In Ref [78], it is proposed to solve this problem by applying the so-called Generalized Anscombe Tranform, which changes a heteroscedastic Poisson-Gaussian noise into a homoscedastic Gaussian one [189, 190. Applying this transform stabilizes the standard deviation of the noise, and finally makes Equations 5.10 to be satisfied at the local level. This is a non-linear transform, so a bias is induced by applying it. It is however assumed to be negligible compared to the other sources of errors. A similar approach has been applied recently for DIC [100, 101].

2. Influence of micro-movements between camera and specimen on the characterisation of the noise in grid images Note that assessing the noise level at any pixel by analyzing a stack of images may be corrupted by micro-movements between camera and sensor. A specific image processing proposed in Ref. [191] enables us to get rid of the influence of this movement on the camera sensor noise estimation from a stack of grid images. Interestingly, it is shown in Ref. [192] that time averaging a stack of images affected by a micro-movement between grid and camera provides a biased 
estimator of the noiseless grid image, but that phases and phase derivatives (thus displacements and strains) which are deduced from these "corrupted" mean grid images are not affected for magnitudes of movements generally observed in practice (some $\mu \mathrm{m}$ in amplitude).

\subsection{Grid defects}

The grid method relies on the use of a spatial carrier and the sought information is contained in the modulation of this carrier. The modulation may be due to deformation, but also to printing issues. Grids must indeed be printed, but any printing technique has certain limits in terms of regularity of the line network. Fluctuations of the grid pitch throughout grid images may lead to phase and phase derivative fluctuations which can be interpreted as caused by fictitious strains. Local lacks of ink may also occur, inducing some "blobs" to appear in the phase and mainly phase derivative maps. Such phenomena can be noticed even with high-resolution printing devices. They clearly corrupt the resulting phase derivative maps if Procedure 2 described in Section 4.2 .2 is not applied. This point is illustrated below through a representative example: the measurement of the displacement and strain fields around the hole of a open-hole specimen subjected to a tensile test. Figure 11 a shows the displacement map obtained for an applied loading such that plasticity occurs between the hole and the specimen border. It is obtained by using the Procedure 1 in Section 4.2 .2 (one-step approach). The displacement field is rather smooth although the phase is not subtracted at the same physical points, and only slight localized horizontal "waves" are detectable to the naked eye (see for instance the arrows denoted A). These waves are indeed due to grid pitch fluctuations.

Differentiating this displacement field gives the $\varepsilon$ strain field shown in Figure 11 b. Two types of defects are visible to the naked eye here:

- thin horizontal lines: they are caused by slight grid pitch defect due to printing, as shown in [99] and [80] (for 1D and 2D grids, respectively)

- localized "blobs" denoted B and C in Figure 11-a). This is the consequence of local marking defects such as small bubbles or dust which are not really avoidable in practice when marking the specimen.

These defects are also visible in the phase derivative map, as illustrated in Figure 11 c, which represents the $\frac{\partial \Phi_{y}}{\partial y}$ phase derivative map in the reference (on the left-hand side) and the current (on the right-hand side) configurations. Some defects are the same in distribution and shape as in Figure 11 -b, see for instance blob B. Blob C is not visible. This is logical since the latter comes from the reference phase derivative map. The pairs of blobs in Figure 11 $\mathrm{b}$ are due the fact that the subtraction between current and reference phase maps is not performed exactly at the same physical point with the first procedure: defects are thus not eliminated, but appear twice on the maps and with an opposite sign due to the subtraction, as clearly visible when comparing Figure 11 -b and 11 -c. The effect of the vertical displacement can be seen along the border between the left- and right-hand side sub-figures in Figure 11-c: the amplitude of the discontinuity along the $y$-direction is directly equal to the amplitude of the vertical displacement.

The strain field in Figure 11-d is obtained by using the procedure proposed in [80. It is equivalent to Procedure 2 in Equations 4.18 and 4.19, in which the displacement field has converged after one iteration, the strain components are negligible compared to unity and the effect of the rotation is also negligible. It can be seen that the defects have almost 


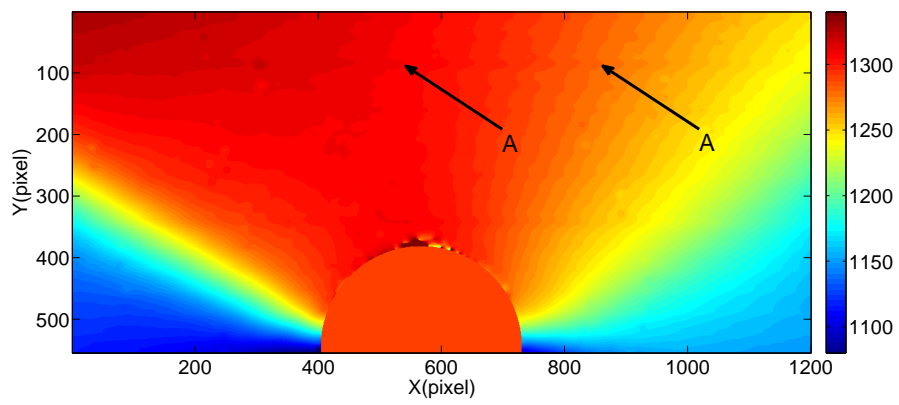

a-

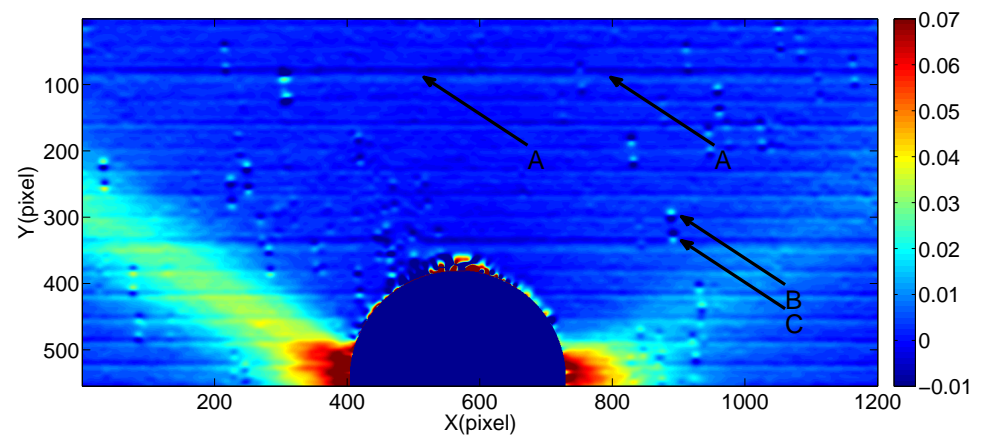

b-

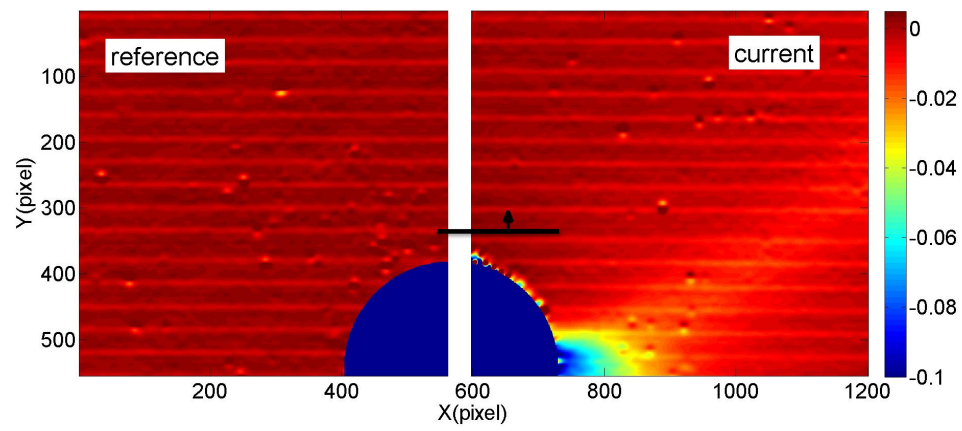

C-

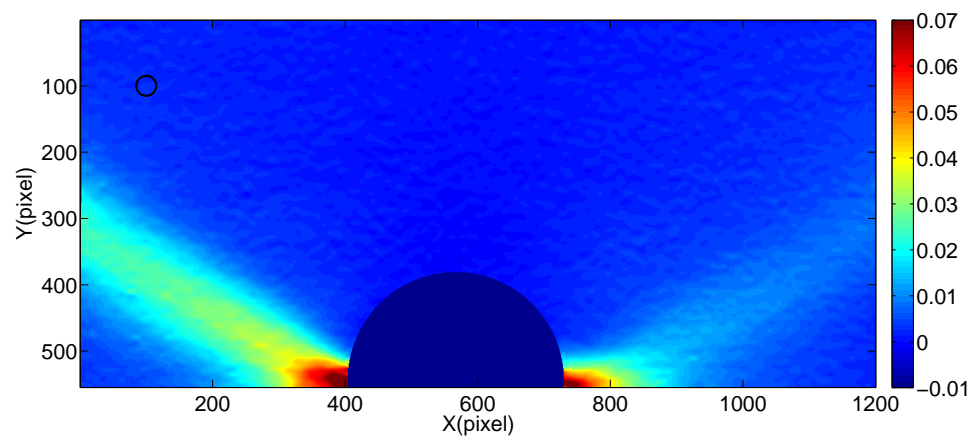

d-

Figure 11: Illustrative example of the impact of grid defects on the displacement and strain maps, and defect removal by displacement compensation. a- vertical displacement $u_{y}$ (in micrometer). b- $\frac{\partial u_{y}}{\partial y}$ distribution. c- $\frac{\partial \Phi_{y}}{\partial y}$ distribution. Left: in the reference configuration. Right: in the current configuration. A slight vertical movement is visible between the two. d- $\varepsilon_{y y}$ with displacement compensation. dimensions in pixels, 1 pixel represents $40 \mu \mathrm{m}$ on the specimen 
completely disappeared. In addition, the borders of the features in the geometry of the specimens are better resolved. The main benefit is to avoid any classic spatial filtering of the localized defects visible in Figure 11 b, which is interesting because filtering potentially impairs the spatial resolution of the measuring technique, and causes actual details in strain maps to be blurred, as illustrated by the relative bias which increases. The diameter of the black circle superimposed to the map at the upper left corner of Figure11.d is equal to $6 \sigma$, in other words to the spatial resolution $d_{1 \varepsilon}$ of the strain measuring technique. We have the same value for the spatial resolution as in the preceding maps, thus illustrating the fact that no loss of spatial resolution is induced when going from Figure 11 b to Figure 11 d, even though the defects are eliminated.

\subsection{Parasitic fringes in strain maps}

In certain cases, parasitic low-frequency fringes may appear in the grid images themselves. Even if they are generally barely visible to the naked eye, they may then induce lowfrequency parasitic fringes in the strain maps when the actual strain amplitude is small. There are various causes, but the main one seems to be aliasing. Indeed the regular pattern of the grid is sampled by camera sensor, and this may induce aliasing if the Nyquist condition is not satisfied in Petersen-Middleton sampling theorem [193] (which is a generalization to images of the 1D Nyquist-Shannon sampling theorem). This phenomenon does not always appear, depending for instance on the type of camera and on the focus. Defocusing, which simulates a low-pass filter enforcing the Nyquist condition, leads these fringes to disappear or to be drastically limited, but this also reduces the contrast in the grid images, and thus causes the noise to be higher in the strain maps according to Equation 6.1.

Figure $[12$ a illustrates these spurious fringes. We have here a Portevin-Le Chatelier band which appears in some aluminium specimens subjected to tensile tests. This map is the strain increment distribution observed between two consecutive images shot during a tensile test. In this case, the parasitic fringes are not visible on the strain maps themselves (because of the actual strain amplitude is too high), but on the strain increment maps. In Ref. 194 it is shown that such parasitic fringes may be removed by filtering in the frequency domain. The procedure consists first in detecting the peaks corresponding to these parasitic fringes in the Fourier domain, and then in designing a suitable notch filter which is used to erase these peaks. Taking the inverse Fourier transform leads to the filtered map. Figure 12 -b shows the filtered distribution of strain increments, and Figure 12 -c the difference between raw and filtered strain increment distributions. Full details on this procedure are given in [194]. Designing automatically such a notch filter for strain maps remains an issue while this is not the case for natural images. The reason is that designing automatically such a filter relies on an assumption concerning the decay of the coefficient representing the image in the power spectrum: this decay follows a power law model for natural images but this is generally not the case for strain maps [195]. Finding a model suitable for strain maps still remains an open= question.

Very recently, it has been proposed in [176] to rotate the grid with respect to the lines and columns of pixels of the camera sensor. It is shown that this trick strongly limits this undesirable phenomenon. This procedure is of course recommended to avoid filtering parasitic fringes in the Fourier domain 

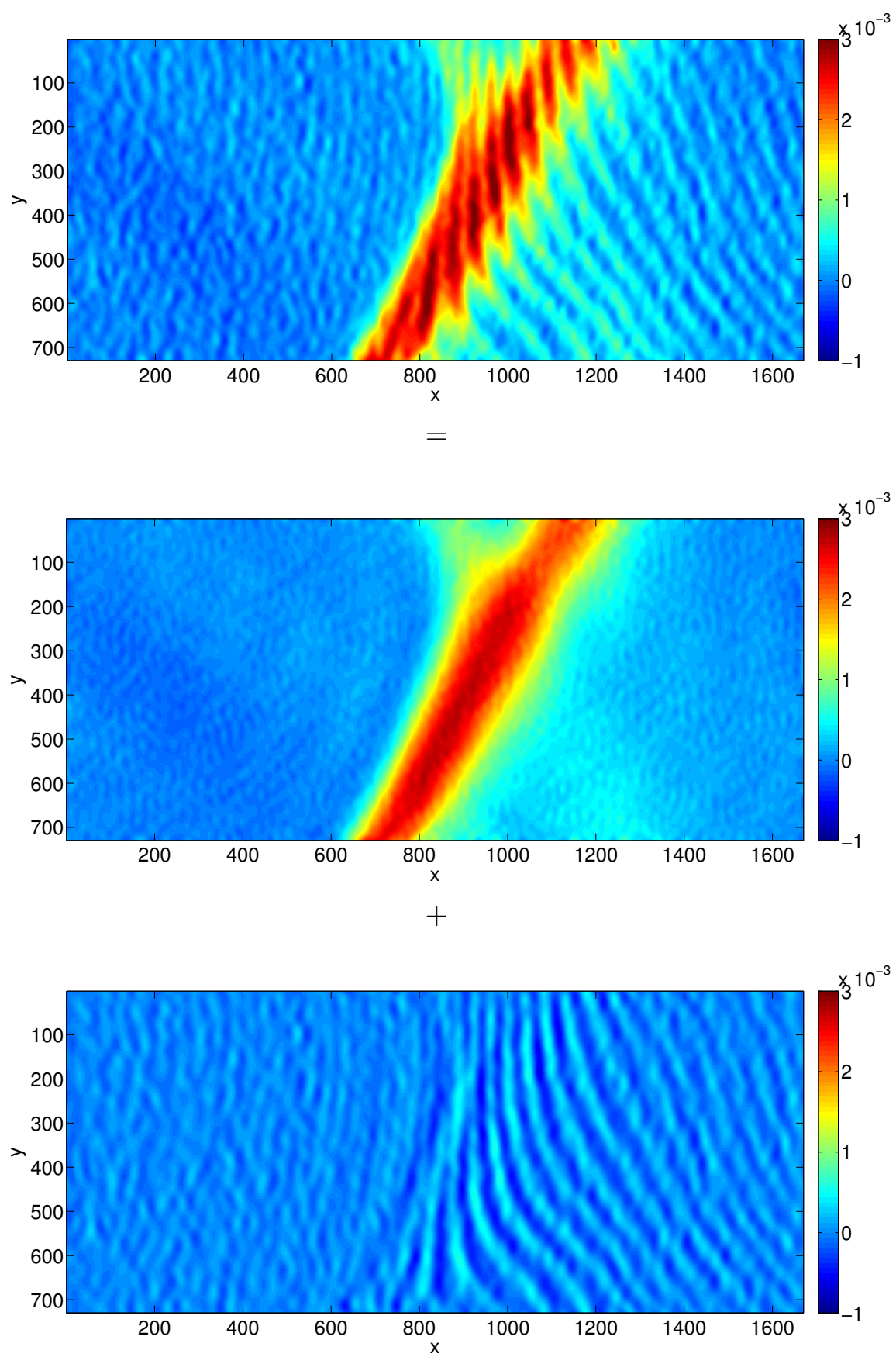

Figure 12: Removing parasitic fringes by filtering in the frequency domain, after [194] 


\section{Examples}

\subsection{Introduction}

The idea here is to give some examples of use of the grid method in experimental mechanics, in situations for which it is relevant to measure displacement and strain fields to characterize the mechanical properties of materials and structures. Even though grids are reported to be used as local displacement transducers, see for instance [196] in the case of large structures such as pipes of power plants or [75, 76] for ceiling traveling cranes, fields of measurements are generally obtained and analyzed with this technique.

Two main cases must be distinguished, depending on the use of these full-field measurements:

1. full-field measurements can be employed to detect phenomena through strain or displacement heterogeneities in the corresponding maps, to see if "something happens somewhere in the specimen". Qualitative measurements are sufficient in this case. However, there is often a great temptation to go further by getting quantitative information concerning the observed phenomenon, by measuring the displacement or strain amplitude at some points or over some zones. This task is more demanding than a simple observation since the metrological performance of the system should be provided along with the output of the measuring system. This is however rarely the case;

2. in the second case, the idea is to go further by identifying the parameters governing any relevant model that can describe the observed phenomenon. The most current case consists in retrieving the parameters governing the constitutive equations of the tested material.

We propose hereafter to illustrate these two cases through a series of examples published in the literature concerning in-plane displacement. The reader interested in plate bending and/or slope measurement problems is referred to 197, 198, where a technique similar to the grid method, namely deflectometry, is described and employed in various cases of material characterisation with plate in bending [199, 200, 201, 202].

\subsection{Observing and quantifying heterogeneities in displacement and strain maps}

\subsubsection{Tracking crack propagation}

The easiest case (in terms of ability to detect a phenomenon causing strain gradients to appear in a strain map) is when a "significant" heterogeneity occurs, the ultimate situation being a discontinuity. A crack in a displacement or strain field matches this definition. This has led the grid method to be employed in this case. In [69, 204], it was used to detect the presence of cracks in concrete beams reinforced with composite plates bonded on their soffit. The crack width was measured along the high of the specimen, while the strain fields enabled the authors to decompose crack opening in the shear span in mode I and mode II. A similar study was carried out in [70] where the cracking process of beams made of a cement-based fibre composite was examined. Beams were tested in three- and four-point bending and the cracking pattern was deduced in each case, enabling to analyze the cracking evolution during the test, and establish the link between the crack pattern and the global response of the tested specimens. An aluminium notched specimen was tested in [203]. Interestingly, the specimen was tested in an Arcan device 

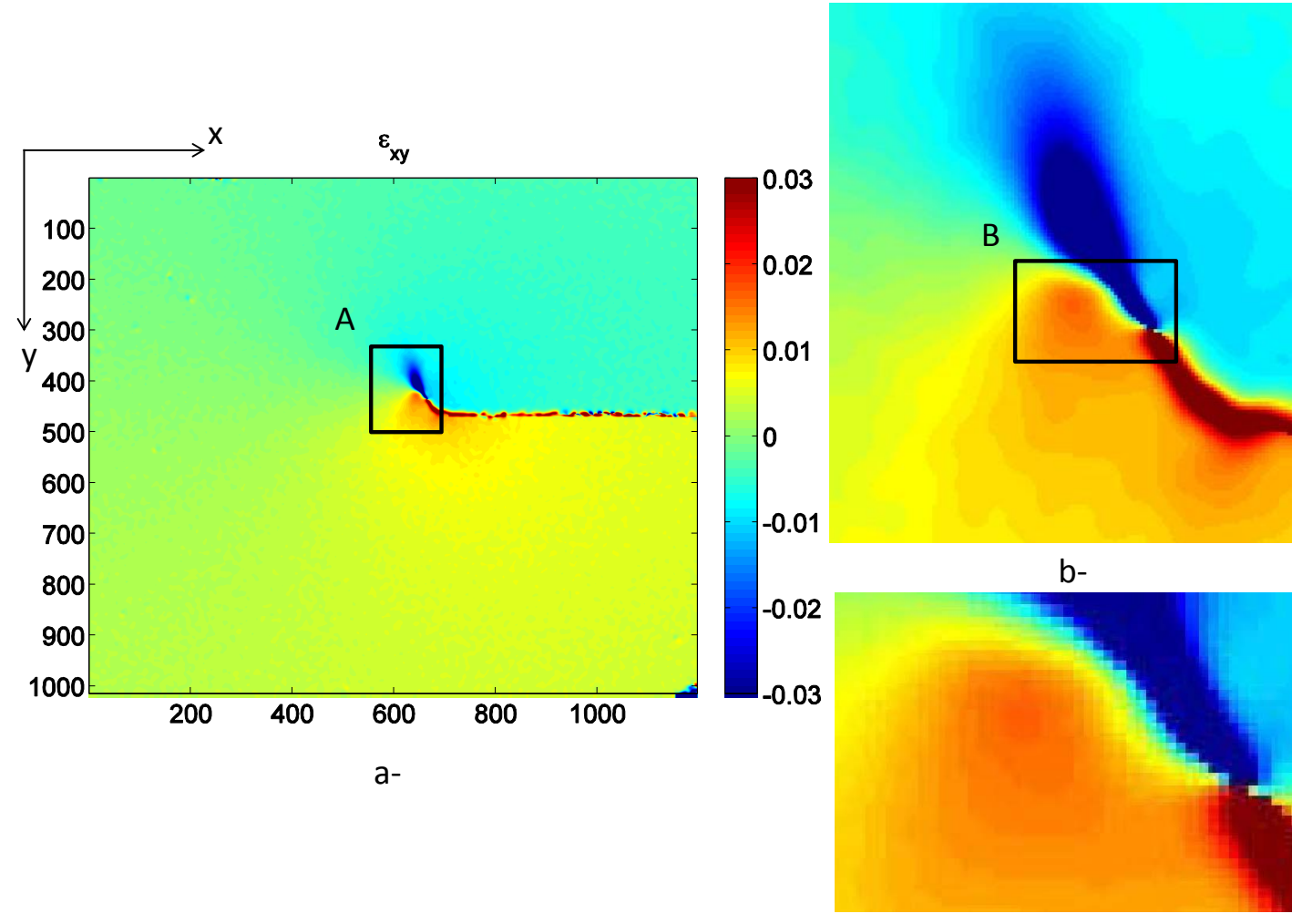

C-

Figure 13: Strain field near a crack tip in aluminium specimen (see [203]). dimensions in pixels, 1 pixel represents $40 \mu \mathrm{m}$ on the specimen. a- shear strain field. b- close-up of zone A. c- close-up of zone B

beforehand, with a load direction inclined with respect to the normal direction of the notch, causing a crack to initiate along a direction also inclined with respect to the notch. The strain and local rotation fields were measured, especially near the crack tip. The crack tip location was found with a sub-pixel resolution, by finding the crossing point of different valleys in the strain distribution. A typical example of shear strain map at the crack tip is first depicted in Figure 13. The crack, which is clearly distinguishable, is inclined with respect to the horizontal direction. The dimension covered by Figure 13 . a is about 1000 pixels in height and slightly more in width. The height is thus about $4 \mathrm{~cm}$ since one pixel correspond to a square which is $40 \mu \mathrm{m}$ in size. Two successive close-ups show the high strain gradients that occur near the crack tip, which is located between the red and blue spots. In particular, the pixelwise distribution is clearly visible in Figure 13-c, illustrating the fact that no interpolation is performed when calculating the stain distribution and representing the obtained result. It is worth remembering that the measured strain gradient, albeit significant, is lower than the actual one since the measured strain distribution is equal, at first approximation, to the actual one convolved by the analysis window of the WFT (here a Gaussian window), as explained in Section 5. The actual strain distribution would be obtained by deconvolving the measured strain distribution.

The response of wood has also been observed and analyzed with the grid method: the influence of the orientation of annual rings on the crack propagation in notched beams was discussed for instance in [205]. The most interesting point here is to observe the 


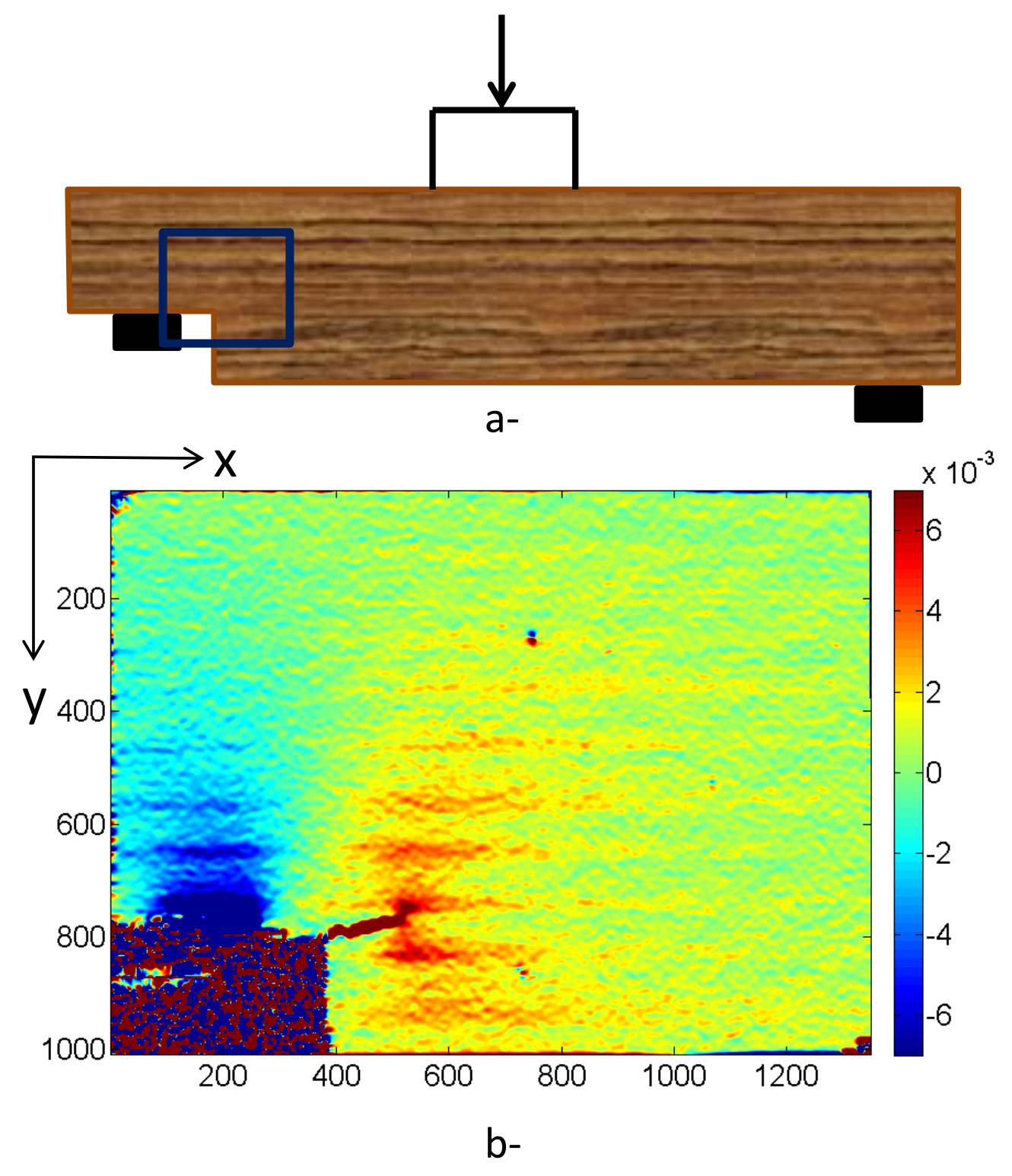

Figure 14: Transverse strain field $\varepsilon_{y y}$ near the notch of a cracked notched wood beam specimen (see 205]). a- Schematic view of the notched beam. b- Strain field near the notch. dimensions in pixels, 1 pixel represents $20 \mu \mathrm{m}$ on the specimen 
heterogeneity in the strain field, which is closely related to the very heterogeneous nature of the material itself (because of the annual rings). Figure $14 \mathrm{ta}$ is a schematic view of the tested beam. It has a notch on the left-hand side, which induces transverse strain to appear in the current section of the beam, next to the notch, and a crack to initiate at the right angle because of this transverse normal strain. Figure 14 b shows that it is possible to analyse this effect with the grid method. In particular, the transverse tensile strain is clearly visible at the right-hand side of the notch (red spot) while a compressive zone also appears above the support (blue spot). The most stricking fact is the ability to distinguish the annual rings of the wood, revealed here by the horizontal "waves" in the strain maps. They are caused by the contrast in stiffness between early and late woods. Finally, it is worth mentioning that the strain map shown here is corrupted by "blobs" which did not appear in the preceding example. This is due to the fact that the actual strain value is lower here than in the preceding cases. It was also not possible to perform time-averaging to limit the sensor noise influence (the standard deviation of this noise is theoretically divided by $\sqrt{N}$, where $N$ is the number of frames averaged to provide one grid image), because crack propagation is a dynamic effect, the crack propagating suddenly and stepwise as the imposed displacement increases regularly. This example briefly illustrates the current limit of this strain measuring technique, since analyzing phenomena with lower strain amplitudes would obviously lead the noise to be more visible with the same settings, so without impairing the spatial resolution.

\subsubsection{Local strain heterogeneities due to the heterogeneous nature of the material}

Significant local strain heterogeneities may be due to the fact that the material itself features heterogeneous mechanical properties, caused for instance to the presence of different phases. This is the case in various examples for which the grid method was employed. In [207] for instance, the heterogeneous plastic response of an aluminum multicrystal was revealed through strain maps. This heterogeneity is caused by the difference in orientation from one grain to another, which also leads to a difference in the slipping system of the atomic lattice. Shape memory alloys feature peculiar properties which are due, among others, to a phase change under stress. This is what is observed in [206], where the appearance and growth of martensite needle in an austenitic material is revealed during a tensile test on a $\mathrm{Cu}-\mathrm{Al}-\mathrm{Be}$ monocrystal. The strain intermittency cause by the austenite $\rightarrow$ martensite phase transform in the same material was characterized in [208. Figures 15-a and -b show two typical $\varepsilon_{x x}$ strain maps measured during this study (the magnitude of the load is higher in Figure 15-b). Narrow inclined bands visible on a blue background reveal the austenite $\rightarrow$ martensite phase transformation that occurs while loading the specimen. Inclined martensite needles going through the specimen are also visible. The length of the gauge section of the specimen is equal here to $17.78 \mathrm{~mm}$ and the height of the map to $53.00 \mathrm{~mm}$. This gives an idea of the size of the features visible in these maps. Figure 15 c represents the typical shape of the specimen under load: warping (because the tensile direction is not aligned with one of the symmetry axes of the crystal) occurs in addition to a vertical stretch. This realistic view of the deformed specimen is obtained by adding the (magnified) measured displacements to the coordinates of the nodes of a mesh of the specimen. A warping occurs and localized inclined bands appear. They reveal the localized aspect of the phase transformation.

In civil engineering, asphalt is by essence a strongly heterogeneous material, since stiff aggregates are mixed with a soft bitumen matrix. Compressive tests carried out on such specimens permitted to analyze this heterogeneous nature thanks to the grid method 


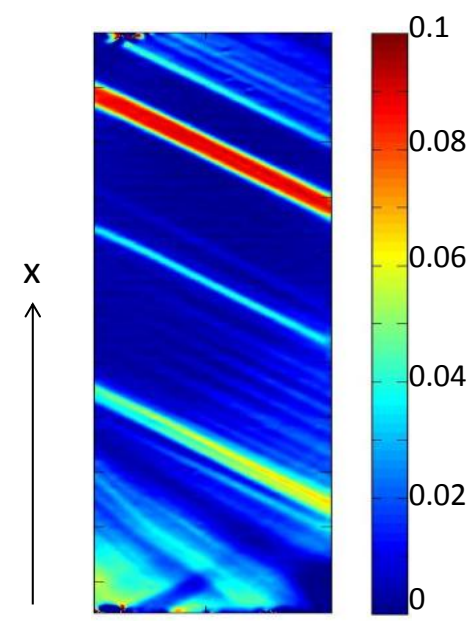

a-

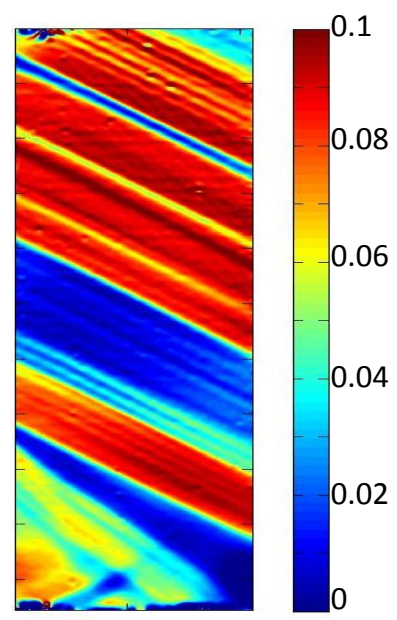

b-

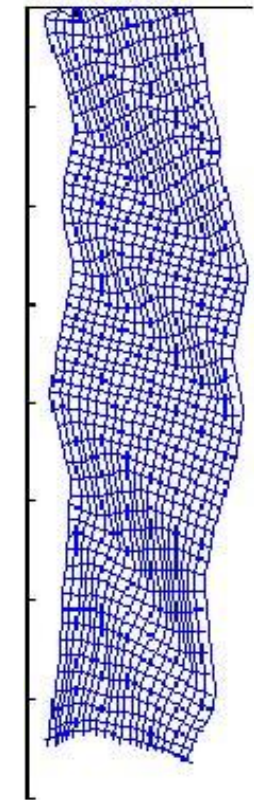

C-

Figure 15: Tensile test on a monocrystal of shape memory alloy (see [206]). a- and btypical $\varepsilon_{x x}$ strain fields for different values of the load. c- typical deformed specimen during the test. dimensions in pixels, 1 pixel represents $40 \mu \mathrm{m}$ on the specimen

in 209. The same technique was used in 210] to compare two different asphalt mixes, and in 211] to study the mechanical response of reclaimed asphalt pavements. Figure 16 -a shows the front face of an asphalt specimen before marking the surface with a grid (full details in [209]) as well as a typical $\varepsilon_{y y}$ strain maps measured during a compression test along the vertical direction. The same pattern as that visible on the front face is distinguishable on this strain map in Figure 16 b. For instance, aggregates A and B are clearly recognizable in the strain map. This is due to the fact that the big difference in stiffness between aggregates and bitumen binder causes high strain gradients to appear. The principal directions are superimposed to this distribution, with portions of lines proportional to the eigenvalues for the strain (color code: compression in blue, extension in red). As shown in Figure 16 c, the deformed specimen is barrel-shaped. Its top and bottom boundaries are not exactly subjected to a uniform vertical displacement while loading, illustrating that real boundary condition may significantly differ from the desired one (this is due here to the flexibility of the steel plates interposed between specimen and grips of the testing machine). The displacement of the aggregates is also superimposed as well as there rotation, which enables then a thorough analysis of the link between global response of the specimen and local response of the constituents during the test, as discussed in [209].

As already mentioned above, the heterogeneous nature of wood may potentially appear in heterogeneous strain fields in wood specimens when they are mechanically loaded. In 212, the heterogeneous hygroscopic response of the hydric transfer phenomenon was 


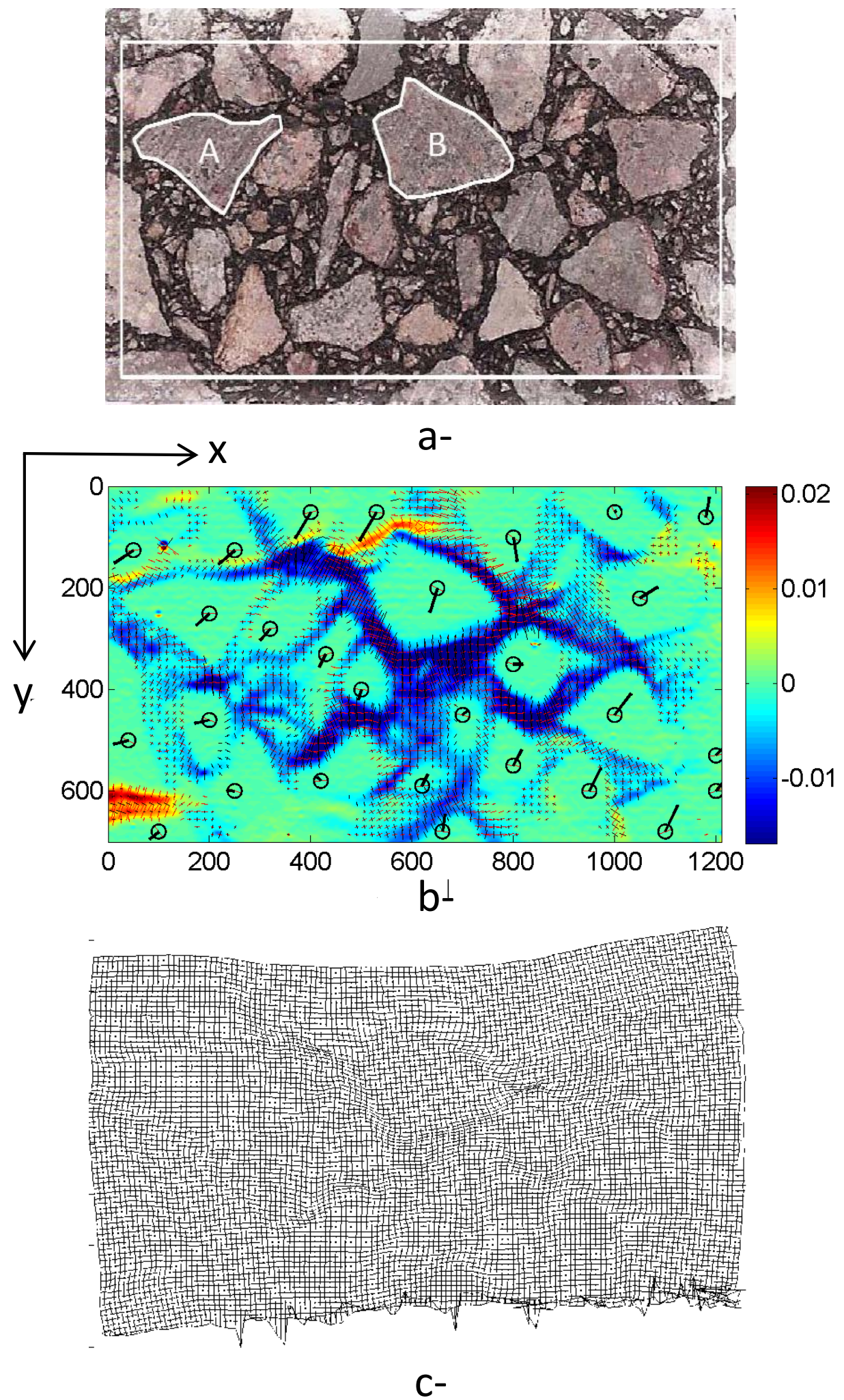

Figure 16: Asphalt specimen under vertical compression. a- picture of the front face before applying the grid. b- $\varepsilon_{y y}$ strain fields for a given value of the load (see [209]). The correspondence between the two sub-figures is clearly visible. c- deformed specimen. Dimensions in pixels, 1 pixel represents $40 \mu \mathrm{m}$ on the specimen 
studied in the same spirit. Displacement and strain fields were analyzed in batteries in 213 using the sampling moiré technique. Interestingly, the periodic nature of the material itself was used as natural marking in this case.

Finally, the use of the grid method at the microscopic scale is reported in various papers, see for instance Refs. [214, 215, 216] for measuring strain near a dislocation, [217] for observing singularities like disclinations in nanoparticles, [218, 77] for measuring residual stresses, [219, 220] for measuring deformation near a crack tip. Strain fields were also measured in many other situations, for instance around low-angle grain boundaries [221], in silicon transistors [222], in nanowires [223] or in MEMS devices [224]. The geometric phase analysis (GPA) briefly described in Section 4.4.1 was employed in these last examples.

\subsubsection{Local strain heterogeneities due to the geometry of the specimen}

Strain fields may also be heterogeneous because of geometrical singularities. Apart from the ultimate case of cracking already discussed above, strain distributions may spatially change because of a change in geometry of the tested specimen. In [225] for instance, heterogeneous strain fields appearing in a cover-plate bolted steel joints were studied. In this study, the heterogeneous nature of the strain field was caused by the holes drilled in the plates and the loading applied by the bolts.

\subsection{Identification from full-field measurements}

The idea here is to go beyond strain measurements to identify parameters governing constitutive equations or the phenomena observed with full-field measurements. Heterogeneous strain fields involve a greater number of constitutive parameters than homogeneous strain fields. If a suitable identification procedure is employed, these parameters can be retrieved. Compared to the classic situation for which homogeneous strain fields are employed to identify parameters (as in the case of the usual tensile test on parallelepipedic specimens) the benefit is to retrieve a greater number of parameters at the same time, thus potentially reducing the number of tests to be performed. Processing heterogeneous strain is even unavoidable in the case of heterogeneous materials.

Identification methods suited to the case of full-field measurements have been presented in a review paper [226]. However, whatever the method used, retrieving reliable values of constitutive parameters means that the strain fields considered as input for the identification procedure are reliable too. As mentioned above, some mechanical characteristics such as the bias are signal-dependent. When considering average values for the sought parameters, this is however not really an issue: strain values are potentially underestimated in some zones (sharp peaks in the strain distribution), but this can be globally counterbalanced by the strain values which are overestimated in other zones (narrow valleys in the strain distribution).

The grid method has been successfully employed in various cases of constitutive parameters in the literature. Within the framework of linear elasticity, let us mention the case of wood [227, 228] and composite [229, 230]. Damage can be considered as a mere extension in some cases [231, 232]. Plasticity involves higher strain levels, see [233, 234, 235]. Even though specific problems are raised by dynamics measurements [236], the use of the grid method is reported is recent papers [237, 238, 239, 240] in this particular case. 


\section{Conclusion}

The main features of the grid method were given in this paper. We mainly focused on its history, on the technique which seems the most efficient to process grid images, and on practical problems which may occur when using this measuring technique. Special emphasis was put on the metrological performance of the grid method, defined here by the measurement resolution, the measurement bias and the spatial resolution. The measurement resolution considered here is only that caused by sensor noise propagation, other sources such as grids defects being more difficult to assess. Various examples were finally shown in cases for which significant strain gradients occur in the tested specimen, a situation which is very challenging for full-field measuring systems.

At the moment, the dissemination of this technique is hindered by two main factors. First, the surface under investigation must be regularly marked by simple marking procedures, which are not really easily available. Second, grid image processing relies, in recent applications, on the windowed Fourier transform, a tool which is not really very common in the experimental mechanics community. This is however not the case in both the optics and the image processing communities. This leads to a wide literature available on this subject which could certainly be advantageously considered for measuring reliable displacement and strain fields in experimental mechanics. The main strengths of this technique are $i$ - from a practical point of view and bearing in mind that this is a white-light technique, the good compromise between measurement resolution and spatial resolution, ii- from a theoretical point of view, the fact that simple closed-form expressions are available between spatial resolution, measurement resolution and measurement bias when the WFT is performed for a frequency equal to the nominal pitch of the grid. Thus, depending on the goal the user wants to reach and the nature of the phenomenon he wants to characterize, the tradeoff between the three aforementioned metrological characteristics can be adjusted at best. All these features make the grid method a simple but efficient technique to measure 2D displacement and strain distributions which occur in deformed flat specimens, as illustrated in the examples shown at the end of this paper.

\section{Programmes}

Various programmes dedicated to grid image processing are available on the following website: www.thegridmethod.net, along with some information on the grid method. Readers interested in using by themselves this measurement technique are invited to download these programmes.

\section{Acknowledgements}

The French research network GDR CNRS ISIS is gratefully acknowledged for its partial financial support of this study (TIMEX project). The authors are also grateful to Drs Badulescu, Balandraud, Delpueyo, Fournely, Mouttou-Piti and Toussaint for their help in obtaining some of the figures shown in the paper. 


\section{References}

[1] P.K. Rastogi. Photomechanics. Topics in Applied Physic, volume 77. Springer, 2000. ISBN: 978-3-540-65990-7.

[2] M. Grédiac and F. Hild. Full-field measurements and identification in solid mechanics. Wiley, 2012. ISBN: 9781848212947, 496 pages.

[3] D. Tollenaar. Moiré interferentieverschijnselen bij rasterdruck. 1945. Institut voor Graphische Technik.

[4] P. Dantu. Utilisation de réseaux pour l'étude experimentale des phénomènes élastiques et plastiques. Comptes Rendus de l'Académie des Sciences, 239:17691771, 1954.

[5] R. Thiry and P. Dantu. Exposé général d'une méthode experimentale de détermination directe des déformations dans un solide. In 9ème congrès international de mécanique appliquée, Brussels, pages 490-497, 1957.

[6] P. S. Theocaris. Moiré fringes in strain analysis. Pergamon Press, Oxford, New York, 1969. 426 pages.

[7] P. Jacquot. Speckle interferometry: a review of the principal methods in use for experimental mechanics applications. Strain, 44(1):67-69, 2008.

[8] T. Kreis. Handbook of holographic interferometry: optical and digital methods. Wiley, 2004. ISBN: 978-3-527-40546-6, 504 pages.

[9] D. Post. Moiré interferometry: advances and applications. Experimental Mechanics, 31(3):276-280, 1991. Society for Experimental Mechanics.

[10] M. Sutton, J.J. Orteu, and H. Schreier. Image Correlation for Shape, Motion and Deformation Measurements. Basic Concepts, Theory and Applications. Springer, 2009. ISBN: 978-0-387-78746-6, 317 pages.

[11] B. Pan, K. Qian, H. Xie, and A. Asundi. Two-dimensional digital image correlation for in-plane displacement and strain measurement: a review. Measurement Science and Technology, 20:062001, 2009.

[12] S. Bossuyt. Optimized patterns for digital image correlation. In Proceedings of the 2012 Annual Conference on Experimental and Applied Mechanics. Volume 3: Imaging Methods for Novel Materials and Challenging Applications, 2013.

[13] V. J. Parks. The grid method. Experimental Mechanics, 9(7):27N-33N, 1969.

[14] P. S. Merill. Photogrid investigation of plastic-strain patterns in flat sheets with a hole. Experimental Mechanics, 1(8):73-80, 1961.

[15] R. W. Fail and C. E. Taylor. An application of pattern mapping to plane motion. Experimental Mechanics, 30(4):404-410, 1990.

[16] R. A. Ayres, E.G. Brewer, and S.W. Holland. Grid circle analyzer - computer aided measurement of deformation. SAE Technical Paper, 1979. Paper 790741.

[17] R. Sowerby, E. Chu, and J. L. Duncan. Determination of large strains in metal forming. The Journal of Strain Analysis for Engineering Design, 17(2):95-101, 1982. 
[18] E. Schedin and A. Melander. The evaluation of large strains from industrial sheet metal stampings with a square grid. Journal of Applied Metalworking, 4(2):143-156, 1986.

[19] J. H.Vogel and D. Lee. The automated measurement of strains from three dimensional deformed surfaces. The Journal of The Minerals, Metals and Materials Society, 42(2):8-13, 1990.

[20] D. W. Manthey and D. Lee. Vision based surface strain measurement system. The Journal of The Minerals, Metals and Materials Society, 47(7):46-49, 1990.

[21] T.A. Martin, G.R. Christie, and D. Bhattacharyya. Grid strain analysis and its application in composite sheet forming, volume 11. Composite Sheet Forming, chapter 6, pages 217-245. Elsevier, 1997. Composite Materials Series.

[22] H. Jin, S. Haldar, H.A. Bruck, and W.-Y. Lu. Grid method for microscale discontinuous deformation measurement. Experimental Mechanics, 51(4):565-574, 2011.

[23] X. Du, B. W. Anthony, and N. C. Kojimoto. Grid-based matching for full-field largearea deformation measurement. Optics and Lasers in Engineering, 66:307-319, 2015.

[24] W. Oliferuk, M. Maj, and K. Zembrzycki. Determination of the energy storage rate distribution in the area of strain localization using infrared and visible imaging. Experimental Mechanics, 55(4):53-760, 2015.

[25] T.C. Chu, W.F. Ranson, M.A. Sutton, and W.H. Peters. Applications of digital image correlation techniques to experimental mechanics. Experimental Mechanics, 25(3):232-244, 1983.

[26] L. Allais, M. Bornert, T. Bretheau, and D. Caldemaison. Characterization of the local strain field in a heterogeneous elastoplastic material. Acta Metallurgica et Materialia, 42(11):3865-3880, 1994.

[27] Experimental Mechanics. Special Issue: DIC Methods and Applications. 2015. 55(1). 22 articles, 311 pages, edited and prefaced by M. Sutton and F. Hild.

[28] J.L. Piro and M. Grédiac. Producing and transferring low-spatial-frequency grids for measuring displacement fields with moiré and grid methods. Experimental Techniques, 28(4):23-26, 2004.

[29] J. Notbohm, A. Rosakis, S. Kumagai, S. Xia, and G. Ravichandran. Threedimensional displacement and shape measurement with a diffraction-assisted grid method. Strain, 49(5):399-408, 2013.

[30] V.J. Parks. Strain measurement using grids. SPIE Optical Engineering, 21(4):633639, 1982.

[31] P. J. Sevenhuijsen. The photonical, pure grid method. Optics and Lasers in Engineering, 18(3):173-194, 1993.

[32] M. J. Hÿtch, E. Snoeck, and R. Kilaas. Quantitative measurement of displacement and strain fields from HREM micrographs. Ultramicroscopy, 74:131-146, 1998.

[33] M. J. Hÿtch and T. Plamann. Imaging conditions for reliable measurement of displacement and strain from high-resolution electron microscope images. Ultramicroscopy, 87:199-212, 2001. 
[34] F. Hüe, C.L. Johnson, S. Lartigue-Korinek, G. Wang, P.R. Buseck, and M.J. Hÿtch. Calibration of projector lens distortions. Journal of Electronic Microscopy, 54:181190, 2005.

[35] H. Xie, S. Kishimoto, A. Asundi, C. G. Boay, N. Shinya, J. Yu, and B. K. A. Ngoi. In-plane deformation measurement using the atomic force microscope moiré method. Nanotechnology, 1(11):24-29, 2000.

[36] H. Xie, G. B. Chai, A. K. Asundi, J. Yu, Y. Lu, B. K. A. Ngoi, Z. Zhong, and S. Kishimoto. AFM scanning moiré method for nanodeformation measurement. In G. M. Brown, W.. P. O. Jueptner, R. J. Pryputniewicz, M. Kujawinska, R. J. Pryputniewicz, and M. Takeda, editors, Proc. SPIE 4101, Laser Interferometry X: Techniques and Analysis, 2000.

[37] H. Xie, Z. Liu, D. Fang, F. Dai, H. Gao H, and Y. Zhao. A study on the digital nanomoiré method and its phase shifting technique. Measuring Science and Technology, 15:716-721, 2004.

[38] M. Tang, H. Xie, J. Zhu, X. Li, and Y. Li. Study of moiré grating fabrication on metal samples using nanoimprint lithography. Optics Express, 20:2942-2955, 2012.

[39] J.W. Dally and D.T. Read. Electron beam moiré. Experimental Mechanics, $59(4): 270-277,33$.

[40] D.Z. Read and J.W. Dally. Theory of electron beam moiré. Journal of Research of the National Institute of Standards and Technology, 101(1):47-61, 1996.

[41] H. Chen and D. Liu. Advances in scanning electron microscope moiré. Experimental Mechanics, 41:165-173, 2001.

[42] C. Luo, Y. Li, and S. Susumu. Fabrication of high aspect ratio subwavelength gratings based on x-ray lithography and electron beam lithography. Optics and Lasers Technology, 44(7):1649-53, 2012.

[43] H. Xie, B. Li, R. Geer, B. Xu, and J. Castracane. Focused ion beam moiré method. Optics and Lasers in Engineering, 40(3):163-177, 2003.

[44] L. Biao, X. Tang, X. Huimin, and Z. Xin. Focused ion beam (FIB) nano-machining and FIB moiré technique for strain analysis in MEMS/NEMS structures and devices. In Proceedings of the IEEE Sixteenth Annual International Conference on Micro Electro Mechanical Systems, pages 674-677, 2003.

[45] Y.J. Li, H.M. Xie, B.Q. Guo, Q. Luo, C.Z. Gu, and M.Q. Xu. Fabrication of highfrequency moiré gratings for microscopic deformation measurement using focused ion beam milling. Optics and Lasers in Engineering, 20(5):055037, 2010.

[46] J. Ming, T. Min-Jin, W. Hao, L. Yan-Jie, and X. Hui-Min. FIB moiré gratings and their application in the measurement of optical fibers' mechanical properties. Chinese Physics Letters, 29:034210, 2012.

[47] C. Pinna, J.H. Beynon, C.M. Sellars, and M. Bornert. Experimental investigation and micromechanical modeling of the hot deformation of duplex stainless steels. In Proceedings of the International Conference on Mathematical Modelling in Metal Processing and Manufacturing-COM 2000, Ottawa, Ontario, Canada, 2000. 
[48] C. Gérard, F. N'Guyen, N. Osipov, G. Cailletaud, M. Bornert, and D. Caldemaison. Comparison of experimental results and finite element simulation of strain localization scheme under cyclic loading. Computational Materials Science, 46(3):755-760, 2009.

[49] G. Martin, D. Caldemaison, M. Bornert, C. Pinna, Y. Bréchet, M. Véron, J.D. Mithieux, and T. Pardoen. Characterization of the high temperature strain partitioning in duplex steels. Experimental Mechanics, 53(2):205-215, 2013.

[50] M.L. Basehore and D. Post. High frequency, high reflectance transferable moiré gratings. Experimental Techniques, 8(5):29-31, 1984.

[51] M. B. Whitworth and J.M. Huntley. Dynamic stress analysis by high-resolution reflection moiré photography. Optical Engineering, 33(3):924-931, 94.

[52] B. Zhao, A. K. Asundi, and K. E. Oh. Grid method for strain measurement in electronic packaging using optical, electronic, and atomic force microscopy. In R. A. Lieberman, A. K. Asundi, and H. Asanuma, editors, Proc. SPIE 3897, Advanced Photonic Sensors and Applications, 260, 1999.

[53] B. Zhao and A. Asundi. Microscopic grid method - resolution and sensitivity. Optics and Lasers in Engineering, 36(5):437-450, 2001.

[54] H. Xie, A. Asundi, C. G. Boay, L. Yunguang, J. Yu, Z. Zhaowei, and B. K. A. Ngoi. High resolution AFM scanning moiré method and its application to the microdeformation in the BGA electronic package. Microelectonics Reliability, 42(8):12191227, 2002.

[55] C.A. Sciammarella, EM. Sciammarella, and T. Kim. Strain measurements in the nanometer range in a particulate composite using computer-aided moiré. Experimental Mechanics, 43(3):341-347, 2003.

[56] B. M. Schroeter and D.L. McDowell. Measurement of deformation fields in polycrystalline OFHC copper. International Journal of Plasticity, 19(9):1355-1376, 2003.

[57] R. Moulart, R. Rotinat, F. Pierron, and G. Lerondel. On the realization of microscopic grids for local strain measurement by direct interferometric photolithography. Optics and Lasers in Engineering, 45(12):1131-1147, 2007.

[58] D.T. Read, J.W. Dally, and M. Szanto. Scanning moiré at high magnification using optical methods. Experimental Mechanics, 33(2):110-116, 1993.

[59] D. Post, B. Han, and P. Ifju. High Sensitivity Moire-Experimental Analysis for Mechanics and Materials. Springer-Verlag, 1994. ISBN 978-1-4612-4334-2, 444 pages.

[60] P. Doumalin, M. Bornert, and J. Crepin. Caractérisation de la répartition de la déformation dans les matériaux hétérogènes / characterisation of the strain distribution in heterogeneous materials. Mécanique et Industries, 4:607-617, 2003.

[61] J. Molimard and Y. Surrel. Grid method, moiré and deflectometry. In M. Grédiac and F. Hild, editors, Full-field Measurements and Identification in Solid Mechanics, chapter 3, pages 61-89. Wiley, 2012. ISBN: 978-1-118-57847-6.

[62] A. R. Luxmoore and R. Herrnann. The rapid deposition of moiré grids. Experimental Mechanics, 11(5):375-377, 1971. 
[63] https://www.tedpella.com/grids_html/grids.htm.

[64] H. T. Goldrein, S. J. P. Palmer, and J. M. Huntley. Automated fine grid technique for measurement of large-strain deformation map. Optics and Lasers in Engineering, 23(5):305-318, 1995.

[65] S. Hedan, V. Valle, and M. Cottron. In-plane displacement formulation for finite cracked plates under mode I using grid method and finite element analysis. Experimental Mechanics, 50(3):401-412, 2010.

[66] X. Li, Z. Zhang, L. Qin, X. Feng b, Z. Feng, L. He, Y. Wang, H. Miao, and X. Gong. High strain gradient measurements using modified automated grid technique. Optics and Lasers in Engineering, 52:140-144, 2014.

[67] B. Ribbens. Development and validation of a time-domain fringe pattern analysis technique for the measurement of object shape and deformation. PhD thesis, Vrije Universiteit Brussel and Universiteit Antwerpen, 2015. ISBN 9789057183818.

[68] http://www.themagictouch.com/.

[69] S. Avril, A. Vautrin, and Y. Surrel. Grid method: Application to the characterization of cracks. Experimental Mechanics, 44(1):37-43, 2004.

[70] E. Toussaint, J. F. Destrebecq, and M. Grédiac. A refined study of the crack propagation in cement-based composite beams under bending. Cement and Concrete Composites, 27(2):399-411, 2005.

[71] S. Sun, M. Grédiac, E. Toussaint, J.M. Mathias, and N. Mati-Baouche. Applying a full-field measurement technique to characterize the mechanical response of a sunflower-based biocomposite. Experimental Mechanics, 55(5):917-934, 2015.

[72] Y. Morimoto, Y. Seguchi, and T. Higashi. Strain analysis by mismatch moiré method and grid method using Fourier transform. Computational Mechanics, 6:1-10, 1990.

[73] P.J. Rae, H.T. Goldrein, N.K. Bourne, W.G. Proud, L.C. Forde, and M. Liljekvist. Measurement of dynamic large-strain deformation maps using an automated grid technique. Optics and Lasers in Engineering, 31(2):113-122, 1999.

[74] S. Ri, M. Fujigaki, and Y. Morimoto. Sampling moiré method for accurate small deformation distribution measurement. Experimental Mechanics, 50(4):501-508, 2010.

[75] S. Ri, T. Muramatsu, M. Saka, K. Nanbara, and D. Kobayashi. Accuracy of the sampling moiré method and its application to deflection measurements of large-scale structures. Experimental Mechanics, 52(4):331-3340, 2012.

[76] S. Ri, T. Muramatsu, M. Saka, K. Nanbara, and D. Kobayashi. Noncontact deflection distribution measurement for large-scale structures by advanced image processing technique. Materials Transactions, 53(2):323-329, 2012.

[77] Z. W. Liu, J. F. Zhou, C. L. Wu, and H. M. Xie. GPA combined with the holedrilling method applied for curved surface strain measurement. Strain, 49(1):46-53, 2012 .

[78] M. Grédiac and F. Sur. Effect of sensor noise on the resolution and spatial resolution of the displacement and strain maps obtained with the grid method. Strain, 50(1):1$27,2014$. 
[79] https://www.appliedimage.com/products/sine-patterns-and-square-wave-targets.

[80] C. Badulescu, M. Grédiac, and J.-D. Mathias. Investigation of the grid method for accurate in-plane strain measurement. Measurement Science and Technology, 20(9):20:095102, 2009 .

[81] Y. Surrel. Moiré and grid methods: a signal-processing approach. In Stupnicki J. Pryputniewicz R.J., editor, Proceedings SPIE, Interferometry '94: Photomechanics, volume 2342, pages 118-127. SPIE, 1994.

[82] M. Takeda, H. Ina, and S. Kobayashi. Fourier-transform method of fringe-pattern analysis for computer-based topography and interferometry. Journal of the Optical Society of America, 72(1):156-160, 1982.

[83] M. Takeda and K. Mutoh. Fourier transform profilometry for the automatic measurement of 3-d object shapes. Applied Optics, 22(24):3977-3982, 1983.

[84] M. Takeda. Spatial-carrier fringe-pattern analysis and its applications to precision interferometry and profilometry: An overview. Industrial Metrology, 1(2):79-99, 1990 .

[85] M.A. Khamsi and W.A. Kirk. An introduction to metric spaces and fixed point theory. Wiley, 2001.

[86] J.M. Huntley. Noise-immune phase unwrapping algorithm. Applied Optics, 28(16):3268-3270, 1989.

[87] J.M. Huntley and H. Saldner. Temporal phase-unwrapping algorithm for automated interferogram analysis. Applied Optics, 32(17):3047-3052, 1993.

[88] M.A. Herraez, D.R. Burton, M.J. Lalor, and D.B. Clegg. Robust, simple, and fast algorithm for phase unwrapping. Applied Optics, 35(29):5847-5852, 1996.

[89] J.M. Huntley. Automated fringe pattern analysis in experimental mechanics: A review. The Journal of Strain Analysis for Engineering Design, 33(2):105-125, 1998.

[90] D.C. Ghiglia and M.D. Pritt. Two-dimensional phase unwrapping: theory, algorithm and software. Wiley, 1998.

[91] Z. Hongbin and W. Qing. Measuring instantaneous frequencies of fringe image and its application in phase unwrapping. Acta Electron Sinica, 27:15-8, 1999.

[92] M.A. Herraez, D.R. Burton, M.J. Lalor, and M.A. Gdeisat. Fast two-dimensional phase-unwrapping algorithm based on sorting by reliability following a noncontinuous path. Applied Optics, 41(35):7437-7444, 2002.

[93] M.A. Herraez, M.A. Gdeisat, D.R. Burton, and M.J. Lalor. Robust, fast, and effective two-dimensional automatic phase unwrapping algorithm based on image decomposition. Applied Optics, 41(35):7445-7455, 2002.

[94] M.A. Herraez, J.G. Boticario, M.J. Lalor, and D.R. Burton. Agglomerative clustering-based approach for two-dimensional phase unwrapping. Applied optics, 44(7):1129-1140, 2005. 
[95] E. Zappa and G. Busca. Comparison of eight unwrapping algorithms applied to Fourier-transform profilometry. Optics and Lasers in Engineering, 46(2):106-116, 2008.

[96] http://www.mathworks.com/matlabcentral/fileexchange/22504-2d-phaseunwrapping-algorithms.

[97] https://pypi.python.org/pypi/unwrap.

[98] https://www.ljmu.ac.uk/research/centres-and-institutes/faculty-of-engineeringand-technology-research-institute/geri/phase-unwrapping.

[99] C. Badulescu, M. Grédiac, J.-D. Mathias, and D. Roux. A procedure for accurate one-dimensional strain measurement using the grid method. Experimental Mechanics, 49(6):841-854, 2009.

[100] B. Blaysat, M. Grédiac, and F. Sur. Effect of interpolation on noise propagation from images to DIC displacement maps. International Journal for Numerical Methods in Engineering, 2016. DOI: 10.1002/nme.5212.

[101] B. Blaysat, M. Grédiac, and F. Sur. On the propagation of camera sensor noise to displacement maps obtained by DIC - An experimental study. Experimental Mechanics, 2016. DOI:10.1007/s11340-016-0130-9.

[102] S. Avril, P. Feissel, F. Pierron, and P. Villon. Estimation of the strain field from full-field displacement noisy data. Comparing finite elements global least squares and polynomial diffuse approximation. European Journal of Computational Mechanics, $17(5-7): 857-868,2008$.

[103] S. Avril, P. Feissel, F. Pierron, and P. Villon. Comparison of two approaches for differentiating full-field data in solid mechanics. Measurement Science and Technology, 21(1):015703, 2010.

[104] P. Feissel. From displacement to strain. In M. Grédiac and F. Hild, editors, Fullfield Measurements and Identification in Solid Mechanics, chapter 7, pages 191-222. Wiley, 2012. ISBN: 978-1-118-57847-6.

[105] X. Su and W. Chen. Fourier transform profilometry: a review. Optics and Lasers in Engineering, 35(5):263-284, 2001.

[106] M. Takeda. Fourier fringe analysis and its application to metrology of extreme physical phenomena: a review. Applied Optics, 52(1):20-29, 2013.

[107] Q. Kemao. Windowed Fourier transform for fringe pattern analysis. Applied Optics, 43(13):2695-2702, 2004.

[108] Q. Kemao. Two-dimensional windowed Fourier transform for fringe pattern analysis: Principles, applications and implementations. Optics and Lasers in Engineering, 45(2):304-317, 2007.

[109] Q. Kemao. Applications of windowed Fourier fringe analysis in optical measurement: a review. Optics and Lasers in Engineering, 66(2):67-73, 2015.

[110] H. Liu, A.N. Cartwright, and C. Basaran. Moiré interferogram phase extraction: a ridge detection algorithm for continuous wavelet transforms. Applied Optics, 43(4):850-857, 2004. 
[111] J. Zhong and J. Weng. Spatial carrier-fringe pattern analysis by means of wavelet transform: Wavelet transform profilometry. Applied Optics, 43:4993-4998, 2004.

[112] J. Zhong and J. Weng. Phase retrieval of optical fringe patterns from the ridge of a wavelet transform. Optics Letters, 30:2560-2562, 2005.

[113] Y. Morimoto, Z. Hayashi, and K. Wada. High-sensitivity measurement of strain by moiré interferometry. Transactions of the Japan Society for Mechanical Engineers Series A, 31(1):122-127, 1987.

[114] Y. Morimoto, Y. Seguchi, and T. Higashi. Two-dimensional moiré method and grid method using Fourier transform. Experimental Mechanics, 4:399-404, 1989.

[115] F. Brémand, J.-C. Dupré, and A. Lagarde. Non-contact non-disturbing local strain measurement method. I- principle. European Journal of Mechanics A/Solids, 11(3):349-366, 1992.

[116] M. Cottron, F. Brémand, and A. Lagarde. Non-contact and non-disturbing local strain measurement methods. 2- applications. European Journal of Mechanics, A/Solids, 11(3):367-379, 1992.

[117] J.C. Dupré, F. Brémand, and A. Lagarde. Numerical spectral analysis of a grid: application to strain measurement. Optics and Lasers in Engineering, 18(3):159$172,1993$.

[118] P. Sevenhuijsen, J. Sirkis, and F. Brémand. Current trends in obtaining deformation data from grids. Experimental Techniques, 17(3):22-26, 1993.

[119] X. Dai, H. Xie, H. Wang, C. Li, Z. Liu, and L. Wu. The geometric phase analysis method based on the local high resolution discrete Fourier transform for deformation measurement. Measurement Science and Technology, 25(2):025402, 2014.

[120] M. J. Hÿtch and L. Potez. Geometric phase analysis of high-resolution electron microscopy images of antiphase domains: example Cu3Au. Philosophical Magazine A, 74:1119-1138, 1997.

[121] Y. Surrel. Design of algorithms for phase measurements by the use of phase stepping. Applied Optics, 35(1):51-60, 1996.

[122] X. Dai, H. Xie, and H. Wang. Geometric phase analysis based on the windowed Fourier transform for the deformation field measurement. Optics and Laser Technology, 58(6):119-127, 2014.

[123] E. Robin, V. Valle, and F. Brémand. Phase demodulation method from a single fringe pattern based on correlation with a polynomial form. Applied Optics, 44(34):7261-7269, 2005.

[124] S. Ri and T. Muramatsu. Theoretical error analysis of the sampling moiré method and phase compensation methodology for single-shot phase analysis. Applied Optics, 51(16):3214-3223, 2012.

[125] C. Badulescu, M. Bornert, J.-C. Dupré, S. Equis, M. Grédiac, J. Molimard, P. Picart, R. Rotinat, and V. Valle. Demodulation of spatial carrier images: Performance analysis of several algorithms. Experimental Mechanics, 53(8):1357-1370, 2013. 
[126] L. Huang, Q. Kemao, B. Pan, and A.K. Asundi. Comparison of Fourier transform, windowed Fourier transform, and wavelet transform methods for phase extraction from a single fringe pattern in fringe projection profilometry. Optics and Lasers in Engineering, 48:141-148, 2010.

[127] M. K. Kujawinska and J. W. Wojciak. Spatial-carrier phase-shifting technique of fringe pattern analysis. In P. J. Juptner, editor, Industrial applications of holographic and seckle measuring techniques, volume SPIE 1508, pages 61-67, 1991.

[128] Y. Surrel. Phase-stepping. a new self-calibration algorithm. Applied Optics, 32(19):3598-3600, 1993.

[129] M. Pirga and M. Kujawinska. Two-directional spatial-carrier phase-shifting method for analysis of complex interferograms. In Interferometry 94: New techniques and analysis in optical measurements, volume SPIE 2340, pages 163-169, 1994.

[130] Y. Surrel. Additive noise effect in digital phase detection. Applied Optics, 36(1):271276, 1997.

[131] Y. Surrel. Design of phase-detection algorithms insensitive to bias modulation. Applied Optics, 36(4):805-807, 1997.

[132] Y. Surrel. Phase-shifting algorithms for nonlinear and spatially nonuniform phase shifts: comment. Journal of the optical Society of America A, 15(5):1227-1233, 1998.

[133] Y. Surrel. Extended averaging and data windowing techniques in phase-stepping measurements: an approach using the characteristic polynomial theory. Optical Engineering, 37(8):2314-2319, 1998.

[134] B. Zhao and Y. Surrel. Phase-shifting sample self-calibration algorithm insensitive to the 2nd-harmonics in the fringe signal. Optical Engineering, 34(9):2821-2822, 1995.

[135] B. Zhao and Y. Surrel. Effect of quantization error on the computed phase of phaseshifting measurements. Applied Optics, 36(12):2070-2075, 1997.

[136] J. Xu, Q. Xu, and H. Peng. Spatial carrier phase-shifting algorithm based on leastsquares iteration. Applied Optics, 47(29):5446-5453, 2008.

[137] F. Sur and M. Grédiac. Influence of the analysis window on the metrological performance of the grid method. Journal of Mathematical Imaging and Vision, 2016. DOI: $10.1007 / \mathrm{s} 10851-016-0650-\mathrm{z}$.

[138] S. Mallat. A Wavelet Tour of Signal Processing. Academic Press, third edition, 2008.

[139] E. A. Patterson, E. Hack, P. Brailly, R. L. Burguete, Q. Saleeme, T. Siebert, R. A. Tomlinsone, and M. P. Whelan. Calibration and evaluation of optical systems for full-field strain measurement. Optics and Lasers in Engineering, 45(5):550-564, 2007.

[140] E. Hack, G. Lampeas, J.E. Mottershead, E.A. Patterson, T. Siebert, and M.P. Whelan. Progress in developing a standard for dynamic strain analysis. In Experimental and Applied Mechanics, volume 6 of Conference Proceedings of the Society for Experimental Mechanics Series, pages 425-429, 2011. 
[141] A. Davighi, R.L. Burguete, M. Feligiotti, E. Hack, S. James, E.A. Patterson, T. Siebert, and M.P. Whelan. he development of a reference material for calibration of full-field optical measurement systems for dynamic deformation measurements. Applied Mechanics and Materials, 70:33-38, 2011.

[142] E. Hack, X. Lin, E. A. Patterson, and C. M. Sebastian. A reference material for establishing uncertainties in full-field displacement measurements. Measurement Science and Technology, 26(7):075004, 2015.

[143] D.Wang, F.A. DiazDelaO, W. Wang, X. Lin, E. A. Patterson, and J. E. Mottershead. Uncertainty quantification in DIC with kriging regression. Optics and Lasers in Engineering, 78:182 - 195, 2016.

[144] http://cordis.europa.eu/result/brief/rcn/3262_en.html.

[145] http://www.dynamicvalidation.org/.

[146] http://www.engineeringvalidation.org/.

[147] Standard guide for evaluating non-contacting optical strain measurement systems, ASTM standard E2208-02, 2010.

[148] T. Siebert and E. Hack. Draft standard guide for optical deformation measurements in dynamic events. 2011. Deliverable D2.8 of the ADVISE European project.

[149] S. Roux and F. Hild. Stress intensity factor measurements from digital image correlation: post-processing and integrated approaches. International Journal of Fracture, 140(1-4):141-157, 2006.

[150] Z. Y. Wang, H. Q. Li, J. W. Tonga, and J. T. Ruan. Statistical analysis of the effect of intensity pattern noise on the displacement measurement precision of digital image correlation using self-correlated images. Experimental Mechanics, 47(5):701-707, 2007.

[151] J. Réthoré, G. Besnard, G. Vivier, F. Hild, and S. Roux. Experimental investigation of localized phenomena using digital image correlation. Philosophical Magazine, 88(28-29):3339-3355, 2008.

[152] H. Haddadi and S. Belhabib. Use of a rigid-body motion for the investigation and estimation of the measurement errors related to digital image correlation technique. Optics and Lasers in Engineering, 46(2):185-96, 2008.

[153] P. Lava, S. Cooreman, S. Coppieters, M. DeStrycker, and D. Debruyne. Assessment of measuring errors in DIC using deformation fields generated by plastic FEA. Optics and Lasers in Engineering, 47(7):747-53, 2009.

[154] Y. Q. Wang, M. A. Sutton, H. A. Bruck, and H. W. Schreier. Quantitative error assessment in pattern matching: effects of intensity pattern noise, interpolation, strain and image contrast on motion measurements. Strain, 45(2):160-78, 2009.

[155] J. Réthoré. A fully integrated noise robust strategy for the identification of constitutive laws from digital images. Internation Journal for Numerical Methods in Engineering, 84(6):631-660, 2010. 
[156] Y. Q. Wang, M. A. Sutton, X. D. Ke, H.W. Schreier, P. L. Reu, and T. J. Miller. On error assessment in stereo-based deformation measurements. Part 1: Theoretical developments for quantitative estimates. Experimental Mechanics, 51(4):405-422, 2011.

[157] X. D. Ke, H. W. Schreier, M. A. Sutton, and Y. Q. Wang. Error assessment in stereobased deformation measurements. Part 2: Experimental validation of uncertainty and bias estimates. Experimental Mechanics, 51(4):423-441, 2011.

[158] F. Hild and S. Roux. Comparison of local and global approaches to digital image correlation. Experimental Mechanics, 52(9):1503-1519, 2012.

[159] JCGM Member Organizations. International vocabulary of metrology - Basic and general concepts and associated terms (VIM). BIPM, 2008.

[160] ISO 5725. Accuracy (trueness and precision) of measurement methods and results, 1994. The International Organization for Standardization.

[161] P. Lava, W. Van Paepegem, S. Coppieters, I. De Baere, and Y. Wang. Impact of lens distortions on strain measurements obtained with 2D digital image correlation. Optics and Lasers in Engineering, 51(5):576-584, 2013.

[162] B. Pan, L. Yu, and D. Wu. High-accuracy 2D digital image correlation measurements with bilateral telecentric lenses: Error analysis and experimental verification. Experimental Mechanics, 53(9):1719-1733, 2013.

[163] L. Wittevrongel, M. Badaloni, R. Balcaen, P. Lava, and D. Debruyne. Evaluation of methodologies for compensation of out of plane motions in a $2 \mathrm{D}$ digital image correlation setup. Strain, 51(5):357-369, 2015.

[164] A. Chrysochoos and Y. Surrel. Basics of metrology and introduction to techniques. In M. Grédiac and F. Hild, editors, Full-field Measurements and Identification in Solid Mechanics, chapter 1, pages 1-29. Wiley, 2012. ISBN: 978-1-118-57847-6.

[165] M. Bornert, F. Brémand, P. Doumalin, J.-C. Dupré, M. Fazzini, M. Grédiac, F. Hild, S. Mistou, J. Molimard, J.-J. Orteu, L. Robert, Y. Surrel, P. Vacher, and B. Wattrisse. Assessment of digital image correlation measurement errors: methodology and results. Experimental Mechanics, 49(3):353-370, 2009.

[166] DIC challenge: http://sem.org/dic-challenge/.

[167] L. Wittevrongel, P. Lava, S. V. Lomov, and D. Debruyne. A self adaptive global digital image correlation algorithm. Experimental Mechanics, 55(2):361-378, 2015.

[168] J. Blaber, B. Adair, and A. Antoniou. Ncorr: Open-source 2D digital image correlation Matlab software. Experimental Mechanics, 55(6):1105-1122, 2015.

[169] M. Grédiac, F. Sur, C. Badulescu, and J.-D. Mathias. Using deconvolution to improve the metrological performance of the grid method. Optics and Lasers in Engineering, 51(6):716-734, 2013.

[170] E. W. Grafarend. Linear and Nonlinear Models: Fixed Effects, Random Effects, and Mixed Models. Walter de Gruyter, 2006. ISBN-13:978-3-11-016216-5, 752 pages. 
[171] F. Sur and M. Grédiac. Towards deconvolution to enhance the grid method for in-plane strain measurement. Inverse Problems and Imaging, 8(1):259-291, 2014.

[172] J. Molimard and L. Navarro. Uncertainty on fringe projection technique: a MonteCarlo-based approach. Optics and Lasers in Engineering, 51(7):840-847, 2013.

[173] J. Hadamard. Sur les problèmes aux dérivées partielles et leur signification physique. Princeton University Bulletin, 13:49-52, 1902.

[174] R.C. Gonzalez and R. E. Wood. Digital Image Processing. Pearson Education, 2009.

[175] J.L. Starck, E. Pantin, and F. Murtagh. Deconvolution in astronomy: a review. Publications of the astronomical society of the pacific, 114(800):1051-1069, 2002.

[176] F. Sur, B. Blaysat, and M. Grédiac. Determining displacement and strain maps immune from aliasing effect with the grid method. 2016. Submitted.

[177] M. Berge-Gras and J. Molimard. Optimisation de la dérivation en fonction de la résolution et de la résolution spatiale sur les déformations. In Proceedings of the 19th French National Conference on Mechanics, Marseille, pages 1-6, 2009. in French.

[178] S.D. Forman, J.D. Cohen, M. Fitzgerald, W.F. Eddy, M.A. Mintun, and D.C. Noll. Improved assessment of significant activation in functional magnetic resonance imaging (fMRI): Use of a cluster-size threshold. Magnetic Resonance in Medicine, 33(5):636-647, 1995.

[179] M. Brett, W. Penny, and S. Kiebel. Introduction to random field theory. In J. Ashburner, K. Friston, and W. Penny, editors, Human brain function, Part II. Elsevier, 2nd edition edition, 2003.

[180] K.J. Worsley. Developments in random field theory. In J. Ashburner, K. Friston, and W. Penny, editors, Human brain function, Part II. Elsevier, 2nd edition edition, 2003.

[181] G.-Q. Wei and S. Ma. Implicit and explicit camera calibration: theory and experiments. IEEE Transactions on Pattern Analysis and Machine Intelligence, 16(5):469480, 1994.

[182] S. Coudert, K. Triconnet K., A. Zebiri, and Y. Surrel. Étalonnage transverse d'un objectif de caméra par la méthode de la grille. In Proceedings of Photomécanique 2004, pages 257-264, 2004.

[183] Z. Zhang. A flexible new technique for camera calibration. Technical Report MSRTR-98-71, Microsoft Research, 1998.

[184] J. Heikkila and O. Silven. A four-step camera calibration procedure with implicit image correction. In Proceedings of the International Conference on Computer Vision and Pattern Recognition (CVPR), pages 1106-1112, 1997.

[185] G.E. Healey and R. Kondepudy. Radiometric ccd camera calibration and noise estimation. IEEE Transactions on Pattern Analysis and Machine Intelligence, 16(3):267-276, 1994.

[186] J. Boulanger, C. Kervrann, P. Bouthemy, P. Elbau, J.-B. Sibarita, and J. Salamero. Patch-based nonlocal functional for denoising fluorescence microscopy image sequences. IEEE Transaction on Medical Imaging, 29(2):442-454, 2010. 
[187] H. Faraji and W.J. MacLean. CCD noise removal in digital images. IEEE Transactions on Image Processing, 15(9):2676-2685, 2006.

[188] A. Foi, M. Trimeche, V. Katkovnik, and K. Egiazarian. Practical PoissonianGaussian noise modeling and fitting for single-image raw-data. IEEE Transactions on Image Processing, 17(10):1737-1754, 2008.

[189] F.J. Anscombe. The transformation of Poisson, binomial and negative-binomial data. Biometrika, 35(3-4):246-254, 1948.

[190] F. Murthagh, J.L. Starck, and A. Bijaoui. Image restoration with noise suppression using a multiresolution support. Astronomy and astrophysics, 112:179-189, 1995.

[191] F. Sur and M. Grédiac. Sensor noise modeling by stacking pseudo-periodic grid images affected by vibrations. IEEE Signal Processing Letters, 21(4):432-436, 2014.

[192] F. Sur and M. Grédiac. On noise reduction in strain maps obtained with the grid method by averaging images affected by vibrations. Optics and Lasers in Engineering, 66:210-222, 2015.

[193] D.P. Petersen and D. Middleton. Sampling and reconstruction of wave-numberlimited functions in $n$-dimensional Euclidean spaces. Information and Control, 5:279-323, 1962 .

[194] M. Grédiac, F. Sur, and B. Blaysat. Removing quasi-periodic noise in strain maps by filtering in the Fourier domain. Experimental Techniques, 2015. DOI: 10.1111/ext.12162.

[195] F. Sur and M. Grédiac. Automated removal of quasiperiodic noise using frequency domain statistics. ISETT/SPIE Journal of Electronic Imaging, 24(1):013003/1-19, 2015.

[196] S. Ri, M. Saka, K. Nanbara, and D. Kobayashi. Dynamic thermal deformation measurement of large-scale high-temperature piping in thermal power plants utilizing the sampling moiré method and grating magnets. Experimental Mechanics, 53(9):1635-1646, 2013.

[197] Y. Surrel, N. Fournier, M. Grédiac, and P.-A. Paris. Phase-stepped deflectometry applied to shape measurement of bent plates. Experimental Mechanics, 39(1):66-70, 1999.

[198] J.H. Kim, F. Pierron, M. Grédiac, and M. R. Wisnom. A procedure for producing reflective coatings on plates to be used for full-field slope measurements by a deflectometry technique. Strain, 43(2):138-143, 2007.

[199] M. Grédiac, N. Fournier, P.-A. Paris, and Y. Surrel. Direct identification of elastic constants of anisotropic plates by modal analysis : experiments and results. Journal of Sound and Vibration, 210(5):645-659, 1998.

[200] M. Grédiac, N. Fournier, P.-A. Paris, and Y. Surrel. Direct measurement of invariant parameters of composite plates. Journal of Composite Materials, 33(20):1939-1965, 1999. 
[201] C. Devivier, F. Pierron, and M.R. Wisnom. Impact damage detection in composite plates using deflectometry and the virtual fields method. Composites Part A: Applied Science and Manufacturing, 48:201-218, 2013.

[202] J. Xavier, U. Belini, F. Pierron, F. Morais, J. Lousada, and M. Tomazello. Characterisation of the bending stiffness components of mdf panels from full-field slope measurements. Wood Science and Technology, 47(2):423-441, 2013.

[203] R. Moutou Pitti, C. Badulescu, and M. Grédiac. Characterization of a cracked specimen with full-field measurements: direct determination of the crack tip and energy release rate calculation. International Journal of Fracture, 187(1):109-121, 2014 .

[204] S. Avril, A. Vautrin, and Y. Surrel. Grid method: application to the characterization of cracks. Experimental Mechanics, 44(1):37-43, 2004.

[205] E. Toussaint, E. Fournely, R. Moutou Pitti, and M. Grédiac. Studying the mechanical behavior of notched beams with full-field measurements. Engineering Structures, 113(5):277-286, 2016.

[206] D. Delpueyo, M. Grédiac, X. Balandraud, and C. Badulescu. Investigation of martensitic microstructures in a monocrystalline $\mathrm{Cu}-\mathrm{Al}$-Be shape memory alloy with the grid method and infrared thermography. Mechanics of Materials, 45(1):34-51, 2012.

[207] C. Badulescu, M. Grédiac, H. Haddadi, J.-D. Mathias, X. Balandraud, and H.-S. Tran. Applying the grid method and infrared thermography to investigate plastic deformation in aluminium multicrystal. Mechanics of Materials, 43(11):36-53, 2011.

[208] X. Balandraud, N. Barrera, P. Biscari, M. Grédiac, and G. Zanzotto. Strain intermittency in shape memory alloys. Physical Review B, 91(17):174111, 2015.

[209] M. Grédiac and E. Toussaint. Studying the mechanical behaviour of asphalt mixtures with the grid method. Strain, 49(1):1-15, 2013.

[210] M. Grédiac, E. Toussaint, C. Petit, A. Millien, and D. C. Nguyen. A comparative study of the heterogeneous local mechanical response of two types of asphalt mixes. Materials and Structures, 47(9):1513-1529, 2014.

[211] M. C. Teguedi, B. Blaysat, E. Toussaint, S. Moreira, S. Liandrat, and M. Grédiac. Studying the influence of reclaimed asphalt pavement on local deformation properties of mixtures. 2016. Submitted.

[212] D. Dang, E. Toussaint, R. Moutou-Pitti, and M. Grédiac. Investigation of hydric transfer phenomenon in wood at the ring scale with the grid method. In SEM annual conference "Experimental Mechanics Spanning Multiple Scientific and Engineering Disciplines", Costa Mesa, USA, 2015.

[213] P. L. Reu, E. Quintana, and K. Lon. Using sampling moiré to extract displacement information from X-ray images of molten salt batteries. In Springer, editor, Proceedings of the 2014 Annual Conference on Experimental and Applied Mechanics, volume 3, "advancement of Optical Methods in Experimental Mechanics" of Conference proceedings of the Society for Experimental Mechanics series, pages 331-336, 2014. 
[214] M. J. Hÿtch, J-L. Putaux, and J-M. Pénisson. Measurement of the displacement field around dislocations to 0.03 angströms by electron microscopy. Nature, 423:270-273, 2003.

[215] M.J. Hÿtch, J.-L. Putaux, and J. Thibault. Stress and strain around grain-boundary dislocations measured by high-resolution electron microscopy. Philosophical Magazine, 86:4641-4656, 2006.

[216] C. W. Zhao, Y.M. Xing, C.E. Zhou, and P.C Bai. Experimental examination of displacement and strain fields in an edge dislocation core. Acta Materialia, 56(11):2570$2575,2008$.

[217] C.L. Johnson, E. Snoeck, M. Ezcurdia, B. Rodríguez-González, I. Pastoriza-Santos, L.M. Liz-Marzán, and M.J. Hÿtch. Effects of elastic anisotropy on strain distributions in decahedral gold nanoparticles. Nature Materials, 7:120-124, 2008.

[218] Z. Liu, H. Xie, D. Fang, F. Dai, Q. Xue, H. Liu, and J. Jia. Residual strain around a step edge of artificial nanocluster. Applied Physics Letters, 87(4):201908, 2004.

[219] C.W. Zhao and Y.M. Xing. Nanoscale deformation analysis of a crack-tip in silicon by geometric phase analysis and numerical moiré method. Optics and Lasers in Engineering, 48(11):1104 - 1107, 2010.

[220] J.J. Li, C.W. Zhao, Y.M. Xing, X.H. Hou, Z.C. Fan, Y.J. Jin, and Y. Wang. In-situ SEM investigation of sub-microscale deformation fields around a crack-tip in silicon. Optics and Lasers in Engineering, 50(12):1694 - 1698, 2012.

[221] C.W. Zhao, Y.M. Xing, P.C. Bai, J.F. Hou, and X.J. Dai. Quantitative measurement of deformation field around low-angle grain boundaries by electron microscopy. Physica B: Condensed Matter, 403(10-11):1838 -1842, 2008.

[222] F. Hüe, M.J. Hÿtch, H. Bender, F. Houdellier, and A. Claverie. Direct mapping of strain in a strained silicon transistor by highresolution electron microscopy. Physical Review Letters, 100:156602, 2008.

[223] J.L. Taraci, M.J. Hÿtch, T. Clement, P. Peralta, M.R. McCartney, J. Drucker, and S.T. Picraux. Strain mapping in nanowires. Nanotechnology, 403:2365-2371, 2005.

[224] Z.W. Liu, H.M. Xie, C.Z. Gu, and Y.G. Meng. The digital geometric phase technique applied to the deformation evaluation of MEMS devices. Journal of Micromechanics, 19(1):015012, 2009.

[225] E. Toussaint, A. Bouchair, M. Grediac, and S. Durif. Characterization of cover-plate bolted steel joints with full-field measurements. In SEM annual conference "Experimental Mechanics Spanning Multiple Scientific and Engineering Disciplines", Costa Mesa, USA, volume 3, 2015. Proceedings of the conference.

[226] S. Avril, M. Bonnet, A.S. Bretelle, M. Grédiac, F. Hild, P. Ienny, F. Latourte, D. Lemosse, S. Pagano, S. Pagnacco, and F. Pierron. Overview of identification methods of mechanical parameters based on full-field measurements. Experimental Mechanics, 48(4):381-402, 2008.

[227] J. Xavier, S. Avril, F. Pierron, and J. Morais. Novel experimental approach for longitudinal-radial stiffness characterisation of clear wood by a single test. Holzforschung, 61(5):573-581, 2007. 
[228] J. Xavier, S. Avril, F. Pierron, and J. Morais. Variation of transverse and shear stiffness properties of wood in a tree. Composites Part A: Applied Science and Manufacturing, 40(12):1953 -1960, 2009.

[229] M. Grédiac, F. Pierron, and Y. Surrel. Novel procedure for complete in-plane composite characterization using a single T-shaped specimen. Experimental Mechanics, 39(2):142-149, 1999.

[230] R. Moulart, S. Avril, and F. Pierron. Identification of the through-thickness rigidities of a thick laminated composite tube. Composites Part A: Applied Science and Manufacturing, 37(2):326 - 336, 2006.

[231] H. Chalal, S. Avril, F. Pierron, and F. Meraghni. Experimental identification of a nonlinear model for composites using the grid technique coupled to the virtual fields method. Composites Part A: Applied Science and Manufacturing, 37(2):315 - 325, 2006.

[232] F. Pierron, B. Green, M.R. Wisnom, and S.R. Hallett. Full-field assessment of the damage process of laminated composite open-hole tensile specimens. part 2: Experimental results. Composites Part A: Applied Science and Manufacturing, 38(11):2321 $-2332,2007$.

[233] Y. Pannier, S. Avril, R. Rotinat, and F. Pierron. Identification of elasto-plastic constitutive parameters from statically undetermined tests using the virtual fields method. Experimental Mechanics, 46(6):735-755, 2006.

[234] F. Pierron, S. Avril, and V. The Tran. Extension of the virtual fields method to elasto-plastic material identification with cyclic loads and kinematic hardening. International Journal of Solids and Structures, 47(22-23):2993-3010, 2010.

[235] R. Moulart, R. Rotinat, and F. Pierron. Full-field evaluation of the onset of microplasticity in a steel specimen. Mechanics of Materials, 41(11):1207 - 1222, 2009.

[236] M. Rossi, F. Pierron, and P. Forquin. Assessment of the metrological performance of an in situ storage image sensor ultra-high speed camera for full-field deformation measurements. Measurement Science and Technology, 25(2):025401, 2014.

[237] R. Moulart, F. Pierron, S.R. Hallett, and M.R. Wisnom. Full-field strain measurement and identification of composites moduli at high strain rate with the virtual fields method. Experimental Mechanics, 51(4):509-536, 2011.

[238] F. Pierron and P. Forquin. Ultra high-speed full-field deformation measurements on concrete spalling specimens and stiffness identification with the virtual fields method. Strain, 28(5):388-405, 2012.

[239] F. Pierron, H. Zhu, and C. Siviour. Beyond Hopkinson's bar. Philosophical Transactions of the Royal Society A: Mathematical, Physical and Engineering Sciences, 372(2023):20130195, 2014.

[240] G. Le Louedec, F. Pierron, M.A. Sutton, C. Siviour, and A.P. Reynolds. Identification of the dynamic properties of Al 5456 FSW welds using the virtual fields method. Journal of Dynamic Behavior of Materials, 2015. 


\section{List of Figures}

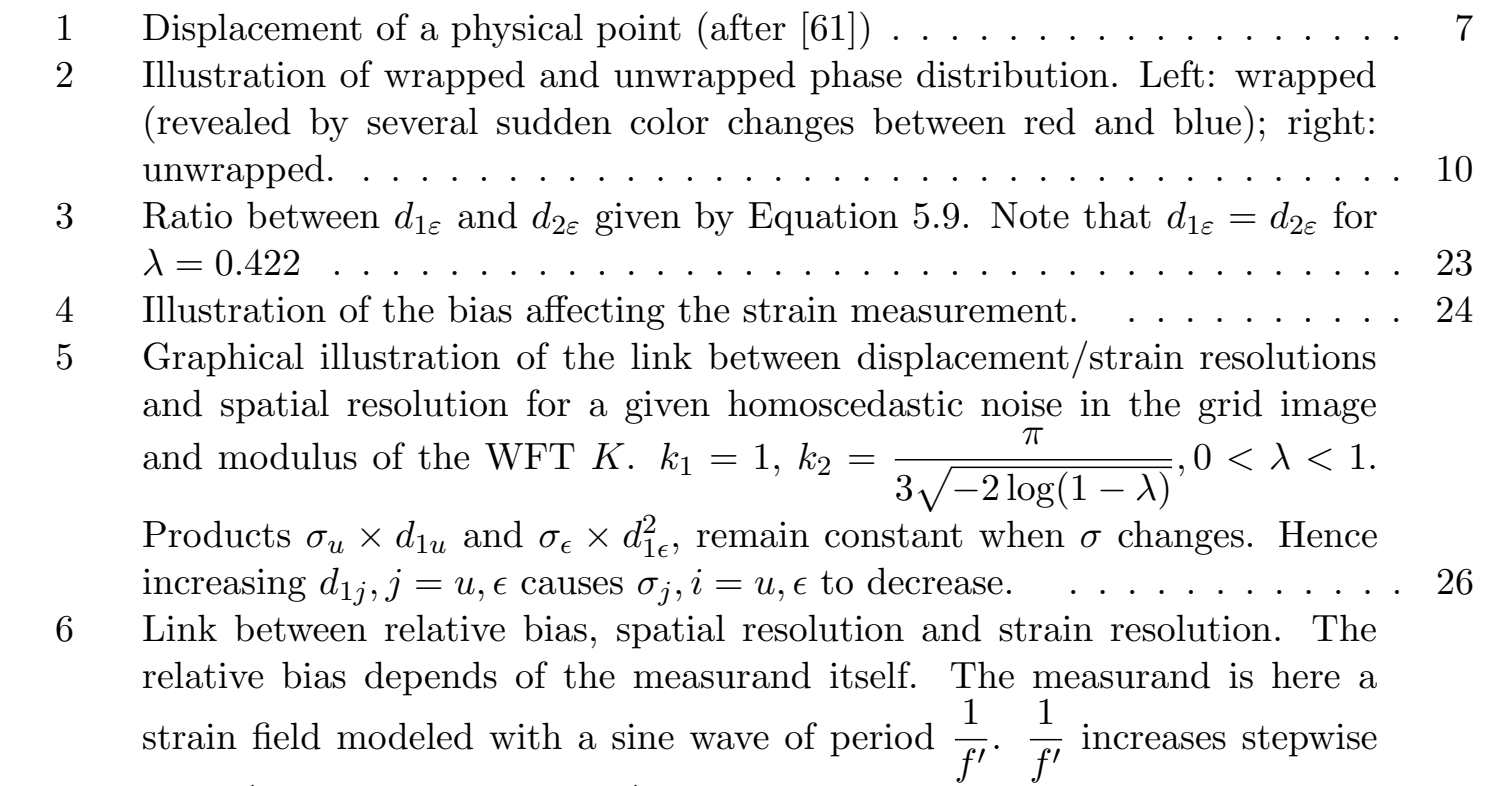

from $\frac{1}{f^{\prime}}=2 p=10$ pixels to $\frac{1}{f^{\prime}}=15 p=75$ pixels (step equal to $p$ ). $\ldots$. . . 29 29

7 Link between spatial resolution $d_{2 \epsilon}$, strain resolution $\sigma_{\epsilon}$ and relative bias $(\lambda)$. The bias $\lambda$ is in this case a parameter chosen by the user. It lies here

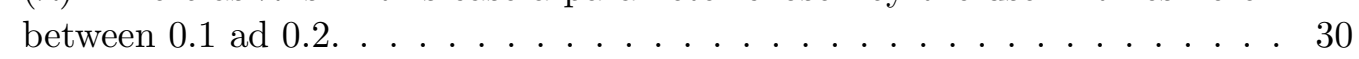

8 Cross-section along the $u$-axis of the weighting function affecting the Fourier

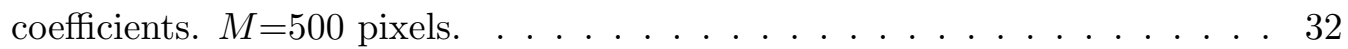

9 Typical experimental setup. A: camera, B: lighting, C: direct current transformer, D: digital camera, E: two translation stages, F: three-way

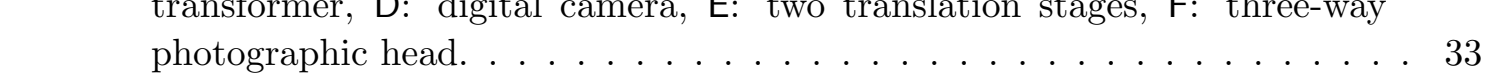

10 Close-ups of typical examples of blob distributions in experimental displacement and strain maps measured during a translation test. a- blobs in typical displacement maps are isotropic on average (here $u_{x}$ as an example). They are within a circle of diameter $6 \sqrt{2} \sigma$. b- blobs in $\epsilon_{x y}$ shear strain maps are isotropic on average. Their average dimension is smaller than those found for the displacement map (to be compared with the size of the blobs in subfigure a-). c- blobs in $\epsilon_{x x}$ strain maps are shrunk along direction $x$ dblobs in $\epsilon_{y y}$ strain maps are shrunk along direction $y$. e- correlation function for the random noise process in $\epsilon_{x x}$. f- correlation function for the random noise process in $\epsilon_{y y}$. All dimensions in pixels, 1 pixel represents $40 \mu \mathrm{m}$ on the specimen for subfigures a-, b-, c-, d-. $\ldots \ldots \ldots \ldots \ldots$

11 Illustrative example of the impact of grid defects on the displacement and strain maps, and defect removal by displacement compensation. a- vertical displacement $u_{y}$ (in micrometer). b- $\frac{\partial u_{y}}{\partial y}$ distribution. c- $\frac{\partial \Phi_{y}}{\partial y}$ distribution. Left: in the reference configuration. Right: in the current configuration. A slight vertical movement is visible between the two. d- $\varepsilon_{y y}$ with displacement compensation. dimensions in pixels, 1 pixel represents $40 \mu \mathrm{m}$ on the

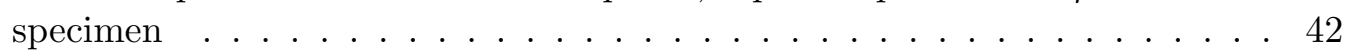

12 Removing parasitic fringes by filtering in the frequency domain, after [194]. 44 
13 Strain field near a crack tip in aluminium specimen (see [203]). dimensions in pixels, 1 pixel represents $40 \mu \mathrm{m}$ on the specimen. a- shear strain field. b- close-up of zone A. c- close-up of zone B . . . . . . . . . . . . . . . 46

14 Transverse strain field $\varepsilon_{y y}$ near the notch of a cracked notched wood beam specimen (see [205]). a- Schematic view of the notched beam. b- Strain field near the notch. dimensions in pixels, 1 pixel represents $20 \mu \mathrm{m}$ on the

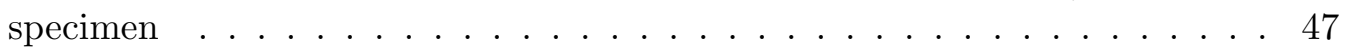

15 Tensile test on a monocrystal of shape memory alloy (see [206]). a- and btypical $\varepsilon_{x x}$ strain fields for different values of the load. c- typical deformed specimen during the test. dimensions in pixels, 1 pixel represents $40 \mu \mathrm{m}$ on

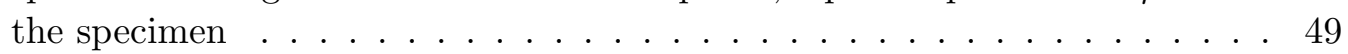

16 Asphalt specimen under vertical compression. a- picture of the front face before applying the grid. b- $\varepsilon_{y y}$ strain fields for a given value of the load (see [209]). The correspondence between the two sub-figures is clearly visible. c- deformed specimen. Dimensions in pixels, 1 pixel represents $40 \mu \mathrm{m}$ on

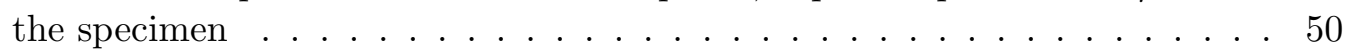

